\title{
Conformal geometry and (super)conformal higher-spin gauge theories
}

\section{Sergei M. Kuzenko and Michael Ponds}

Department of Physics M013, The University of Western Australia, 35 Stirling Highway, Crawley W.A. 6009, Australia

E-mail: sergei.kuzenko@uwa.edu.au, michael.ponds@research.uwa.edu.au

ABSTRACT: We develop a manifestly conformal approach to describe linearised (super)conformal higher-spin gauge theories in arbitrary conformally flat backgrounds in three and four spacetime dimensions. Closed-form expressions in terms of gauge prepotentials are given for gauge-invariant higher-spin (super) Cotton and (super) Weyl tensors in three and four dimensions, respectively. The higher-spin (super) Weyl tensors are shown to be conformal primary (super)fields in arbitrary conformal (super)gravity backgrounds, however they are gauge invariant only if the background (super) Weyl tensor vanishes. The proposed higher-spin actions are (super) Weyl-invariant on arbitrary curved backgrounds, however the appropriate higher-spin gauge invariance holds only in the conformally flat case. We also describe conformal models for generalised gauge fields that are used to describe partially massless dynamics in three and four dimensions. In particular, generalised higher-spin Cotton and Weyl tensors are introduced.

Keywords: Higher Spin Gravity, Higher Spin Symmetry, Supergravity Models, Superspaces

ARXIV EPRINT: 1902.08010 


\section{Contents}

1 Introduction 1

2 Conformal geometry 5

2.1 Gauging the conformal algebra 5

$\begin{array}{lll}2.2 & \text { Conformal gravity } & 7\end{array}$

$\begin{array}{lll}2.3 & \text { Conformal gravity in three dimensions } & 10\end{array}$

3 Conformal higher-spin models in three dimensions $\quad 13$

$\begin{array}{lll}3.1 & \text { CHS prepotentials and field strengths } & 14\end{array}$

$\begin{array}{lll}3.2 & \text { CHS actions } & 16\end{array}$

$\begin{array}{lll}3.3 & \text { Generalised CHS models } & 17\end{array}$

$\begin{array}{ll}3.4 \text { Degauging } & 19\end{array}$

4 Conformal higher-spin models in four dimensions $\quad 19$

4.1 CHS prepotentials and field strengths 20

$\begin{array}{lll}4.2 & \text { CHS actions } & 22\end{array}$

4.3 Generalised CHS models 24

$\begin{array}{lll}4.4 & \text { Degauging } & 27\end{array}$

5 SCHS theories in three dimensions $\quad 29$

$5.1 \mathcal{N}=1$ SCHS theories 31

$5.2 \mathcal{N}=2$ SCHS theories 33

$5.3 \mathcal{N}=3$ SCHS gauge prepotentials $\quad 35$

6 SCHS theories in four dimensions $\quad 35$

$\begin{array}{llr}7 & \text { Concluding comments } & 39\end{array}$

$\begin{array}{ll}\text { A 3D notation and conventions } & 40\end{array}$

B Converting between conventions $\quad 42$

C Properties of the generalised HS Cotton tensor 42

$\begin{array}{ll}\text { D Integration by parts } & 44\end{array}$

$\begin{array}{ll}\text { E Conformal gravitino model } & 46\end{array}$

$\begin{array}{lll}\text { F Conformal graviton model } & 48\end{array}$ 


\section{Introduction}

The concept of conformal higher-spin (CHS) theory was introduced by Fradkin and Tseytlin [1] in 1985 as a generalisation of Maxwell's electrodynamics and conformal gravity in four dimensions. Since then there has been much interest in CHS theories in diverse dimensions, see [2-10] for an incomplete list of works published within a quarter-century after [1]. This interest has truly exploded in the last decade and, unfortunately, it is hardly possible to list all relevant publications (although comments on the literature will be given in the main body). Among the attractive features of CHS theories are the following: (i) maximal spin-s gauge symmetry consistent with locality [1]; (ii) natural connection to the AdS/CFT correspondence [6]; (iii) Lagrangian formulation for a complete interacting bosonic CHS theory [7]; and (iv) interesting quantum properties [11-15].

Off-shell $\mathcal{N}=1$ superconformal higher-spin (SCHS) multiplets in four dimensions were briefly discussed, in the framework of supercurrent multiplets, by Howe, Stelle and Townsend [16] in 1981, a few years before Fradkin and Tseytlin [1] constructed the free CHS actions. It was only in 2017 that the higher-spin gauge prepotentials (describing superspin- $\left(s+\frac{1}{2}\right)$ multiplet, with $\left.s=2,3, \ldots\right)$ introduced in [16] and more general off-shell gauge supermultiplets were finally used to construct free $\mathcal{N}=1$ SCHS actions [17]. Parallel studies in three dimensions $(3 D)$ describing SCHS multiplets and the corresponding ChernSimons actions were conducted in [18-20] and [21,22] for the cases $\mathcal{N}=1$ and $\mathcal{N}=2$, respectively. These $3 D$ and $4 D$ off-shell constructions open the possibility to develop a manifestly supersymmetric setting for SCHS theories first advocated by Fradkin and Linetsky $[2,5]$ in the component approach. It also becomes feasible, as was briefly discussed in [17], to formulate an interacting SCHS theory by developing a superfield analogue of the bosonic CHS theory in even dimensions constructed in full generality by Segal [7] (as an extension of his earlier work [23]), in agreement with Tseytlin's observations [6]. ${ }^{1}$

An important feature of the approach advocated in [17] is that it provides a new avenue to study the problem of consistent propagation of conformal higher-spin fields on curved backgrounds. It is believed that a gauge-invariant action for conformal fields of spin $s>2$ may be defined only if the background metric is a solution of the equation of motion for conformal gravity, which means that the Bach tensor is equal to zero. However, even the simplest $s=3$ case has not yet been studied in full generality [26-29]. When dealing with $\mathcal{N}=1$ SCHS theories in curved backgrounds, the gravitational field belongs to the socalled Weyl multiplet $[30,31]$ which also contains a conformal gravitino and a $\mathrm{U}(1)$ gauge field. It appears that consistent propagation of SCHS multiplets in such a background may be defined if the corresponding super Bach tensor [32, 33] vanishes ${ }^{2}$ and, therefore, the background Weyl multiplet is a solution to the equations of motion for conformal supergravity. So far explicit calculations have been carried out only for the superconformal gravitino multiplet in a supergravity background [17].

\footnotetext{
${ }^{1}$ For more recent derivations of the Segal theory see, e.g., [24, 25] and references therein.

${ }^{2}$ The terminology "super Bach tensor" was introduced in [17]. In linearised conformal supergravity the super Bach tensor was first computed by Ferrara and Zumino [34].
} 
The $3 D$ story is considerably simpler and more complete as far as the issue of consistent propagation of higher-spin fields on curved backgrounds is concerned. The equation of motion for conformal gravity requires the Cotton tensor to vanish [35-37], and therefore curved spacetime is conformally flat. In off-shell $\mathcal{N}$-extended conformal supergravity, with $1 \leq \mathcal{N} \leq 6$, the superfield Euler-Lagrange equation states that the super Cotton tensor is equal to zero [38-40], and therefore curved superspace is conformally flat. It was shown in $[20,22]$ that a gauge-invariant action exists for every conformal higher-spin (super)field on arbitrary conformally flat backgrounds for the cases $\mathcal{N}=0,1,2$. These results may be naturally extended (at least) to the $\mathcal{N}=3$ case.

This paper is a continuation of the research program initiated in [17, 20, 22]. Our main goal will be to develop a formalism with manifest local (super)conformal symmetry. This will allow us, in particular, to elaborate on several constructions that were only sketched in $[17,20,22]$.

Two years ago, ref. [17] proposed off-shell $4 D \mathcal{N}=1$ superconformal higher-spin models in arbitrary conformally flat supergravity backgrounds. Technical details of the corresponding formulation were not spelled out in [17] since the linearised higher-spin (super) Weyl tensors were explicitly given in terms of the gauge prepotentials only for the models describing conformal superspin values $s=1, \frac{3}{2}, \frac{5}{2}$.

More recently, off-shell actions were derived for linearised $3 D \mathcal{N}=0,1$ (super)conformal higher-spin gravity in general conformally flat (super)gravity backgrounds [20]. This construction was also extended to the $\mathcal{N}=2$ superconformal case in [22]. As in the $4 D$ analysis of [17], technical details of the $3 D$ formulations were not given, since closed-form expressions for the linearised higher-spin (super) Cotton tensors in terms of the gauge prepotentials were not known.

In this work we fill the technical gaps in the constructions of $[17,20,22]$. In particular, we explicitly construct CHS models that are Weyl invariant in any curved $4 D$ spacetime or any conformally flat $3 D$ spacetime. In both dimensions the higher-spin gauge invariance of these models holds only in conformally flat spacetimes. Supersymmetric extensions of the models are also given. In addition, by extending the depth of the higher-spin gauge symmetry, we construct novel generalisations of the proposed CHS models whose Weyl and gauge invariance hold under the same conditions.

Of central importance to our approach are (i) the formulation of conformal gravity as the gauge theory of the conformal group [30]; and (ii) the off-shell formulations for conformal supergravity in diverse dimensions known as conformal superspace [38, 39, 41-44], an approach pioneered by Butter in the $4 D$ case [41, 42]. Since superfield techniques are not well known within the higher-spin community, and also since the conformal superspace approach is still familiar only to a limited number of superspace practitioners, the details of our approach and its application to CHS theory will be presented from a nonsupersymmetric point of view. Therefore, the majority of this paper will be devoted to non-supersymmetric CHS models and their supersymmetric counterparts will be presented at the end with the technical details being simply sketched.

This paper is organised as follows. In section 2 we review the formulation of conformal gravity in $D>2$ dimensions as the gauge theory of the conformal group. Section 3 is 
devoted to $3 D$ CHS theories in curved backgrounds. Section 4 discusses $4 D$ CHS theories in curved backgrounds. Supersymmetric extensions are studied in sections 5 and 6 . Concluding comments are given in section 7 . The main body of the paper is accompanied by six technical appendices. Appendix A and B present some of the various conventions that we adopt. Proofs for several properties of the higher-spin generalised Cotton tensors are provided in appendix C. Appendix D examines the issue of integration by parts in the conformal space. Appendices $\mathrm{E}$ and $\mathrm{F}$ discuss the construction of conformal spin $s=3 / 2$ and $s=2$ models that are gauge invariant in any $4 D$ Bach-flat spacetime.

Before turning to the main body of this paper, several comments are in order regarding the existence of different ways to describe conformal higher-spin fields. They differ only in the sector of purely gauge degrees of freedom (compensators) that can be eliminated algebraically by applying local symmetry transformations without derivatives. The original Fradkin-Tseytlin model [1] for a conformal field of integer spin $s>1$ is described in terms of a symmetric rank-s tensor field $\boldsymbol{h}_{a_{1} \ldots a_{s}}=\boldsymbol{h}_{\left(a_{1} \ldots a_{s}\right)} \equiv \boldsymbol{h}_{a(s)}$ with the gauge transformation law

$$
\delta \boldsymbol{h}_{a_{1} \ldots a_{s}}=\partial_{\left(a_{1}\right.} \xi_{\left.a_{2} \ldots a_{s}\right)}+\eta_{\left(a_{1} a_{2}\right.} \lambda_{\left.a_{3} \ldots a_{s}\right)}, \quad \eta^{b c} \xi_{b c a_{1} \ldots a_{s-3}}=0,
$$

where both gauge parameters $\xi_{a(s-1)}$ and $\lambda_{a(s-2)}$ are symmetric, and $\xi_{a(s-1)}$ is in addition traceless. ${ }^{3}$ It is natural to interpret the gauge symmetries generated by $\xi_{a(s-1)}$ and $\lambda_{a(s-2)}$ for $s>2$ as linearised higher-spin gauge and "generalised Weyl" transformations, respectively. The $\lambda$-gauge freedom in (1.1) may be used to make the gauge field $\boldsymbol{h}_{a(s)}$ traceless by requiring

$$
\boldsymbol{h}_{a(s)}=h_{a(s)}, \quad \eta^{b c} h_{b c a(s-2)}=0 .
$$

If one switches to the two-component spinor notation and introduces

$$
h_{a(s)} \rightarrow h_{\alpha_{1} \ldots \alpha_{s} \dot{\alpha}_{1} \ldots \dot{\alpha}_{s}}:=\left(\sigma^{a_{1}}\right)_{\alpha_{1} \dot{\alpha}_{1}} \ldots\left(\sigma^{a_{s}}\right)_{\alpha_{s} \dot{\alpha}_{s}} h_{a_{1} \ldots a_{s}},
$$

then the field $h_{\alpha_{1} \ldots \alpha_{s} \dot{\alpha}_{1} \ldots \dot{\alpha}_{s}}$ proves to be symmetric in its undotted indices and, separately, in its dotted indices, $h_{\alpha_{1} \ldots \alpha_{s} \dot{\alpha}_{1} \ldots \dot{\alpha}_{s}}=h_{\left(\alpha_{1} \ldots \alpha_{s}\right)\left(\dot{\alpha}_{1} \ldots \dot{\alpha}_{s}\right)} \equiv h_{\alpha(s) \dot{\alpha}(s)}$. In accordance with (1.1), the gauge transformation of $h_{\alpha(s) \dot{\alpha}(s)}$ is

$$
\delta h_{\alpha_{1} \ldots \alpha_{s} \dot{\alpha}_{1} \ldots \dot{\alpha}_{s}}=\partial_{\left(\alpha _ { 1 } \left(\dot{\alpha}_{1}\right.\right.} \xi_{\left.\left.\alpha_{2} \ldots \alpha_{s}\right) \dot{\alpha}_{2} \ldots \dot{\alpha}_{s}\right)} .
$$

It is natural to think of $h_{a(s)}$ (or equivalently $h_{\alpha(s) \dot{\alpha}(s)}$ ) as the genuine conformal spin- $s$ gauge field, due to several reasons. Firstly, one can consistently define $h_{\alpha(s) \dot{\alpha}(s)}$ to be a conformal primary field, see section 4. Secondly, the other degrees of freedom contained in $\boldsymbol{h}_{a(s)}$ are purely gauge ones, and as such they may become essential only at the nonlinear level. Finally, the nonlinear conformal higher-spin theory of [7] is formulated in terms of the fields $h_{a(s)}$, with $s=0,1,2, \ldots$, in the $4 D$ case.

In principle, one may instead use Fronsdal's doubly traceless spin- $s$ gauge field $[45,46]$

$$
\mathfrak{h}_{a_{1} \ldots a_{s}}=h_{a_{1} \ldots a_{s}}+\eta_{\left(a_{1} a_{2}\right.} \varphi_{\left.a_{3} \ldots a_{s}\right)}, \quad \eta^{b c} \varphi_{b c a(s-4)}=0,
$$

\footnotetext{
${ }^{3}$ The gauge transformation law (1.1) is often generalised by removing the condition $\eta^{b c} \xi_{\text {bca(s-3) }}=0$ imposed on the parameter $\xi_{a(s-1)}$. However the resulting transformation law is equivalent to (1.1) with a modified algebraic parameter $\lambda_{a(s-2)}$.
} 
to describe conformal spin- $s$ dynamics. In such an approach $\varphi_{a(s-2)}$ is a compensator. The gauge transformation law of $\mathfrak{h}_{a(s)}$ is given by

$$
\delta \mathfrak{h}_{a(s)}=\partial_{\left(a_{1}\right.} \xi_{\left.a_{2} \ldots a_{s}\right)}+\eta_{\left(a_{1} a_{2}\right.} \tilde{\lambda}_{\left.a_{3} \ldots a_{s}\right)}, \quad \eta^{b c} \xi_{b c a(s-3)}=0, \quad \eta^{b c} \tilde{\lambda}_{b c a(s-4)}=0 .
$$

It is clear that the compensator $\varphi_{a(s-2)}$ may be gauged away by applying a $\tilde{\lambda}$-transformation, and then we are back to the formulation in terms of $h_{a(s)}$.

Another description of conformal spin- $s$ dynamics is obtained by employing Vasiliev's frame field [47, 48]

$$
\boldsymbol{e}_{m, a_{1} \ldots a_{s-1}}=\boldsymbol{e}_{m,\left(a_{1} \ldots a_{s-1}\right)}, \quad \eta^{b c} \boldsymbol{e}_{m, b c a(s-3)}=0 .
$$

In addition to a higher-spin $\xi$-transformation, $\delta \boldsymbol{e}_{m, a(s-1)}=\partial_{m} \xi_{a(s-1)}$, there are two additional local symmetries in this setting. These are generalised Lorentz and Weyl transformations, which do not involve derivatives and allow one to gauge away two compensating degrees of freedom contained in $\boldsymbol{e}_{m, a(s-1)}$ by imposing the gauge condition that $\boldsymbol{e}_{m, a(s-1)}$ is completely symmetric, $\boldsymbol{e}_{m, a(s-1)}=h_{m a(s-1)}$.

Not all of the field realisations discussed above originate in the $4 D \mathcal{N}=1$ superconformal setting. We recall that the conformal superspin- $\left(s+\frac{1}{2}\right)$ prepotential $[16,17]$ $H_{\alpha(s) \dot{\alpha}(s)}:=H_{\alpha_{1} \ldots \alpha_{s} \dot{\alpha}_{1} \ldots \dot{\alpha}_{s}}(\theta, \bar{\theta})$ is a real superfield, which is symmetric in its undotted indices and, independently, in its dotted indices. The gauge transformation law of $H_{\alpha(s) \dot{\alpha}(s)}$ is

$$
\delta H_{\alpha_{1} \ldots \alpha_{s} \dot{\alpha}_{1} \ldots \dot{\alpha}_{s}}=\bar{D}_{\left(\dot{\alpha}_{1}\right.} \Lambda_{\left.\alpha_{1} \ldots \alpha_{s} \dot{\alpha}_{2} \ldots \dot{\alpha}_{s}\right)}-D_{\left(\alpha_{1}\right.} \bar{\Lambda}_{\left.\alpha_{2} \ldots \alpha_{s}\right) \dot{\alpha}_{1} \ldots \dot{\alpha}_{s}}
$$

with the gauge parameter $\Lambda_{\alpha(s) \dot{\alpha}(s-1)}$ being unconstrained. For the $s=1$ case this transformation law corresponds to linearised conformal supergravity [34]. The gauge freedom makes it possible to choose a Wess-Zumino gauge

$$
\begin{aligned}
H_{\alpha_{1} \ldots \alpha_{s} \dot{\alpha}_{1} \ldots \dot{\alpha}_{s}}(\theta, \bar{\theta})= & \theta^{\beta} \bar{\theta}^{\dot{\beta}} \boldsymbol{e}_{\beta, \alpha_{1} \ldots \alpha_{s} \dot{\beta}, \dot{\alpha}_{1} \ldots \dot{\alpha}_{s}}+\bar{\theta}^{2} \theta^{\beta} \boldsymbol{\psi}_{\beta, \alpha_{1} \ldots \alpha_{s} \dot{\alpha}_{1} \ldots \dot{\alpha}_{s}}-\theta^{2} \bar{\theta}^{\dot{\beta}} \overline{\boldsymbol{\psi}}_{\alpha_{1} \ldots \alpha_{s} \dot{\beta}, \dot{\alpha}_{1} \ldots \dot{\alpha}_{s}} \\
& +\theta^{2} \bar{\theta}^{2} h_{\alpha_{1} \ldots \alpha_{s} \dot{\alpha}_{1} \ldots \dot{\alpha}_{s}},
\end{aligned}
$$

where the bosonic fields $\boldsymbol{e}_{\beta, \alpha(s) \dot{\beta}, \dot{\alpha}(s)}=\left(\sigma^{m}\right)_{\beta \dot{\beta}} \boldsymbol{e}_{m, \alpha(s) \dot{\alpha}(s)}$ and $h_{\alpha(s) \dot{\alpha}(s)}$ are real. In the Wess-Zumino gauge (1.9), we stay with a restricted set of local transformations (1.8). It is not difficult to check that the transformation law of $\boldsymbol{e}_{m, \alpha(s) \dot{\alpha}(s)}$ coincides with that of the spin- $(s+1)$ frame field [47, 48]. The gauge transformation of $h_{\alpha(s) \dot{\alpha}(s)}$ coincides with (1.4). The fermionic field $\boldsymbol{\psi}_{\beta, \alpha(s) \dot{\alpha}(s)}$ and its conjugate in (1.9) describe the conformal spin- $\left(s+\frac{1}{2}\right)$ gauge field. This field realisation coincides neither with the Fradkin-Tseytlin conformal spin- $\left(s+\frac{1}{2}\right)$ field [1] nor with Vasiliev's fermionic frame field [47, 48].

The residual gauge freedom (1.8), which preserves the Wess-Zumino gauge (1.9), contains algebraic local transformations that can be used to eliminate the compensators such that $H_{\alpha(s) \dot{\alpha}(s)}$ takes the form [17]

$$
\begin{aligned}
H_{\alpha_{1} \ldots \alpha_{s} \dot{\alpha}_{1} \ldots \dot{\alpha}_{s}}(\theta, \bar{\theta})= & \theta^{\beta} \bar{\theta}^{\dot{\beta}} h_{\left(\beta \alpha_{1} \ldots \alpha_{s}\right)\left(\dot{\beta} \dot{\alpha}_{1} \ldots \dot{\alpha}_{s}\right)}+\bar{\theta}^{2} \theta^{\beta} \psi_{\left(\beta \alpha_{1} \ldots \alpha_{s}\right) \dot{\alpha}_{1} \ldots \dot{\alpha}_{s}} \\
& -\theta^{2} \bar{\theta}^{\dot{\beta}} \bar{\psi}_{\alpha_{1} \ldots \alpha_{s}\left(\dot{\beta} \dot{\alpha}_{1} \ldots \dot{\alpha}_{s}\right)}+\theta^{2} \bar{\theta}^{2} h_{\alpha_{1} \ldots \alpha_{s} \dot{\alpha}_{1} \ldots \dot{\alpha}_{s}}
\end{aligned}
$$


The gauge transformation of $\psi_{\alpha(s+1) \dot{\alpha}(s)}$ is

$$
\delta \psi_{\alpha_{1} \ldots \alpha_{s+1} \dot{\alpha}_{1} \ldots \dot{\alpha}_{s}}=\partial_{\left(\alpha _ { 1 } \left(\dot{\alpha}_{1}\right.\right.} \rho_{\left.\left.\alpha_{2} \ldots \alpha_{s+1}\right) \dot{\alpha}_{2} \ldots \dot{\alpha}_{s}\right)} .
$$

It is natural to think of field $\psi_{\alpha(s+1) \dot{\alpha}(s)}$ and its conjugate as the genuine conformal spin$\left(s+\frac{1}{2}\right)$ gauge field.

\section{Conformal geometry}

Conformal (super)gravity as the gauge theory of the (super)conformal group was constructed long ago [30, 31, 37, 49], see [1, 50] for pedagogical reviews. In this section we give a brief review of the formulation for conformal gravity in $D>2$ dimensions following [38]. This setting is known to be ideal for extensions to the superspace formulations for conformal supergravity in diverse dimensions [39, 41-43]. It also turns out to be useful in the framework of higher-spin (super)conformal dynamics, as will be shown below.

\subsection{Gauging the conformal algebra}

The conformal algebra in $D>2$ dimensions, $\mathfrak{s o}(D, 2)$, consists of the translation $\left(P_{a}\right)$, Lorentz $\left(M_{a b}\right)$, special conformal $\left(K_{a}\right)$ and dilatation $(\mathbb{D})$ generators. The non-vanishing commutators are given by

$$
\begin{aligned}
{\left[M_{a b}, M_{c d}\right] } & =2 \eta_{c[a} M_{b] d}-2 \eta_{d[a} M_{b] c}, & & \\
{\left[M_{a b}, P_{c}\right] } & =2 \eta_{c[a} P_{b]}, & & {\left[\mathbb{D}, P_{a}\right]=P_{a}, } \\
{\left[M_{a b}, K_{c}\right] } & =2 \eta_{c[a} K_{b]}, & & {\left[\mathbb{D}, K_{a}\right]=-K_{a}, } \\
{\left[K_{a}, P_{b}\right] } & =2 \eta_{a b} \mathbb{D}+2 M_{a b} . & &
\end{aligned}
$$

The generators $M_{a b}, K_{a}$ and $\mathbb{D}$ span a subalgebra of $\mathfrak{s o}(D, 2)$ and are collectively referred to as $X_{\underline{a}}$. In contrast, we denote the generators of the full algebra by $X_{\tilde{a}}$. Then, the commutation relations (2.1) may be rewritten as follows ${ }^{4}$

$$
\begin{aligned}
{\left[X_{\underline{a}}, X_{\underline{b}}\right] } & =-f_{\underline{a} \underline{b}} \underline{\underline{c}} X_{\underline{c}}, \\
{\left[X_{\underline{a}}, P_{b}\right] } & =-f_{\underline{a} b} b^{\underline{c}} X_{\underline{c}}-f_{\underline{a} b}{ }^{c} P_{c}
\end{aligned}
$$

where $f_{\tilde{a} \tilde{b}}^{\tilde{c}}$ are the structure constants whose non-vanishing components are:

$$
\begin{aligned}
f_{M_{a b}, M_{c d}}{ }^{M_{f g}} & =4 \eta_{a[c} \delta_{d]}^{[f} \delta_{b}^{g]}-4 \eta_{b[c} \delta_{d]}^{[f} \delta_{a}^{g]}, & & \\
f_{M_{a b}, P_{c}}{ }^{P_{d}} & =-2 \eta_{c[a} \delta_{b]}^{d}, & f_{\mathbb{D}, P_{a}}{ }^{P_{b}} & =-\delta_{a}^{b}, \\
f_{M_{a b}, K_{c}}{ }^{K_{d}} & =-2 \eta_{c[a} \delta_{b]}^{d}, & f_{\mathbb{D}, K_{a}}{ }^{K_{b}} & =\delta_{a}^{b}, \\
f_{K_{a}, P_{b}}{ }^{\mathbb{D}} & =-2 \eta_{a b}, & f_{K_{a}, P_{b}}{ }^{M_{c d}} & =-4 \delta_{a}^{[c} \delta_{b}^{d]} .
\end{aligned}
$$

\footnotetext{
${ }^{4}$ We adopt the convention whereby a factor of $1 / 2$ is inserted when summing over pairs of antisymmetric indices. For example, $f_{\underline{a b}}^{\underline{c}} X_{\underline{c}}=f_{\underline{a b}} K_{c} K_{c}+\frac{1}{2} f_{\underline{a b}}{ }^{M_{c d}} M_{c d}+\ldots$
} 
The structure constants satisfy the Jacobi identities

$$
f_{[\tilde{a} \tilde{b}}^{\tilde{b}} f_{\tilde{c}] \tilde{d}}^{\tilde{e}}=0 .
$$

Let $\mathcal{M}^{D}$ be a $D$-dimensional spacetime parameterised by local coordinates $x^{m}$, where $m=0,1, \ldots, D-1$. To gauge the conformal algebra, we associate with each generator $X_{\underline{a}}$ a connection one-form, $\omega^{\underline{a}}=\mathrm{d} x^{m} \omega_{m} \underline{a}$, and with $P_{a}$ the vielbein $e^{a}=\mathrm{d} x^{m} e_{m}{ }^{a}$. We denote by $\mathcal{H}$ the gauge group generated by $X_{\underline{a}}$ and postulate that $e^{a}$ and $\omega^{\underline{a}}$ transform under $\mathcal{H}$ as

$$
\begin{aligned}
\delta_{\mathcal{H}} e^{a} & =e^{b} \Lambda^{\underline{c}} f_{\underline{c} b}{ }^{a}, \\
\delta_{\mathcal{H}} \omega^{\underline{a}} & =\mathrm{d} \Lambda^{\underline{a}}+e^{b} \Lambda^{\underline{c}} f_{\underline{\underline{c}} b^{\underline{a}}}+\omega^{\underline{b}} \Lambda^{\underline{c}} f_{\underline{\underline{c} b}} \underline{\underline{a}},
\end{aligned}
$$

with gauge parameter $\Lambda^{\underline{a}}$.

Given a field $\Phi$ (with its indices suppressed), we say that $\Phi$ is $\mathcal{H}$-covariant if it transforms under the action of $\mathcal{H}$ with no derivative on the parameter, $\delta_{\mathcal{H}} \Phi=\Lambda_{\underline{a}}^{\underline{a}} X_{\underline{a}} \Phi$. In addition, if $\Phi$ satisfies

$$
K_{a} \Phi=0, \quad \mathbb{D} \Phi=\Delta \Phi,
$$

it is called a primary field of dimension (or Weyl weight) $\Delta$.

It is clear that $\partial_{m} \Phi$ is no longer $\mathcal{H}$-covariant. We are therefore led to introduce a covariant derivative according to

$$
\nabla_{m}=\partial_{m}-\omega_{m} \underline{a} X_{\underline{a}} .
$$

It follows from (2.5) that $\nabla_{a} \Phi=e_{a}{ }^{m} \nabla_{m} \Phi$ transforms covariantly,

$$
\delta_{\mathcal{H}}\left(\nabla_{a} \Phi\right)=\Lambda^{\underline{b}} \nabla_{a} X_{\underline{b}} \Phi-\Lambda^{\underline{b}} f_{\underline{b} a}{ }^{c} \nabla_{c} \Phi-\Lambda^{\underline{b}} f_{\underline{b} a} \underline{\underline{c}} X_{\underline{c}} \Phi .
$$

From eq. (2.8) we can deduce the commutation relations of $X_{\underline{a}}$ with $\nabla_{a}$,

$$
\left[X_{\underline{a}}, \nabla_{b}\right]=-f_{\underline{a} b}{ }^{\underline{c}} X_{\underline{c}}-f_{\underline{a} b}^{c} \nabla_{c} .
$$

Comparing this with $(2.2 \mathrm{~b})$ we see that $X_{\underline{a}}$ satisfies the same commutation relations with $\nabla_{b}$ as it does with $P_{b}$. However, unlike the translation generators $P_{a}$, the commutator of two covariant derivatives is not zero but is given by

$$
\left[\nabla_{a}, \nabla_{b}\right]=-\mathcal{T}_{a b}{ }^{c} \nabla_{c}-\mathcal{R}_{a b} \underline{c}^{\underline{c}} .
$$

In eq. (2.10), $\mathcal{T}_{a b}{ }^{c}$ and $\mathcal{R}_{a b^{\underline{c}}}$ are the torsion and curvature tensors respectively,

$$
\begin{aligned}
& \mathcal{T}_{a b}^{c}=-\mathscr{C}_{a b}{ }^{c}+2 \omega_{\left[a^{a}\right.} f_{b] \underline{d}}^{c}, \\
& \left.\mathcal{R}_{a b^{\underline{c}}}=-\mathscr{C}_{a b}{ }^{c} \omega_{c^{\underline{c}}}+2 \omega_{\left[a^{\underline{d}}\right.} f_{b] \underline{d}^{\underline{c}}}+\omega_{\left[a^{\underline{e}}\right.} \omega_{b]} \underline{\underline{d}} f_{\underline{d e}} \underline{c}^{\underline{c}}+2 e_{[a} \omega_{b]}\right]^{\underline{c}},
\end{aligned}
$$

where $e_{a}=e_{a}{ }^{m} \partial_{m}$ is the inverse vielbein and the anholonomy coefficients, $\mathscr{C}_{a b}{ }^{c}$, are given by

$$
\mathscr{C}_{a b}{ }^{c}=\left(e_{a} e_{b}{ }^{m}-e_{b} e_{a}{ }^{m}\right) e_{m}{ }^{c} .
$$

The definitions (2.7) and (2.10) differ from those used in some previous publications. See appendix $\mathrm{B}$ for a dictionary to convert between these conventions. 
Using the transformation rules (2.5) and the Jacobi identities (2.4), we find that the torsion and curvature tensors (2.11) transform covariantly under $\mathcal{H}$ according to

$$
\begin{aligned}
\delta_{\mathcal{H}} \mathcal{T}_{a b}{ }^{c} & =\mathcal{T}_{a b}{ }^{d} \Lambda^{\underline{e}} f_{\underline{e} d}{ }^{c}-2 \Lambda^{\underline{a}} f_{\underline{a}[a}{ }^{d} \mathcal{T}_{b] d}{ }^{c}, \\
\delta_{\mathcal{H}} \mathcal{R}_{a b} \underline{\underline{c}} & =\mathcal{R}_{a b} \Lambda^{\underline{e}} f_{\underline{d e}}^{\underline{\underline{c}}}+2 \Lambda^{\underline{d}} f_{\underline{d}[a}{ }^{e} \mathcal{R}_{b] e^{\underline{c}}}+\mathcal{T}_{a b}{ }^{e} \Lambda^{\underline{f}} f_{\underline{f}} e^{\underline{c}} .
\end{aligned}
$$

In this formulation infinitesimal general coordinate transformations, generated by a local parameter $\xi^{a}$, are not covariant with respect to $\mathcal{H}$. To remedy this, they must be supplemented by an additional $\mathcal{H}$-transformation with gauge parameter $\Lambda^{\underline{a}}=\xi^{a} \omega_{a}^{\underline{a}}$,

$$
\delta_{\mathrm{cgct}}\left(\xi^{a}\right)=\delta_{\mathrm{gct}}\left(\xi^{a}\right)-\delta_{\mathcal{H}}\left(\xi^{a} \omega_{a^{\underline{a}}}\right) .
$$

It follows that such transformations act on fields $\Phi$ (with all indices Lorentz) as $\delta_{\text {cgct }} \Phi=$ $\xi^{a} \nabla_{a} \Phi$. The conformal gravity gauge group, denoted by $\mathcal{G}$, is then generated by the set of operators $\left(\nabla_{a}, X_{\underline{a}}\right)$ under which $\Phi$ transforms as

$$
\delta_{\mathcal{G}} \Phi=\mathcal{K} \Phi, \quad \mathcal{K}=\xi^{b} \nabla_{b}+\Lambda^{\underline{b}} X_{\underline{b}} .
$$

Finally, the gauge transformation of $\nabla_{a}$ under $\mathcal{G}$ proves to obey the relation

$$
\delta_{\mathcal{G}} \nabla_{a}=\left[\mathcal{K}, \nabla_{a}\right]
$$

provided we interpret

$$
\nabla_{a} \xi^{b}:=e_{a} \xi^{b}+\omega_{a} \underline{\underline{c}} \xi^{d} f_{d \underline{c}}^{b}, \quad \nabla_{a} \Lambda^{\underline{b}}:=e_{a} \Lambda^{\underline{b}}+\omega_{a} \underline{\underline{c}} \xi^{d} f_{d \underline{\underline{c}}}^{\underline{b}}+\omega_{a} \underline{\underline{c}} \Lambda^{\underline{d}} f_{\underline{d c}}^{\underline{b}} .
$$

Through this procedure the entire conformal algebra has been gauged in such a way that the generators $X_{\underline{a}}$ act on $\nabla_{a}$ in the same way as they do on $P_{a}$.

\subsection{Conformal gravity}

The covariant derivatives given by eq. (2.7) are

$$
\nabla_{a}=e_{a}-\frac{1}{2} \hat{\omega}_{a}^{b c} M_{b c}-\mathfrak{f}_{a}^{b} K_{b}-\mathfrak{b}_{a} \mathbb{D}
$$

where $\hat{\omega}_{a}^{b c}, \mathfrak{f}_{a}{ }^{b}$ and $\mathfrak{b}_{a}$ are the Lorentz, special conformal and dilatation connections respectively. They satisfy the commutation relations

$$
\left[\nabla_{a}, \nabla_{b}\right]=-\mathcal{T}_{a b}{ }^{c} \nabla_{c}-\frac{1}{2} \mathcal{R}(M)_{a b}{ }^{c d} M_{c d}-\mathcal{R}(K)_{a b}{ }^{c} K_{c}-\mathcal{R}(\mathbb{D})_{a b} \mathbb{D}
$$

where the torsion and curvatures are

$$
\begin{aligned}
& \mathcal{T}_{a b}{ }^{c}=-\mathscr{C}_{a b}{ }^{c}+2 \hat{\omega}_{[a b]}^{c}+2 \mathfrak{b}_{[a} \delta_{b]}^{c}, \\
& \mathcal{R}(M)_{a b}{ }^{c d}=\hat{R}_{a b}{ }^{c d}+8 \mathfrak{f}_{[a}{ }^{[c} \delta_{b]}{ }^{d]},
\end{aligned}
$$

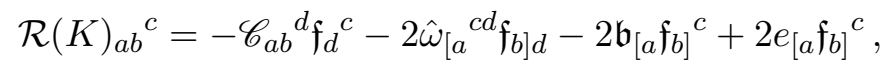

$$
\begin{aligned}
& \mathcal{R}(\mathbb{D})_{a b}=-\mathscr{C}_{a b}{ }^{c} \mathfrak{b}_{c}+4 \mathfrak{f}_{[a b]}+2 e_{[a} \mathfrak{b}_{b]}, \\
& \hat{R}_{a b}{ }^{c d}=-\mathscr{C}_{a b}{ }^{f} \hat{\omega}_{f}^{c d}+2 e_{[a} \hat{\omega}_{b]}^{c d}-2 \hat{\omega}_{[a}^{c f} \hat{\omega}_{b] f}{ }^{d} .
\end{aligned}
$$

Here $\hat{R}_{a b c d}$ is the Riemann tensor corresponding to the spin connection $\hat{\omega}_{a b c}$. 
To ensure that the vielbein is the only independent field in the theory modulo purely gauge degrees of freedom, we have to impose covariant constraints. These constraints are

$$
\begin{aligned}
\mathcal{T}_{a b}{ }^{c} & =0, \\
\eta^{b d} \mathcal{R}(M)_{a b c d} & =0 .
\end{aligned}
$$

Indeed, the conditions (2.21) are preserved by $\mathcal{H}$-transformations, which may be verified through (2.13). The first constraint determines the spin connection in terms of the vielbein and the dilatation connection,

$$
\hat{\omega}_{a b c}=\omega_{a b c}-2 \eta_{a[b} \mathfrak{b}_{c]},
$$

where $\omega_{a b c} \equiv \omega_{a b c}(e)=\frac{1}{2}\left(\mathscr{C}_{a b c}-\mathscr{C}_{a c b}-\mathscr{C}_{b c a}\right)$ is the standard torsion-free Lorentz connection. Similarly, the second constraint fixes the special conformal connection to be

$$
\mathfrak{f}_{a b}=-\frac{1}{2(D-2)} \hat{R}_{a b}+\frac{1}{4(D-1)(D-2)} \eta_{a b} \hat{R},
$$

where $\hat{R}_{a b}=\eta^{c d} \hat{R}_{a c b d}$ is the (non-symmetric) Ricci tensor and $\hat{R}=\eta^{a b} \hat{R}_{a b}$ is the scalar curvature.

Rather than imposing an extra constraint to fix $\mathfrak{b}_{a}$ in terms of the vielbein, we observe that under a $K$-gauge transformation, $\mathfrak{b}_{a}$ transforms as

$$
\delta_{K} \mathfrak{b}_{a}=-2 \Lambda(K)_{a} .
$$

It follows that we may impose the gauge condition

$$
\mathfrak{b}_{a}=0
$$

After this choice, only the vielbein remains as an independent field. The gauge (2.25) breaks the special conformal symmetry. For our purposes, it is desirable to keep this symmetry intact throughout calculations and impose (2.25) only at the end when we wish to extract physically meaningful results. This process is referred to as 'degauging'.

Making use of (2.21) and the Jacobi identity

$$
0=\left[\nabla_{a},\left[\nabla_{b}, \nabla_{c}\right]\right]+\left[\nabla_{b},\left[\nabla_{c}, \nabla_{a}\right]\right]+\left[\nabla_{c},\left[\nabla_{a}, \nabla_{b}\right]\right]
$$

we find that the dilatation field strength vanishes,

$$
\mathcal{R}(\mathbb{D})_{a b}=0,
$$

along with the following Bianchi identities

$$
\begin{aligned}
\mathcal{R}(K)_{[a b c]} & =0, \\
\mathcal{R}(M)_{[a b c] d} & =0, \\
\nabla_{[a} \mathcal{R}(K)_{b c] d} & =0, \\
\nabla_{[a} \mathcal{R}(M)_{b c]} d e-4 \mathcal{R}(K)_{[a b}\left[\delta_{c]} e\right] & =0 .
\end{aligned}
$$


Making use of (2.20e) and (2.22) allows us to decompose $\hat{R}_{a b c d}$ into those terms which depend solely on the vielbein and those involving the dilatation connection,

$$
\begin{aligned}
\hat{R}_{a b c d}= & R_{a b c d}-4 e_{[a} \eta_{b][c} \mathfrak{b}_{d]}-4 \eta_{[c[a} \omega_{b] d]}{ }^{g} \mathfrak{b}_{g}+4 \mathfrak{b}_{[c} \eta_{d][a} \mathfrak{b}_{b]}+2 \eta_{c[a} \eta_{b] d} \mathfrak{b}^{f} \mathfrak{b}_{f} \\
\hat{R}_{a b}= & R_{a b}+(D-2)\left\{e_{a} \mathfrak{b}_{b}-\omega_{a b}{ }^{c} \mathfrak{b}_{c}-\mathfrak{b}_{a} \mathfrak{b}_{b}\right\} \\
& +\eta_{a b}\left\{e_{c} \mathfrak{b}^{c}-\omega^{c}{ }_{c d} \mathfrak{b}^{d}+(D-2) \mathfrak{b}_{c} \mathfrak{b}^{c}\right\} \\
\hat{R}= & R+2(D-1)\left\{e_{a} \mathfrak{b}^{a}-\omega^{a}{ }_{a b} \mathfrak{b}^{b}+\frac{1}{2}(D-2) \mathfrak{b}^{a} \mathfrak{b}_{a}\right\}
\end{aligned}
$$

Here $R_{a b c d}$ is the Riemann tensor associated with the spin connection $\omega_{a b c}$,

$$
R_{a b}^{c d}=2 e_{[a} \omega_{b]}^{c d}-2 \omega_{[a b]}^{f} \omega_{f}^{c d}-2 \omega_{[a}^{c f} \omega_{b] f}^{d},
$$

and $R_{a b}$ and $R$ stand for the corresponding (symmetric) Ricci tensor and scalar curvature, respectively. Inserting the relations (2.29) into the solution to the conformal gravity constraint (2.23) yields

$$
\mathfrak{f}_{a b}=-\frac{1}{2} P_{a b}+\frac{1}{2} \mathfrak{b}_{a} \mathfrak{b}_{b}-\frac{1}{4} \eta_{a b} \mathfrak{b}^{c} \mathfrak{b}_{c}+\frac{1}{2} \omega_{a b}{ }^{c} \mathfrak{b}_{c}-\frac{1}{2} e_{a} \mathfrak{b}_{b}
$$

where $P_{a b}$ is the Schouten tensor,

$$
P_{a b}=\frac{1}{(D-2)}\left(R_{a b}-\frac{1}{2(D-1)} \eta_{a b} R\right) .
$$

Using eqs. (2.29a) and (2.31) allows us to show that the dependence on the dilatation connection drops out of $(2.20 \mathrm{~b})$ and we obtain

$$
\mathcal{R}(M)_{a b c d}=C_{a b c d} .
$$

Here $C_{a b c d}$ is the Weyl tensor,

$$
C_{a b c d}=R_{a b c d}-\frac{2}{(D-2)}\left(R_{a[c} \eta_{d] b}-R_{b[c} \eta_{d] a}\right)+\frac{2}{(D-1)(D-2)} \eta_{a[c} \eta_{d] a} R,
$$

which is a primary field of dimension +2 ,

$$
K_{e} C_{a b c d}=0, \quad \mathbb{D} C_{a b c d}=2 C_{a b c d} .
$$

For further analysis of the constraints, it is necessary to consider separately the choices $D=3$ and $D>3$. In both cases we make use of the Lorentz covariant derivative defined by

$$
\hat{\mathcal{D}}_{a}=e_{a}-\frac{1}{2} \hat{\omega}_{a}^{b c} M_{b c}=\mathcal{D}_{a}+\mathfrak{b}^{c} M_{a c}
$$

where $\mathcal{D}_{a}=e_{a}-\frac{1}{2} \omega_{a}^{b c} M_{b c}$ is the torsion-free Lorentz covariant derivative.

We note that whenever the gauge (2.25) is chosen, all hatted objects coincide with their non-hatted counterparts. In particular

$$
\left.\hat{\mathcal{D}}_{a}\right|_{\mathfrak{b}_{a}=0}=\mathcal{D}_{a},\left.\quad \hat{R}_{a b c d}\right|_{\mathfrak{b}_{a}=0}=R_{a b c d}
$$


and in this gauge we may therefore abandon the hat notation without any ambiguity. Furthermore, in this case it is clear that the conformal covariant derivative takes the form

$$
\nabla_{a}=\mathcal{D}_{a}+\frac{1}{2} P_{a}^{b} K_{b}
$$

Therefore, in any spacetime with vanishing Schouten tensor, the degauging process is trivial.

In the $D>3$ case, it follows from (2.28d) and (2.33) that the special conformal curvature is given by

$$
\mathcal{R}(K)_{a b c}=\frac{1}{2(D-3)} \nabla^{d} C_{a b c d} .
$$

As a result, the algebra of conformal covariant derivatives is

$$
\left[\nabla_{a}, \nabla_{b}\right]=-\frac{1}{2} C_{a b c d} M^{c d}-\frac{1}{2(D-3)} \nabla^{d} C_{a b c d} K^{c} .
$$

It is determined by a single primary tensor field, the Weyl tensor.

The expressions (2.20c) and (2.39) are two equivalent representations for the special conformal curvature. Upon imposing the gauge (2.25) these relations lead to the well-known Bianchi identity

$$
\mathcal{D}^{d} C_{a b c d}=-2(D-3) \mathcal{D}_{[a} P_{b] c} .
$$

From (2.40) it follows that if the spacetime under consideration is conformally flat, then the conformal covariant derivatives commute,

$$
C_{a b c d}=0 \quad \Longrightarrow \quad\left[\nabla_{a}, \nabla_{b}\right]=0 .
$$

This observation will be important for our subsequent analysis.

\subsection{Conformal gravity in three dimensions}

The Weyl tensor vanishes identically in three dimensions. As a result, the Lorentz curvature (2.33) also vanishes and the algebra of conformal covariant derivatives takes the form

$$
\left[\nabla_{a}, \nabla_{b}\right]=-\mathcal{R}(K)_{a b}^{c} K_{c} .
$$

Therefore, all information about conformal geometry is encoded in a single primary field, $\mathcal{R}(K)_{a b c}$, which proves to be proportional to the Cotton tensor, as we now show.

The Lorentz covariant derivative (2.36) allows us to represent (2.20c) as

$$
\mathcal{R}(K)_{a b c}=2 \mathcal{D}_{[a} \mathfrak{f}_{b] c}-2 \mathfrak{b}_{[a} \mathfrak{f}_{b] c}+2 \mathfrak{b}_{c} \mathfrak{f}_{[a b]}+2 \eta_{c[a} \mathfrak{f}_{b] d} \mathfrak{b}^{d}
$$

Using (2.31), one may show that dependence on $\mathfrak{b}_{a}$ in eq. (2.44) drops out such that

$$
\mathcal{R}(K)_{a b c}=-\frac{1}{2} W_{a b c}, \quad W_{a b c}=2 \mathcal{D}_{[a} P_{b] c} .
$$


Here $W_{a b c}$ is the Cotton tensor, which is a primary field of dimension +3 ,

$$
K_{d} W_{a b c}=0, \quad \mathbb{D} W_{a b c}=3 W_{a b c} .
$$

It is useful to introduce the dual of the Cotton tensor,

$$
W_{a b}=\frac{1}{2} \varepsilon_{a c d} W_{b}^{c d}, \quad W_{a b c}=-\varepsilon_{a b d} W_{c}^{d},
$$

which is symmetric and traceless,

$$
W_{a b}=W_{b a}, \quad W_{b}^{b}=0 .
$$

On account of the Bianchi identity $\mathcal{D}^{a} R_{a b}=\frac{1}{2} \mathcal{D}_{b} R$, it is also conserved,

$$
\mathcal{D}^{a} W_{a b}=0 .
$$

The Cotton tensor contains all information about the conformal geometry of $D=$ 3 spacetime, and it vanishes if and only if spacetime is conformally flat. As follows from (2.43), the commutator of conformal covariant derivatives vanishes in the conformally flat case,

$$
W_{a b c}=0 \quad \Longrightarrow \quad\left[\nabla_{a}, \nabla_{b}\right]=0 .
$$

In three dimensions, the Einstein-Hilbert action is known to propagate no local degrees of freedom. However, non-trivial dynamics emerge in topologically massive gravity [35, 36] which is obtained by combining the Einstein-Hilbert action with a Lorentz Chern-Simons term. The latter proves to coincide with the action for $D=3$ conformal gravity $^{5}$ [37]

$$
S_{\mathrm{CG}}=\frac{1}{6} \int \Sigma_{\mathrm{CS}},
$$

where the three-form

$$
\Sigma_{\mathrm{CS}}=\mathcal{R}^{\tilde{b}} \wedge \omega^{\tilde{a}} \Gamma_{\tilde{a} \tilde{b}}+\frac{1}{6} \omega^{\tilde{c}} \wedge \omega^{\tilde{b}} \wedge \omega^{\tilde{a}} f_{\tilde{a} \tilde{b} \tilde{c}}
$$

varies under an infinitesimal $\mathcal{H}$-transformation by an exact form,

$$
\delta_{\mathcal{H}} \Sigma_{\mathrm{CS}}=\mathrm{d}\left(\mathrm{d} \omega^{\tilde{b}} \Lambda^{\tilde{a}} \Gamma_{\tilde{a} \tilde{b}}\right), \quad \Lambda^{\tilde{a}}=\left(0, \Lambda^{\underline{a}}\right) .
$$

Here and in (2.52), $\Gamma_{\tilde{a} \tilde{b}}=f_{\tilde{a} \tilde{d}} \tilde{f}_{\tilde{b} \tilde{c}}^{\tilde{d}}$ is the symmetric non-degenerate Cartan-Killing metric on $\mathfrak{s o}(3,2)$ and $f_{\tilde{a} \tilde{b} \tilde{c}}=f_{\tilde{a} \tilde{b}} \tilde{d} \Gamma_{\tilde{d} \tilde{c}}$ are the totally antisymmetric structure constants, see appendix A. We have also used a unified notation [39] whereby the connection one-forms are written as $\omega^{\tilde{a}}=\left(e^{a}, \omega^{\underline{a}}\right)$ and the curvature two-forms as $\mathcal{R}^{\tilde{a}}=\frac{1}{2} e^{c} \wedge e^{b} \mathcal{R}_{b c}{ }^{\tilde{a}}=\left(\mathcal{T}^{a}, \mathcal{R}^{\underline{a}}\right)$. It should be remarked that we have adopted the super-form conventions for differential forms, see, e.g., [52] for the details.

\footnotetext{
${ }^{5}$ An alternative approach to conformal gravity in three dimensions was developed in [51].
} 
The action for conformal gravity (2.51) can be rewritten in the form

$$
S_{\mathrm{CG}}=\frac{1}{4} \int \mathrm{d}^{3} x e \varepsilon^{a b c}\left\{\hat{R}_{a b}^{f g} \hat{\omega}_{c f g}-\frac{2}{3} \hat{\omega}_{a d}^{e} \hat{\omega}_{b e^{f}} \hat{\omega}_{c f}{ }^{d}+8 \mathfrak{f}_{a b} \mathfrak{b}_{c}\right\}, \quad e:=\operatorname{det}\left(e_{m}{ }^{a}\right) .
$$

Since (2.51) is inert under $K$-transformations up to a total derivative, the dependence on $\mathfrak{b}_{a}$ once again drops out ${ }^{6}$ and the action simplifies to

$$
S_{\mathrm{CG}}=\frac{1}{4} \int \mathrm{d}^{3} x e \varepsilon^{a b c}\left\{R_{a b}^{f g} \omega_{c f g}-\frac{2}{3} \omega_{a d}{ }^{e} \omega_{b e}{ }^{f} \omega_{c f}{ }^{d}\right\} .
$$

Equivalently, one may arrive at equation (2.55) from (2.54) by making use of the special conformal symmetry to impose the gauge (2.25).

The equation of motion derived from the action (2.55) is

$$
W_{a b}=0 \text {. }
$$

Such a conformally flat background has to be used in order to linearise the conformal gravity action (2.55). Before doing that, let us work out how geometric objects change under an infinitesimal deformation of the vielbein,

$$
\delta e_{a}{ }^{m}=h_{a}{ }^{b} e_{b}{ }^{m}, \quad \delta e_{m}{ }^{a}=-e_{m}{ }^{b} h_{b}{ }^{a},
$$

for some second-rank tensor $h_{a b}$. Since the antisymmetric and trace parts of $h_{a b}$ correspond to Lorentz and Weyl transformations, respectively, and we know the behaviour of the geometric objects under such transformations, it suffices to choose $h_{a b}$ to be symmetric and traceless,

$$
h_{a b}=h_{b a}, \quad h_{a}^{a}=0 .
$$

We represent the corresponding change that the covariant derivative suffers as

$$
\delta \mathcal{D}_{a}=h_{a}{ }^{b} \mathcal{D}_{b}-\frac{1}{2} \Xi_{a}{ }^{b c} M_{b c},
$$

where $\mathcal{D}_{a}$ is the background torsion-free Lorentz covariant derivative and $\Xi_{a b c}$ is a deformation of the spin connection. The latter is determined by imposing the torsion-free condition on $\mathcal{D}_{a}^{\prime}=\mathcal{D}_{a}+\delta \mathcal{D}_{a}$, and the result is

$$
\Xi_{a b c}=-2 \mathcal{D}_{[b} h_{c] a} .
$$

Making use of (2.58) leads to the well-known relations

$$
\begin{aligned}
\delta R_{a b c d} & =-2 h^{f}{ }_{[a} R_{b] f c d}-2 \mathcal{D}_{a} \mathcal{D}_{[c} h_{d] b}+2 \mathcal{D}_{b} \mathcal{D}_{[c} h_{d] a} \\
\delta R_{a b} & =2 h^{f}{ }_{(a} R_{b) f}-\square h_{a b}+2 \mathcal{D}^{f} \mathcal{D}_{(a} h_{b) f} \\
\delta R & =2 h^{a b} R_{a b}+2 \mathcal{D}^{a} \mathcal{D}^{b} h_{a b}
\end{aligned}
$$

\footnotetext{
${ }^{6}$ This may be shown explicitly using the relations (2.22), (2.29a) and (2.31).
} 
where $\square=\mathcal{D}^{a} \mathcal{D}_{a}$. The relations (2.58) and (2.59) allow one to read off the deformation $\delta W_{a b}$ of the Cotton tensor. For our subsequent consideration in section 3 it is suitable to give the expression for $\delta W_{a b}$ in the spinor notation. A summary of our spinor conventions is given in appendix A to which the reader is referred for the technical details.

Associated with the traceless Ricci tensor, $R_{a b}-\frac{1}{3} \eta_{a b} R$, and the Cotton tensor, $W_{a b}$, are symmetric rank-four spinors defined by

$$
\begin{aligned}
R_{\alpha \beta \gamma \delta} & =\left(\gamma^{a}\right)_{\alpha \beta}\left(\gamma^{b}\right)_{\gamma \delta}\left(R_{a b}-\frac{1}{3} \eta_{a b} R\right)=R_{(\alpha \beta \gamma \delta)}, \\
W_{\alpha \beta \gamma \delta} & =\left(\gamma^{a}\right)_{\alpha \beta}\left(\gamma^{b}\right)_{\gamma \delta} W_{a b}=W_{(\alpha \beta \gamma \delta)} .
\end{aligned}
$$

The latter can be represented in the form

$$
W_{\alpha \beta \gamma \delta}=\mathcal{D}_{(\alpha}^{\sigma} R_{\beta \gamma \delta) \sigma},
$$

where $\mathcal{D}_{\alpha \beta}=\left(\gamma^{a}\right)_{\alpha \beta} \mathcal{D}_{a}$. The infinitesimal deformation defined by (2.58) and (2.59) may be shown to lead to

$$
\begin{aligned}
& \delta R_{\alpha(4)}=-\mathcal{D}_{\left(\alpha_{1}\right.}{ }^{\beta_{1}} \mathcal{D}_{\alpha_{2}}{ }^{\beta_{2}} h_{\left.\alpha_{3} \alpha_{4}\right) \beta(2)}+\frac{1}{2} R^{\beta(2)}{ }_{\left(\alpha_{1} \alpha_{2}\right.} h_{\left.\alpha_{3} \alpha_{4}\right) \beta(2)}+\frac{1}{6} R h_{\alpha(4)}, \\
& \delta W_{\alpha(4)}=\frac{1}{2} W^{\beta(2)}{ }_{\left(\alpha_{1} \alpha_{2}\right.} h_{\left.\alpha_{3} \alpha_{4}\right) \beta(2)}-\frac{1}{2} \mathcal{D}_{\left(\alpha_{1}\right.}{ }^{\beta_{1}} \mathcal{D}_{\alpha_{2}}{ }^{\beta_{2}} \mathcal{D}_{\alpha_{3}}{ }^{\beta_{3}} h_{\left.\alpha_{4}\right) \beta(3)}-\frac{1}{2} \square \mathcal{D}_{\left(\alpha_{1}\right.}{ }^{\beta_{1}} h_{\left.\alpha_{2} \alpha_{3} \alpha_{4}\right) \beta_{1}} \\
& +\left(\mathcal{D}_{\left(\alpha_{1}\right.}^{\beta_{1}} R_{\alpha_{2} \alpha_{3}}^{\beta_{2} \beta_{3}}\right) h_{\left.\alpha_{4}\right) \beta(3)}+\frac{1}{12}\left(\mathcal{D}_{\left(\alpha_{1}\right.}^{{ }^{\beta_{1}}} R\right) h_{\left.\alpha_{2} \alpha_{3} \alpha_{4}\right) \beta_{1}}-\frac{1}{12} R \mathcal{D}_{\left(\alpha_{1}\right.}{ }^{\beta_{1}} h_{\left.\alpha_{2} \alpha_{3} \alpha_{4}\right) \beta_{1}} \\
& +2 R^{\beta_{1} \beta_{2}}{ }_{\left(\alpha_{1} \alpha_{2}\right.} \mathcal{D}_{\alpha_{3}}{ }^{\beta_{3}} h_{\left.\alpha_{4}\right) \beta(3)}-\frac{3}{4} R^{\beta_{1}}{ }_{\delta\left(\alpha_{1} \alpha_{2}\right.} \mathcal{D}^{\delta \beta_{2}} h_{\left.\alpha_{3} \alpha_{4}\right) \beta(2)},
\end{aligned}
$$

where $h_{\alpha \beta \gamma \delta}=\left(\gamma^{a}\right)_{\alpha \beta}\left(\gamma^{b}\right)_{\gamma \delta} h_{a b}=h_{(\alpha \beta \gamma \delta)}$.

The conformal gravity action (2.55) may be linearised around a background spacetime that is a solution to the equation of motion (2.56). The result is

$$
S_{\mathrm{CG}, \text { linearised }}=-\frac{1}{4} \int \mathrm{d}^{3} x e h^{\alpha(4)} \mathfrak{C}_{\alpha(4)},
$$

where $\mathfrak{C}_{\alpha(4)}=-\delta W_{\alpha(4)}$ and $\delta W_{\alpha(4)}$ is obtained from (2.62b), by setting $W_{\alpha(4)}=0$. The linearised action proves to be conformal (assuming $h_{\alpha(4)}$ to be a primary field of dimension 0 ) as well as it is invariant under the gauge transformations

$$
\delta_{\xi} h_{\alpha(4)}=\mathcal{D}_{\left(\alpha_{1} \alpha_{2}\right.} \xi_{\left.\alpha_{3} \alpha_{4}\right)}
$$

where the gauge parameter $\xi_{\alpha \beta}$ is a primary field of dimension -1 .

\section{Conformal higher-spin models in three dimensions}

In order to describe higher-spin models, it is useful to convert to spinor notation. Then the commutator of two covariant derivatives takes the form

$$
\left[\nabla_{\alpha \beta}, \nabla_{\gamma \delta}\right]=\frac{1}{4} \varepsilon_{\gamma(\alpha} W_{\beta) \delta}{ }^{\rho(2)} K_{\rho(2)}+\frac{1}{4} \varepsilon_{\delta(\alpha} W_{\beta) \gamma}{ }^{\rho(2)} K_{\rho(2)},
$$


and the commutation relations of the other generators of the conformal group with the covariant derivatives are as follows:

$$
\begin{aligned}
{\left[M_{\alpha \beta}, \nabla_{\gamma \delta}\right] } & =\varepsilon_{\gamma(\alpha} \nabla_{\beta) \delta}+\varepsilon_{\delta(\alpha} \nabla_{\beta) \gamma} \\
{\left[\mathbb{D}, \nabla_{\alpha \beta}\right] } & =\nabla_{\alpha \beta}, \\
{\left[K_{\alpha \beta}, \nabla_{\gamma \delta}\right] } & =4 \varepsilon_{\gamma(\alpha} \varepsilon_{\beta) \delta} \mathbb{D}-2 \varepsilon_{\gamma(\alpha} M_{\beta) \delta}-2 \varepsilon_{\delta(\alpha} M_{\beta) \gamma} .
\end{aligned}
$$

The remaining non-vanishing commutators between the generators are given in appendix A.

\subsection{CHS prepotentials and field strengths}

We introduce conformal higher-spin gauge fields by extending the discussion in [20]. Consider a real totally symmetric rank- $n$ spinor field $h_{\alpha(n)}:=h_{\alpha_{1} \ldots \alpha_{n}}=h_{\left(\alpha_{1} \ldots \alpha_{n}\right)}$ which is primary and of dimension $(2-n / 2)$,

$$
K_{\beta(2)} h_{\alpha(n)}=0, \quad \mathbb{D} h_{\alpha(n)}=\left(2-\frac{n}{2}\right) h_{\alpha(n)} .
$$

Its dimension is uniquely fixed by requiring $h_{\alpha(n)}$ to be defined modulo gauge transformations of the form

$$
\delta_{\xi} h_{\alpha(n)}=\nabla_{\left(\alpha_{1} \alpha_{2}\right.} \xi_{\left.\alpha_{3} \ldots \alpha_{n}\right)}
$$

with the real gauge parameter $\xi_{\alpha(n-2)}$ being also primary. We say that $h_{\alpha(n)}$ is a conformal spin- $\frac{n}{2}$ gauge field.

Starting from $h_{\alpha(n)}$ one may construct a descendant $\mathfrak{C}_{\alpha(n)}(h)$, known as the higher-spin Cotton tensor, with the following properties:

1. $\mathfrak{C}_{\alpha(n)}$ is of the form $\mathcal{A} h_{\alpha(n)}$, where $\mathcal{A}$ is a linear differential operator involving the covariant derivatives, the Cotton tensor $W_{\alpha(4)}$, and its covariant derivatives.

2. $\mathfrak{C}_{\alpha(n)}$ is a primary field of dimension $(1+n / 2)$,

$$
K_{\beta(2)} \mathfrak{C}_{\alpha(n)}=0, \quad \mathbb{D} \mathfrak{C}_{\alpha(n)}=\left(1+\frac{n}{2}\right) \mathfrak{C}_{\alpha(n)}
$$

Here we give the most general expressions for $\mathfrak{C}_{\alpha(n)}$ for $n=2,3,4,5$. They are:

$$
\begin{aligned}
& \mathfrak{C}_{\alpha(2)}=\frac{1}{2}\left(2 \nabla_{\left(\alpha_{1}\right.}{ }^{\beta} h_{\left.\alpha_{2}\right) \beta}\right) \\
& \mathfrak{C}_{\alpha(3)}=\frac{1}{2^{2}}\left(3 \nabla_{\left(\alpha_{1}\right.}{ }^{\beta_{1}} \nabla_{\alpha_{2}}{ }^{\beta_{2}} h_{\left.\alpha_{3}\right) \beta(2)}+\square_{c} h_{\alpha(3)}\right), \\
& \mathfrak{C}_{\alpha(4)}=\frac{1}{2^{3}}\left(4 \nabla_{\left(\alpha_{1}\right.}{ }^{\beta_{1}} \nabla_{\alpha_{2}}{ }^{\beta_{2}} \nabla_{\alpha_{3}}{ }^{\beta_{3}} h_{\left.\alpha_{4}\right) \beta(3)}+4 \square_{c} \nabla_{\left(\alpha_{1}\right.}{ }^{\beta} h_{\left.\alpha_{2} \alpha_{3} \alpha_{4}\right) \beta}+a_{0} W_{\left(\alpha_{1} \alpha_{2}\right.}{ }^{\beta(2)} h_{\left.\alpha_{3} \alpha_{4}\right) \beta(2)}\right),
\end{aligned}
$$




$$
\begin{aligned}
\mathfrak{C}_{\alpha(5)}=\frac{1}{2^{4}}( & 5 \nabla_{\left(\alpha_{1}\right.}^{\beta_{1}} \nabla_{\alpha_{2}}^{\beta_{2}} \nabla_{\alpha_{3}}{ }^{\beta_{3}} \nabla_{\alpha_{4}}{ }^{\beta_{4}} h_{\left.\alpha_{5}\right) \beta(4)}+10 \square_{c} \nabla_{\left(\alpha_{1}\right.}{ }^{\beta_{1}} \nabla_{\alpha_{2}}{ }^{\beta_{2}} h_{\left.\alpha_{3} \alpha_{4} \alpha_{5}\right) \beta(2)}+\left(\square_{c}\right)^{2} h_{\alpha(5)} \\
& +\left(\frac{745}{16}-\frac{1}{2} a_{1}+\frac{5}{2} a_{2}+3 a_{3}\right) W^{\beta_{1} \beta_{2}}\left(\alpha_{1} \alpha_{2} \nabla_{\alpha_{3}}{ }^{\beta_{3}} h_{\left.\alpha_{4} \alpha_{5}\right) \beta(3)}\right. \\
& +\left(\frac{564}{48}-\frac{1}{2} a_{1}+\frac{1}{2} a_{2}+a_{3}\right) W_{\delta\left(\alpha_{1} \alpha_{2}\right.}{ }^{\beta_{1}} \nabla^{\delta \beta_{2}} h_{\left.\alpha_{3} \alpha_{4} \alpha_{5}\right) \beta(2)} \\
& +\left(-40+\frac{1}{2} a_{1}-\frac{1}{2} a_{2}-a_{3}\right) W^{\beta(3)}{ }_{\left(\alpha_{1}\right.} \nabla_{\alpha_{2} \alpha_{3}} h_{\left.\alpha_{4} \alpha_{5}\right) \beta(3)} \\
& +a_{1} \nabla^{\delta \beta_{1}} W^{\beta_{2}} \delta\left(\alpha_{1} \alpha_{2} h_{\left.\alpha_{3} \alpha_{4} \alpha_{5}\right) \beta(2)}+a_{2} \nabla_{\left(\alpha_{1}\right.}{ }^{\beta_{1}} W_{\alpha_{2} \alpha_{3}}^{\beta_{2} \beta_{3}} h_{\left.\alpha_{4} \alpha_{5}\right) \beta(3)}\right. \\
& \left.+a_{3} \nabla_{\left(\alpha_{1} \alpha_{2}\right.} W_{\alpha_{3}}^{\beta(3)} h_{\left.\alpha_{4} \alpha_{5}\right) \beta(3)}\right),
\end{aligned}
$$

where $\square_{c}=\nabla^{a} \nabla_{a}$ is the conformal d'Alembertian and the $a_{i}$ are arbitrary constants.

In a general curved background, for $n \geq 4$ the requirements outlined above do not determine $\mathfrak{C}_{\alpha(n)}$ uniquely, since we can always add appropriate terms involving $W_{\alpha(4)}$. However, in the $n=4$ case, one may fix the constant to $a_{0}=4$ by explicitly linearising $W_{\alpha(4)}$ around an arbitrary background as in (2.62b).

The higher-spin Cotton tensor is generally not gauge invariant and the aforementioned ambiguity associated with its definition cannot rectify this. From the expressions (3.5) it is evident that as $n$ increases this approach will become exceedingly difficult and the ambiguity will worsen. However, an attractive feature of this formulation occurs when the spacetime under consideration is conformally flat,

$$
W_{\alpha(4)}=0,
$$

and therefore the conformal covariant derivatives commute (2.50). Consequently, this ambiguity is eliminated and as we will now show, the unique expression, up to an overall normalisation, for the spin- $\frac{n}{2}$ Cotton tensor is

$$
\mathfrak{C}_{\alpha(n)}=\frac{1}{2^{n-1}} \sum_{j=0}^{\lceil n / 2\rceil-1}\left(\begin{array}{c}
n \\
2 j+1
\end{array}\right)\left(\square_{c}\right)^{j} \nabla_{\left(\alpha_{1}\right.}^{\beta_{1}} \ldots \nabla_{\alpha_{n-2 j-1}}^{\beta_{n-2 j-1}} h_{\left.\alpha_{n-2 j} \ldots \alpha_{n}\right) \beta_{1} \ldots \beta_{n-2 j-1}} .
$$

Here $\lceil n / 2\rceil$ denotes the ceiling function and is equal to $s$ for $n=2 s$ and $s+1$ for $n=2 s+1$, with $s \geq 0$ an integer. In the flat limit, (3.7) reduces to the one derived in [18]. For even values of $n, n=2,4, \ldots$, the flat-space version of (3.7) is equivalent to the one originally obtained by Pope and Townsend [3].

It is clear that (3.7) is of the form $\mathfrak{C}_{\alpha(n)}=\mathcal{A} h_{\alpha(n)}$ and has Weyl weight equal to $(1+n / 2)$. It remains to show that it is primary. Since $\mathfrak{C}_{\alpha(n)}$ is a covariant field, it suffices to show that under a $K$-transformation we have $\delta_{K} \mathfrak{C}_{\alpha(n)}=-\frac{1}{2} \Lambda(K)^{\gamma(2)} K_{\gamma(2)} \mathfrak{C}_{\alpha(n)}=0$.

Using the algebra (3.1) it is possible to show, by induction on $j$, that the following two identities hold true

$$
\begin{array}{r}
\Lambda(K)^{\gamma(2)}\left[K_{\gamma(2)},\left(\square_{c}\right)^{j}\right]=\Lambda(K)^{\gamma(2)}\left\{2 j\left(\square_{c}\right)^{j-1} \nabla_{\gamma(2)}(2 \mathbb{D}+2 j-3)\right. \\
\left.-4 j\left(\square_{c}\right)^{j-1} \nabla_{\gamma_{1}}{ }^{\delta} M_{\gamma_{2} \delta}\right\},
\end{array}
$$




$$
\begin{aligned}
& \Lambda(K)^{\gamma(2)} {\left[K_{\gamma(2)}, \nabla_{\left(\alpha_{1}\right.}^{\beta_{1}} \ldots \nabla_{\alpha_{j}}{ }^{\beta_{j}}\right] \zeta_{\left.\alpha_{j+1} \ldots \alpha_{m}\right) \beta_{1} \ldots \beta_{j}} } \\
&=\Lambda(K)^{\gamma(2)}\left\{4 j \delta_{\gamma_{1}}{ }^{\beta_{1}} \varepsilon_{\gamma_{2}\left(\alpha_{1}\right.} \nabla_{\alpha_{2}}{ }^{\beta_{2}} \ldots \nabla_{\alpha_{j}}{ }^{\beta_{j}}(\mathbb{D}+j-1)\right. \\
& \quad-2 j \nabla_{\left(\alpha_{1}\right.}{ }^{\beta_{1}} \ldots \nabla_{\alpha_{j-1}}{ }^{\beta_{j-1}} \varepsilon_{\alpha_{j} \mid \gamma_{1}} M_{\gamma_{2} \mid}{ }^{\beta_{j}}-2 j \nabla_{\left(\alpha_{1}\right.}{ }^{\beta_{1}} \ldots \nabla_{\alpha_{j-1}}{ }^{\beta_{j-1}} \delta_{\mid \gamma_{1}}{ }^{\beta_{j}} M_{\gamma_{2} \mid \alpha_{j}} \\
&\left.\quad-j(j-1) \nabla_{\gamma(2)} \delta_{\left(\alpha_{1}\right.}{ }^{\beta_{1}} \delta_{\alpha_{2}}{ }^{\beta_{2}} \nabla_{\alpha_{3}}{ }^{\beta_{3}} \ldots \nabla_{\alpha_{j}}{ }^{\beta_{j}}\right\} \zeta_{\left.\alpha_{j+1} \ldots \alpha_{m}\right) \beta_{1} \ldots \beta_{j}}
\end{aligned}
$$

where $\zeta_{\alpha(m)}$ is an arbitrary primary field. Therefore, under a special conformal transformation we have

$$
\begin{aligned}
& -2^{n} \delta_{K} \mathfrak{C}_{\alpha(n)}=\Lambda(K)^{\gamma(2)} \sum_{j=0}^{\lceil n / 2\rceil-1}\left(\begin{array}{c}
n \\
2 j+1
\end{array}\right)\left\{\left[K_{\gamma(2)},\left(\square_{c}\right)^{j}\right] \nabla_{\left(\alpha_{1}\right.}{ }^{\beta_{1}} \ldots \nabla_{\alpha_{n-2 j-1}} \beta_{n-2 j-1}\right. \\
& \left.+\left(\square_{c}\right)^{j}\left[K_{\gamma(2)}, \nabla_{\left(\alpha_{1}\right.}^{\beta_{1}} \ldots \nabla_{\alpha_{n-2 j-1}}^{\beta_{n-2 j-1}}\right]\right\} h_{\left.\alpha_{n-2 j} \ldots \alpha_{n}\right) \beta_{1} \ldots \beta_{n-2 j-1}} \\
& =\Lambda(K)^{\gamma(2)} \sum_{j=0}^{\lceil n / 2\rceil-1}\left(\begin{array}{c}
n \\
2 j+1
\end{array}\right) \\
& \times\left\{2 j ( 2 j + 1 ) ( \square _ { c } ) ^ { j - 1 } \left[\nabla_{\gamma(2)} \nabla_{\left(\alpha_{1}\right.}^{\beta_{1}} \ldots \nabla_{\alpha_{n-2 j-1}}^{\beta_{n-2 j-1}} h_{\left.\alpha_{n-2 j} \ldots \alpha_{n}\right) \beta_{1} \ldots \beta_{n-2 j-1}}\right.\right. \\
& \left.-2 \nabla_{\gamma_{1}\left(\alpha_{1}\right.} \nabla_{\alpha_{2}}{ }^{\beta_{2}} \ldots \nabla_{\alpha_{n-2 j}} \beta_{n-2 j} h_{\left.\alpha_{n-2 j+1} \ldots \alpha_{n}\right) \beta_{2} \ldots \beta_{n-2 j} \gamma_{2}}\right]-(n-2 j-1)(n-2 j-2) \\
& \times\left(\square_{c}\right)^{j}\left[\nabla_{\gamma(2)} \nabla_{\left(\alpha_{1}\right.}^{\beta_{1}} \ldots \nabla_{\alpha_{n-2 j-3}}^{\beta_{n-2 j-3}} h_{\left.\alpha_{n-2 j-2} \ldots \alpha_{n}\right) \beta_{1} \ldots \beta_{n-2 j-3}}\right. \\
& \left.\left.-2 \nabla_{\gamma_{1}\left(\alpha_{1}\right.} \nabla_{\alpha_{2}}{ }^{\beta_{2}} \ldots \nabla_{\alpha_{n-2 j-2}}^{\beta_{n-2 j-2}} h_{\left.\alpha_{n-2 j-1} \ldots \alpha_{n}\right) \beta_{2} \ldots \beta_{n-2 j-2} \gamma_{2}}\right]\right\}=0 .
\end{aligned}
$$

In the last line we have used the fact that the second and third terms vanish for $j=\lfloor n / 2\rfloor$ and the first and last terms vanish for $j=0$ to shift the summation variable. This shows that in any conformally flat space (3.7) is the unique tensor satisfying the properties listed at the beginning of this section.

\subsection{CHS actions}

For every conformally flat spacetime, the tensor (3.7) has the following properties:

1. $\mathfrak{C}_{\alpha(n)}$ is conserved,

$$
\nabla^{\beta(2)} \mathfrak{C}_{\alpha(n-2) \beta(2)}=0
$$

2. $\mathfrak{C}_{\alpha(n)}$ is invariant under the gauge transformations (3.3),

$$
\delta_{\xi} h_{\alpha(n)}=\nabla_{\left(\alpha_{1} \alpha_{2}\right.} \xi_{\left.\alpha_{3} \ldots \alpha_{n}\right)} \quad \Longrightarrow \quad \delta_{\xi} \mathfrak{C}_{\alpha(n)}=0
$$

In a general curved space, $\mathfrak{C}_{\alpha(n)}$ must reduce to the expression (3.7) in the conformally flat limit. Therefore, in such spaces the right hand side of eq. (3.7) constitutes the skeleton 
of $\mathfrak{C}_{\alpha(n)}$. It immediately follows that its divergence and gauge variation under (3.3) are proportional to terms involving $W_{\alpha(4)}$ and its covariant derivatives,

$$
\nabla^{\beta(2)} \mathfrak{C}_{\alpha(n-2) \beta(2)}=\mathcal{O}\left(W_{\alpha(4)}\right), \quad \delta_{\xi} \mathfrak{C}_{\alpha(n)}=\mathcal{O}\left(W_{\alpha(4)}\right) .
$$

The properties listed in eq. (3.4) ensure that the linearised conformal higher-spin action

$$
S_{\mathrm{CS}}^{(n)}[h]=-\frac{\mathrm{i}^{n}}{2^{\lfloor n / 2\rfloor}} \int \mathrm{d}^{3} x e h^{\alpha(n)} \mathfrak{C}_{\alpha(n)}(h),
$$

is invariant under the conformal gauge group $\mathcal{G}$. Furthermore, by virtue of (3.9) and (3.10), in any conformally flat space (3.12) is invariant under the gauge transformations (3.3),

$$
W_{a b}=0 \quad \Longrightarrow \quad \delta_{\xi} S_{\mathrm{CS}}^{(n)}=0 .
$$

Upon degauging and setting $n=4$, the model (3.12) coincides with the action for linearised conformal gravity given by eq. (2.63).

We would like to point out the following interesting realisation of $\mathfrak{C}_{\alpha(n)}$ in terms of the projection operators

$$
\Pi_{\alpha}^{( \pm) \beta}=\frac{1}{2}\left(\delta_{\alpha}^{\beta} \pm \frac{\nabla_{\alpha}^{\beta}}{\sqrt{\square_{c}}}\right), \quad \Pi_{\alpha(n)}^{( \pm n) \beta(n)}=\Pi_{\left(\alpha_{1}\right.}^{( \pm) \beta_{1}} \ldots \Pi_{\left.\alpha_{n}\right)}^{( \pm)} \beta_{n},
$$

which are obtained by extending the flat-space results of [53]. Then one can express the higher-spin Cotton tensor as

$$
\mathfrak{C}_{\alpha(n)}=\left(\square_{c}\right)^{(n-1) / 2}\left(\Pi^{(n)}-(-1)^{n} \Pi^{(-n)}\right) h_{\alpha(n)} .
$$

We may use the expressions (3.15) to rewrite the action (3.12) in terms of the projection operators.

\subsection{Generalised CHS models}

As an extension of the previous constructions, we now consider a conformal higher-spin gauge field $h_{\alpha(n)}^{(l)}$ which is primary and defined modulo gauge transformations of depth $l,{ }^{7}$

$$
\delta_{\xi} h_{\alpha(n)}^{(l)}=\nabla_{\left(\alpha_{1} \alpha_{2}\right.} \cdots \nabla_{\alpha_{2 l-1} \alpha_{2 l}} \xi_{\left.\alpha_{2 l+1} \ldots \alpha_{n}\right)}
$$

where $l$ is some integer $1 \leq l \leq\left\lfloor\frac{n}{2}\right\rfloor$. We also require that the gauge parameter $\xi_{\alpha(n-2 l)}$ be primary, after which one can show, using the identity

$$
\begin{aligned}
\Lambda(K)^{\gamma(2)}\left[K_{\gamma(2)}, \nabla_{\left(\alpha_{1} \alpha_{2}\right.} \ldots \nabla_{\alpha_{2 l-1} \alpha_{2 l}}\right] \xi_{\left.\alpha_{2 l+1} \ldots \alpha_{n}\right)} & \\
=\Lambda(K)^{\gamma(2)}\{ & 4 l \varepsilon_{\left(\alpha_{1} \mid \gamma_{1}\right.} \varepsilon_{\gamma_{2} \mid \alpha_{2}} \nabla_{\alpha_{3} \alpha_{4}} \ldots \nabla_{\alpha_{2 l-1} \alpha_{2 l}}(\mathbb{D}+l-1) \\
& \left.\quad 4 l \nabla_{\left(\alpha_{1} \alpha_{2}\right.} \ldots \nabla_{\alpha_{2 l-3} \alpha_{2 l-2}} \varepsilon_{\alpha_{2 l-1} \mid \gamma_{1}} M_{\gamma_{2} \mid \alpha_{2 l}}\right\} \xi_{\left.\alpha_{2 l+1} \ldots \alpha_{n}\right)},
\end{aligned}
$$

\footnotetext{
${ }^{7}$ Such gauge transformations occur in the description of partially massless fields in diverse dimensions [54-69].
} 
that the dimension of $h_{\alpha(n)}$ is fixed to $(l+1)-\frac{n}{2}$. The conformal properties of $h_{\alpha(n)}^{(l)}$ may then be summarised by

$$
K_{\beta(2)} h_{\alpha(n)}^{(l)}=0, \quad \mathbb{D} h_{\alpha(n)}^{(l)}=\left(l+1-\frac{n}{2}\right) h_{\alpha(n)}^{(l)} .
$$

As was done earlier in the case $l=1$, from $h_{\alpha(n)}^{(l)}$ we may construct a generalised higher-spin Cotton tensor $\mathfrak{C}_{\alpha(n)}^{(l)}(h)$ that possesses the following conformal properties,

$$
K_{\beta(2)} \mathfrak{C}_{\alpha(n)}^{(l)}=0, \quad \mathbb{D} \mathfrak{C}_{\alpha(n)}^{(l)}=\left(2-l+\frac{n}{2}\right) \mathfrak{C}_{\alpha(n)}^{(l)} .
$$

In any conformally flat space, the properties (3.19) determine $\mathfrak{C}_{\alpha(n)}^{(l)}$ uniquely, up to an overall normalisation, to be

$$
\begin{aligned}
\mathfrak{C}_{\alpha(n)}^{(l)}= & \frac{1}{2^{n-2 l+1}} \sum_{j=l-1}^{\lceil n / 2\rceil-1}\left(\begin{array}{c}
n \\
2 j+1
\end{array}\right)\left(\begin{array}{c}
j \\
l-1
\end{array}\right)\left(\square_{c}\right)^{j-l+1} \\
& \times \nabla_{\left(\alpha_{1}\right.} \beta_{1} \ldots \nabla_{\alpha_{n-2 j-1}} \beta_{n-2 j-1} h_{\left.\alpha_{n-2 j} \ldots \alpha_{n}\right) \beta_{1} \ldots \beta_{n-2 j-1}}^{(l)} .
\end{aligned}
$$

To derive (3.20) we have made use of the identities (3.8). The properties (3.18) and (3.19) mean that the generalised higher-spin Chern-Simons action,

$$
S_{\mathrm{CS}}^{(n, l)}[h]=-\frac{\mathrm{i}^{n}}{2^{\lfloor n / 2\rfloor}} \int \mathrm{d}^{3} x e h_{(l)}^{\alpha(n)} \mathfrak{C}_{\alpha(n)}^{(l)}(h),
$$

is invariant under the conformal gauge group $\mathcal{G}$. Moreover, in any conformally flat space the generalised higher-spin Cotton tensor possesses the following important properties:

1. $\mathfrak{C}_{\alpha(n)}^{(l)}$ is partially conserved,

$$
\nabla^{\beta_{1} \beta_{2}} \cdots \nabla^{\beta_{2 l-1} \beta_{2 l}} \mathfrak{C}_{\alpha(n-2 l) \beta(2 l)}^{(l)}=0 .
$$

2. $\mathfrak{C}_{\alpha(n)}^{(l)}$ is gauge invariant,

$$
\delta_{\xi} h_{\alpha(n)}^{(l)}=\nabla_{\left(\alpha_{1} \alpha_{2}\right.} \cdots \nabla_{\alpha_{2 l-1} \alpha_{2 l}} \xi_{\left.\alpha_{2 l+1} \ldots \alpha_{n}\right)} \quad \Longrightarrow \quad \delta_{\xi} \mathfrak{C}_{\alpha(n)}^{(l)}=0 .
$$

As a consequence, the action (3.21) is also gauge invariant,

$$
W_{a b}=0 \quad \Longrightarrow \quad \delta_{\xi} S_{\mathrm{CS}}^{(n, l)}=0 .
$$

The proofs for the properties (3.22) are non-trivial and are given in appendix C. ${ }^{8}$

An interesting question to ask is the following. For a given spin, which values of $l$ yield first and second-order Lagrangians in the action (3.21)? To answer this question, we observe that the number of covariant derivatives in $(3.21)$ is $(n-2 l+1)$ so that $l=\frac{1}{2} n$ and $l=\frac{1}{2}(n-1)$, respectively. Since $l$ must be an integer it immediately follows that first-order conformal models exist only for bosonic spin whilst second-order models must be fermionic. These models are said to have 'maximal depth' since $l$ assumes its maximal value of $l=\left\lfloor\frac{n}{2}\right\rfloor$. Our conclusions regarding second-order models are in agreement with those drawn long ago in [72].

\footnotetext{
${ }^{8}$ It would be of interest to apply the methods of $[70,71]$ to demonstrate that $(3.20)$ is the most general solution of the $l$-folded conservation equation (3.22a) in the case of Minkowski spacetime.
} 


\subsection{Degauging}

In the gauge (2.25), the spinor covariant derivative assumes the form

$$
\nabla_{\alpha(2)}=\mathcal{D}_{\alpha(2)}-\frac{1}{4} P_{\alpha(2),}{ }^{\beta(2)} K_{\beta(2)} .
$$

We may decompose $P_{\alpha(2), \beta(2)}$ into irreducible components as

$$
P_{\alpha(2), \beta(2)}=R_{\alpha(2) \beta(2)}+\frac{1}{6} \varepsilon_{\alpha_{1}\left(\beta_{1}\right.} \varepsilon_{\left.\beta_{2}\right) \alpha_{2}} R,
$$

where $R_{\alpha(4)}$ is the background traceless Ricci tensor. The goal is then to replace all occurrences of $\nabla_{\alpha(2)}$ with (3.24) and use the algebra (3.1) to eliminate $K_{a}$. In general, this is a difficult technical problem, particularly for higher-derivative tensors such as (3.7).

As an example, in $\mathrm{AdS}_{3}$ the conformal covariant derivative is

$$
\nabla_{\alpha(2)}=\mathcal{D}_{\alpha(2)}-\mathcal{S}^{2} K_{\alpha(2)}
$$

whilst the conformal d'Alembertian is

$$
\square_{c}=\square-6 \mathcal{S}^{2} \mathbb{D}+\mathcal{S}^{2} \mathcal{D}^{\alpha(2)} K_{\alpha(2)}-\frac{1}{2} \mathcal{S}^{4} K^{\alpha(2)} K_{\alpha(2)} .
$$

Here and in (3.26), the parameter $\mathcal{S}$ is related to the AdS scalar curvature through $R=$ $-24 \mathcal{S}^{2}$. Making use of the above relations, one may show that the degauged version of (3.7), for small $n$, coincides with the ones given in [20] (up to conventions). However, for general $n$ we were not able to obtain a closed form expression.

It should be pointed out that various aspects of the bosonic higher-spin Cotton tensors were studied in $[73,74]$.

\section{Conformal higher-spin models in four dimensions}

In this section we work in four dimensions, $D=4$, and make use of the two-component spinor notation and conventions in [33]. It is convenient to replace the Lorentz generators $M_{a b}=-M_{b a}$ with operators carrying spinor indices, $M_{\alpha \beta}=M_{\beta \alpha}$ and $\bar{M}_{\dot{\alpha} \dot{\beta}}=\bar{M}_{\dot{\beta} \dot{\alpha}}$, which are defined as

$$
\begin{aligned}
M_{\alpha \beta} & =\frac{1}{2}\left(\sigma^{a b}\right)_{\alpha \beta} M_{a b}, \quad \bar{M}_{\dot{\alpha} \dot{\beta}}=-\frac{1}{2}\left(\tilde{\sigma}^{a b}\right)_{\dot{\alpha} \dot{\beta}} M_{a b}, \\
M^{a b} & =\left(\sigma^{a b}\right)_{\alpha \beta} M^{\alpha \beta}-\left(\tilde{\sigma}^{a b}\right)_{\dot{\alpha} \dot{\beta}} \bar{M}^{\dot{\alpha} \dot{\beta}}
\end{aligned}
$$

and act only on undotted and dotted indices, respectively.

In the two-component spinor notation eq. (2.40) takes the form

$$
\begin{aligned}
{\left[\nabla_{\alpha \dot{\alpha}}, \nabla_{\beta \dot{\beta}}\right]=} & -\left(\varepsilon_{\dot{\alpha} \dot{\beta}} C_{\alpha \beta \gamma \delta} M^{\gamma \delta}+\varepsilon_{\alpha \beta} \bar{C}_{\dot{\alpha} \dot{\beta} \dot{\gamma} \dot{\delta}} \bar{M}^{\dot{\gamma} \dot{\delta}}\right) \\
& -\frac{1}{4}\left(\varepsilon_{\dot{\alpha} \dot{\beta}} \nabla^{\delta \dot{\gamma}} C_{\alpha \beta \delta}{ }^{\gamma}+\varepsilon_{\alpha \beta} \nabla^{\gamma \dot{\delta}} \bar{C}_{\dot{\alpha} \dot{\beta} \dot{\delta}} \dot{\gamma}\right) K_{\gamma \dot{\gamma}}
\end{aligned}
$$


whilst the commutation relations of the remaining generators with $\nabla_{\beta \dot{\beta}}$ are given by

$$
\begin{aligned}
{\left[M_{\alpha \gamma}, \nabla_{\beta \dot{\beta}}\right] } & =\varepsilon_{\beta(\alpha} \nabla_{\gamma) \dot{\beta}}, \quad\left[\bar{M}_{\dot{\alpha} \dot{\gamma}}, \nabla_{\beta \dot{\beta}}\right]=\varepsilon_{\dot{\beta}(\dot{\alpha}} \nabla_{\beta \dot{\gamma})}, \\
{\left[\mathbb{D}, \nabla_{\beta \dot{\beta}}\right] } & =\nabla_{\beta \dot{\beta}}, \\
{\left[K_{\alpha \dot{\alpha}}, \nabla_{\beta \dot{\beta}}\right] } & =4 \varepsilon_{\dot{\alpha} \dot{\beta}} M_{\alpha \beta}+4 \varepsilon_{\alpha \beta} \bar{M}_{\dot{\alpha} \dot{\beta}}-4 \varepsilon_{\alpha \beta} \varepsilon_{\dot{\alpha} \dot{\beta}} \mathbb{D} .
\end{aligned}
$$

In eq. (4.2a) the self-dual and anti self-dual Weyl tensors $C_{\alpha \beta \gamma \delta}$ and $\bar{C}_{\dot{\alpha} \dot{\beta} \dot{\gamma} \dot{\delta}}$ are related to $C_{a b c d}$ as follows

$$
\begin{aligned}
C_{\alpha \beta \gamma \delta} & =\frac{1}{2}\left(\sigma^{a b}\right)_{\alpha \beta}\left(\sigma^{c d}\right)_{\gamma \delta} C_{a b c d}=C_{(\alpha \beta \gamma \delta)}, \\
\bar{C}_{\dot{\alpha} \dot{\beta} \dot{\gamma} \dot{\delta}} & =\frac{1}{2}\left(\tilde{\sigma}^{a b}\right)_{\dot{\alpha} \dot{\beta}}\left(\tilde{\sigma}^{c d}\right)_{\dot{\gamma} \dot{\delta}} C_{a b c d}=\bar{C}_{(\dot{\alpha} \dot{\beta} \dot{\gamma} \dot{\delta})}, \\
C_{\alpha \beta \gamma \delta \dot{\alpha} \dot{\beta} \dot{\gamma} \dot{\delta}} & =\left(\sigma^{a}\right)_{\alpha \dot{\alpha}}\left(\sigma^{b}\right)_{\beta \dot{\beta}}\left(\sigma^{c}\right)_{\gamma \dot{\gamma}}\left(\sigma^{d}\right)_{\delta \dot{\delta}} C_{a b c d}=2 \varepsilon_{\dot{\alpha} \dot{\beta}} \varepsilon_{\dot{\gamma} \dot{\delta}} C_{\alpha \beta \gamma \delta}+2 \varepsilon_{\alpha \beta} \varepsilon_{\gamma \delta} \bar{C}_{\dot{\alpha} \dot{\beta} \dot{\gamma} \dot{\delta}} .
\end{aligned}
$$

\subsection{CHS prepotentials and field strengths}

We introduce conformal higher-spin gauge fields by generalising the constructions in [17] and earlier works $[4,5]$. Given two positive integers $m$ and $n$, a conformal higher-spin gauge prepotential $\phi_{\alpha(m) \dot{\alpha}(n)}$ is a primary field defined modulo gauge transformations

$$
\delta_{\lambda} \phi_{\alpha(m) \dot{\alpha}(n)}=\nabla_{\left(\alpha _ { 1 } \left(\dot{\alpha}_{1}\right.\right.} \lambda_{\left.\left.\alpha_{2} \ldots \alpha_{m}\right) \dot{\alpha}_{2} \ldots \dot{\alpha}_{n}\right)},
$$

where the gauge parameter $\lambda_{\alpha(m-1) \dot{\alpha}(n-1)}$ is also assumed to be primary. This gauge freedom uniquely fixes the conformal dimension of the gauge field,

$$
K_{\beta \dot{\beta}} \phi_{\alpha(m) \dot{\alpha}(n)}=0, \quad \mathbb{D} \phi_{\alpha(m) \dot{\alpha}(n)}=\left(2-\frac{1}{2}(m+n)\right) \phi_{\alpha(m) \dot{\alpha}(n)} .
$$

In the $m \neq n$ case, the gauge prepotential $\phi_{\alpha(m) \dot{\alpha}(n)}$ and its gauge parameter $\lambda_{\alpha(m-1) \dot{\alpha}(n-1)}$ are complex.

From $\phi_{\alpha(m) \dot{\alpha}(n)}$ one may construct two descendants (and their conjugates) to which we refer as higher-spin Weyl tensors and denote by $\hat{\mathfrak{C}}_{\alpha(m+n)}$ and $\check{\mathfrak{C}}_{\alpha(m+n)}$. They possess the following key properties:

1. $\hat{\mathfrak{C}}_{\alpha(m+n)}$ and $\check{\mathfrak{C}}_{\alpha(m+n)}$ are of the form $\hat{\mathcal{A}} \phi_{\alpha(m) \dot{\alpha}(n)}$ and $\check{\mathcal{A}} \bar{\phi}_{\alpha(n) \dot{\alpha}(m)}$, respectively. Here $\hat{\mathcal{A}}$ and $\breve{\mathcal{A}}$ are linear differential operators involving the covariant derivatives, the Weyl tensor $C_{a b c d}$, and its covariant derivatives.

2. Both $\hat{\mathfrak{C}}_{\alpha(m+n)}$ and $\check{\mathfrak{C}}_{\alpha(m+n)}$ are primary fields of dimension $2-\frac{1}{2}(m-n)$ and $2-$ $\frac{1}{2}(n-m)$, respectively,

$$
\begin{array}{lll}
K_{\beta \dot{\beta}} \hat{\mathfrak{C}}_{\alpha(m+n)}=0, & \mathbb{D} \hat{\mathfrak{C}}_{\alpha(m+n)}=\left(2-\frac{1}{2}(m-n)\right) \hat{\mathfrak{C}}_{\alpha(m+n)}, \\
K_{\beta \dot{\beta}} \check{\mathfrak{C}}_{\alpha(m+n)}=0, & \mathbb{D} \check{\mathfrak{C}}_{\alpha(m+n)}=\left(2-\frac{1}{2}(n-m)\right) \check{\mathfrak{C}}_{\alpha(m+n)} .
\end{array}
$$

Strictly speaking, we should use a more detailed notation for $\hat{\mathfrak{C}}_{\alpha(m+n)}$ and $\check{\mathfrak{C}}_{\alpha(m+n)}$ that would explicitly indicate the values of $m$ and $n$, say $\mathfrak{C}_{\alpha(m+n)}^{(m, n)}$ instead of $\hat{\mathfrak{C}}_{\alpha(m+n)}$. This 
is because there are several choices for $m$ and $n$ such that $m+n=$ const. However, in the hope that no confusion will arise we do not use such a cumbersome notation.

In some respect the $4 D$ case is simpler than its $3 D$ counterpart. For instance, in a general curved space the higher-spin Weyl tensors take the form

$$
\begin{aligned}
& \hat{\mathfrak{C}}_{\alpha(m+n)}=\nabla_{\left(\alpha_{1}\right.} \dot{\beta}_{1} \ldots \nabla_{\alpha_{n}}{ }^{\dot{\beta}_{n}} \phi_{\left.\alpha_{n+1} \ldots \alpha_{n+m}\right) \dot{\beta}_{1} \ldots \dot{\beta}_{n}}, \\
& \check{\mathfrak{C}}_{\alpha(m+n)}=\nabla_{\left(\alpha_{1}\right.} \dot{\beta}_{1} \ldots \nabla_{\alpha_{m}} \dot{\beta}_{m} \bar{\phi}_{\left.\alpha_{m+1} \ldots \alpha_{m+n}\right) \dot{\beta}_{1} \ldots \dot{\beta}_{m}} .
\end{aligned}
$$

It is clear that both (4.7a) and (4.7b) are of the form specified in property one, and also that they have the correct Weyl weights as prescribed in property two. We now show that they are primary.

Using the algebra (4.2) it is possible to prove, via induction on $j$, that the following identity holds

$$
\begin{aligned}
& {\left[K_{\gamma \dot{\gamma}}, \nabla_{\left(\alpha_{1}\right.} \dot{\beta}_{1} \ldots \nabla_{\alpha_{j}} \dot{\beta}_{j}\right] \zeta_{\left.\alpha_{j+1} \ldots \alpha_{j+i}\right) \dot{\beta}_{1} \ldots \dot{\beta}_{j}}} \\
& =-\left\{4 j \nabla_{\left(\alpha_{1}\right.} \dot{\beta}_{1} \ldots \nabla_{\alpha_{j-1}} \dot{\beta}_{j-1} \varepsilon_{\alpha_{j} \mid \gamma} \delta_{\dot{\gamma} \mid} \dot{\beta}_{j} \mathbb{D}+4 j(j-1) \nabla_{\left(\alpha_{1}\right.} \dot{\beta}_{1} \ldots \nabla_{\alpha_{j-1}} \dot{\beta}_{j-1} \varepsilon_{\alpha_{j} \mid \gamma} \delta_{\dot{\gamma} \mid} \dot{\beta}_{j}\right. \\
& \quad+4 j \nabla_{\left(\alpha_{1}\right.} \dot{\beta}_{1} \ldots \nabla_{\alpha_{j-1}} \dot{\beta}_{j-1} M_{\alpha_{j} \mid \gamma} \delta_{\dot{\gamma} \mid} \dot{\beta}_{j} \\
& \left.\quad+4 j \nabla_{\left(\alpha_{1}\right.} \dot{\beta}_{1} \ldots \nabla_{\alpha_{j-1}} \dot{\beta}_{j-1} \varepsilon_{\alpha_{j} \mid \gamma} \bar{M}_{\dot{\gamma} \mid} \dot{\beta}_{j}\right\} \zeta_{\left.\alpha_{j+1} \ldots \alpha_{j+i}\right) \dot{\beta}_{1} \ldots \dot{\beta}_{j}}
\end{aligned}
$$

where $\zeta_{\alpha(i) \dot{\alpha}(j)}$ is an arbitrary primary field. When the field in (4.8) is restricted to carry the Weyl weight specified in (4.5), upon setting $j=n$ and $i=m$ and evaluating, one finds that the right hand side vanishes. This demonstrates that $\hat{\mathfrak{C}}_{\alpha(m+n)}$ is primary. A similar argument holds for $\check{\mathfrak{C}}_{\alpha(m+n)}$.

In a general curved space, one may construct the following primary descendants from the higher-spin Weyl tensors,

$$
\begin{aligned}
& \hat{\mathfrak{B}}_{\alpha(n) \dot{\beta}(m)}=\nabla_{\left(\dot{\beta}_{1}\right.} \gamma_{1} \ldots \nabla_{\left.\dot{\beta}_{m}\right)} \gamma_{m} \hat{\mathfrak{C}}_{\alpha_{1} \ldots \alpha_{n} \gamma_{1} \ldots \gamma_{m}}, \\
& \check{\mathfrak{B}}_{\alpha(m) \dot{\beta}(n)}=\nabla_{\left(\dot{\beta}_{1}\right.}{ }^{\gamma_{1}} \ldots \nabla_{\left.\dot{\beta}_{n}\right)}{ }^{\gamma_{n}} \check{\mathfrak{C}}_{\alpha_{1} \ldots \alpha_{m} \gamma_{1} \ldots \gamma_{n}} .
\end{aligned}
$$

Both (4.9a) and (4.9b) have Weyl weight $2+\frac{1}{2}(m+n)$. The proof that they are primary is similar to that of the higher-spin Weyl tensors and makes use of the identity (4.8) and the properties (4.6). The primary fields (4.9a) and (4.9b) originate from two alternative expressions for one and the same conformal invariant

$$
\int \mathrm{d}^{4} x e \hat{\mathfrak{C}}^{\alpha(m+n)} \check{\mathfrak{C}}_{\alpha(m+n)}=\int \mathrm{d}^{4} x e \phi^{\alpha(m) \dot{\beta}(n)} \check{\mathfrak{B}}_{\alpha(m) \dot{\beta}(n)}=\int \mathrm{d}^{4} x e \bar{\phi}^{\alpha(n) \dot{\beta}(m)} \hat{\mathfrak{B}}_{\alpha(n) \dot{\beta}(m)} .
$$

The derivation of (4.10) is given in appendix D. We will call $\hat{\mathfrak{B}}_{\alpha(n) \dot{\beta}(m)}$ and $\check{\mathfrak{B}}_{\alpha(m) \dot{\beta}(n)}$ (linearised) higher-spin Bach tensors. 


\subsection{CHS actions}

The expressions (4.7a) and (4.7b) are determined uniquely, modulo terms involving the background Weyl tensor $C_{a b c d}$, by the key properties listed earlier. However, when the background spacetime is conformally flat, (4.7a) and (4.7b) are the unique higher-spin Weyl tensors. By virtue of the commutator (4.2a) they are also invariant under gauge transformations (4.4),

$$
C_{a b c d}=0 \quad \Longrightarrow \quad \delta_{\lambda} \hat{\mathfrak{C}}_{\alpha(m+n)}=\delta_{\lambda} \check{\mathfrak{C}}_{\alpha(m+n)}=0 .
$$

Since the commutator of covariant derivatives is proportional to the Weyl tensor, it follows that the gauge variation of (4.7a) and (4.7b) under (4.4) in an arbitrarily curved space is proportional to the Weyl tensor and its covariant derivatives,

$$
\delta_{\lambda} \hat{\mathfrak{C}}_{\alpha(m+n)}=\mathcal{O}\left(C_{a b c d}\right), \quad \delta_{\lambda} \check{\mathfrak{C}}_{\alpha(m+n)}=\mathcal{O}\left(C_{a b c d}\right)
$$

As a consequence of the properties (4.6), the linearised conformal higher-spin action

$$
S_{\mathrm{CHS}}^{(m, n)}[\phi, \bar{\phi}]=\mathrm{i}^{m+n} \int \mathrm{d}^{4} x e \hat{\mathfrak{C}}^{\alpha(m+n)} \check{\mathfrak{C}}_{\alpha(m+n)}+\text { c.c. }
$$

is invariant under the gauge group $\mathcal{G}$. Additionally, by virtue of (4.11), in any conformally flat space it is also invariant under the gauge transformations (4.4),

$$
C_{a b c d}=0 \quad \Longrightarrow \quad \delta_{\lambda} S_{\mathrm{CHS}}^{(m, n)}=0 .
$$

In any conformally flat background the two terms in the right-hand side of (4.13) coincide because of the identity

$$
\mathrm{i}^{m+n+1} \int \mathrm{d}^{4} x e \hat{\mathfrak{C}}^{\alpha(m+n)} \check{\mathfrak{C}}_{\alpha(m+n)}+\text { c.c. } \approx 0 .
$$

In appendices $\mathrm{E}$ and $\mathrm{F}$ we discuss how the action (4.13) can be deformed to make it gauge invariant in Bach-flat backgrounds for low spin values.

When spacetime is conformally flat, the tensors (4.9) possess the following properties:

1. $\hat{\mathfrak{B}}_{\alpha(n) \dot{\beta}(m)}$ and $\check{\mathfrak{B}}_{\alpha(m) \dot{\beta}(n)}$ are invariant under the gauge transformations (4.4),

$$
\delta_{\lambda} \hat{\mathfrak{B}}_{\alpha(n) \dot{\beta}(m)}=\delta_{\lambda} \check{\mathfrak{B}}_{\alpha(m) \dot{\beta}(n)}=0 .
$$

2. $\hat{\mathfrak{B}}_{\alpha(n) \dot{\beta}(m)}$ and $\check{\mathfrak{B}}_{\alpha(m) \dot{\beta}(n)}$ are transverse,

$$
\nabla^{\gamma \dot{\gamma}} \hat{\mathfrak{B}}_{\gamma \alpha(n-1) \dot{\gamma} \dot{\beta}(m-1)}=\nabla^{\gamma \dot{\gamma}} \check{\mathfrak{B}}_{\gamma \alpha(m-1) \dot{\gamma} \dot{\beta}(n-1)}=0 .
$$

3. The complex conjugates of $\hat{\mathfrak{B}}_{\alpha(n) \dot{\beta}(m)}$ and $\check{\mathfrak{B}}_{\alpha(m) \dot{\beta}(n)}$ satisfy

$$
\overline{\hat{\mathfrak{B}}}_{\alpha(m) \dot{\beta}(n)}=\check{\mathfrak{B}}_{\alpha(m) \dot{\beta}(n)}, \quad \overline{\mathfrak{\mathfrak { B }}}_{\alpha(n) \dot{\beta}(m)}=\hat{\mathfrak{B}}_{\alpha(n) \dot{\beta}(m)} .
$$


The first two properties are obvious. The third property contains non-trivial information and when written out in its entirety reads

$$
\begin{aligned}
\nabla_{\left(\alpha_{1}\right.}{ }^{\dot{\gamma}_{1}} \ldots \nabla_{\left.\alpha_{m}\right)} \dot{\gamma}_{m} \overline{\hat{\mathfrak{C}}}_{\dot{\beta}_{1} \ldots \dot{\beta}_{n} \dot{\gamma}_{1} \ldots \dot{\gamma}_{m}}=\nabla_{\left(\dot{\beta}_{1}\right.}{ }^{\gamma_{1}} \ldots \nabla_{\left.\dot{\beta}_{n}\right)}{ }^{\gamma_{n} \check{\mathfrak{C}}_{\alpha_{1} \ldots \alpha_{m} \gamma_{1} \ldots \gamma_{n}}} \\
\nabla_{\left(\alpha_{1}\right.} \dot{\gamma}_{1} \ldots \nabla_{\left.\alpha_{n}\right)} \dot{\gamma}_{n} \overline{\mathfrak{C}}_{\dot{\beta}_{1} \ldots \dot{\beta}_{m} \dot{\gamma}_{1} \ldots \dot{\gamma}_{n}}=\nabla_{\left(\dot{\beta}_{1}\right.}{ }^{\gamma_{1}} \ldots \nabla_{\left.\dot{\beta}_{m}\right)}{ }^{\gamma_{m}} \hat{\mathfrak{C}}_{\alpha_{1} \ldots \alpha_{n} \gamma_{1} \ldots \gamma_{m}} .
\end{aligned}
$$

To prove (4.17a) one can assume, without loss of generality, that $m \geq n$. It may then be shown that both sides of the equality evaluate to

$$
\begin{aligned}
\frac{1}{(m+n) !} \sum_{j=0}^{n}\left(\begin{array}{c}
m \\
j
\end{array}\right)\left(\begin{array}{c}
n \\
j
\end{array}\right) m ! n !\left(\square_{c}\right)^{j} \nabla_{\left(\alpha_{1}\right.}^{\dot{\gamma}_{1}} \cdots & \nabla_{\alpha_{m-j}} \dot{\gamma}_{m-j} \nabla_{\left(\dot{\beta}_{1}\right.} \gamma_{1} \ldots \nabla_{\dot{\beta}_{n-j}} \gamma_{n-j} \\
& \times \bar{\phi}_{\left.\left.\alpha_{m-j+1} \ldots \alpha_{m}\right) \gamma_{1} \ldots \gamma_{n-j} \dot{\beta}_{n-j+1} \ldots \dot{\beta}_{n}\right) \dot{\gamma}_{1} \ldots \dot{\gamma}_{m-j}}
\end{aligned}
$$

The proof for $(4.17 \mathrm{~b})$ is similar.

It is instructive to introduce the spin projection operators $\Pi^{(m, n)}$ and $\Pi^{[m, n]}$ which are defined by their action on tensor fields,

$$
\begin{aligned}
\Pi^{(m, n)} \phi_{\alpha(m) \dot{\beta}(n)} & =\Delta_{\dot{\beta}_{1}}{ }^{\gamma_{1}} \cdots \Delta_{\dot{\beta}_{n}}{ }^{\gamma_{n}} \Delta_{\left(\gamma_{1}\right.} \dot{\gamma}_{1} \cdots \Delta_{\gamma_{n}}^{\dot{\gamma}_{n}} \phi_{\left.\alpha_{1} \ldots \alpha_{m}\right) \dot{\gamma}_{1} \ldots \dot{\gamma}_{n}}, \\
\Pi^{[m, n]} \phi_{\alpha(m) \dot{\beta}(n)} & =\Delta_{\alpha_{1}} \dot{\gamma}_{1} \cdots \Delta_{\alpha_{m}}{ }^{\dot{\gamma}_{m}} \Delta_{\left(\dot{\gamma}_{1}\right.}{ }^{\gamma_{1}} \cdots \Delta_{\dot{\gamma}_{m}}{ }^{\gamma_{m}} \phi_{\left.\gamma_{1} \ldots \gamma_{m} \dot{\beta}_{1} \ldots \dot{\beta}_{n}\right)} .
\end{aligned}
$$

Here we have made use of the involutive operator ${ }^{9}$

$$
\Delta_{\alpha} \dot{\beta}=\frac{\nabla_{\alpha}^{\dot{\beta}}}{\sqrt{\square_{c}}}, \quad \Delta_{\alpha}{ }^{\dot{\beta}} \Delta_{\dot{\beta}}{ }^{\beta}=\delta_{\alpha}{ }^{\beta}, \quad \Delta_{\dot{\alpha}}{ }^{\beta} \Delta_{\beta}{ }^{\dot{\beta}}=\delta_{\dot{\alpha}}^{\dot{\beta}}
$$

In a fashion similar to the proof of (4.17), it may be shown that both projection operators are equal to one another,

$$
\Pi^{(m, n)} \phi_{\alpha(m) \dot{\beta}(n)}=\Pi^{[m, n]} \phi_{\alpha(m) \dot{\beta}(n)} .
$$

In addition, they satisfy the following properties

$$
\begin{aligned}
\Pi^{(m, n)} \Pi^{(m, n)} & =\Pi^{(m, n)}, & \Pi^{[m, n]} \Pi^{[m, n]} & =\Pi^{[m, n} \\
\nabla^{\gamma \dot{\gamma}} \Pi^{(m, n)} \phi_{\gamma \alpha(m-1) \dot{\gamma} \dot{\beta}(n-1)} & =0, & \nabla^{\gamma \dot{\gamma}} \Pi^{[m, n]} \phi_{\gamma \alpha(m-1) \dot{\gamma} \dot{\beta}(n-1)} & =0 .
\end{aligned}
$$

Using (4.18a), one can express the higher-spin Weyl tensors as

$$
\begin{aligned}
& \hat{\mathfrak{C}}_{\alpha(m+n)}=\left(\square_{c}\right)^{\frac{n}{2}} \Delta_{\alpha_{1}} \dot{\beta}_{1} \ldots \Delta_{\alpha_{n}} \dot{\beta}_{n} \Pi^{(m, n)} \phi_{\alpha_{n+1} \ldots \alpha_{n+m} \dot{\beta}_{1} \ldots \dot{\beta}_{n}}, \\
& \check{\mathfrak{C}}_{\alpha(m+n)}=\left(\square_{c}\right)^{\frac{m}{2}} \Delta_{\alpha_{1}} \dot{\beta}_{1} \cdots \Delta_{\alpha_{m}}{ }^{\dot{\beta}_{m}} \Pi^{(n, m)} \bar{\phi}_{\alpha_{m+1} \ldots \alpha_{m+n} \dot{\beta}_{1} \ldots \dot{\beta}_{m}} .
\end{aligned}
$$

We note that due to $(4.21 \mathrm{~b})$, both $\hat{\mathfrak{C}}_{\alpha(m+n)}$ and $\check{\mathfrak{C}}_{\alpha(m+n)}$ as written above are totally symmetric.

\footnotetext{
${ }^{9}$ The operator $\Delta_{\alpha \dot{\beta}}$ is a generalisation of the flat-space one used in $[75,76]$ to construct (super)projectors. For two special cases, $m=n$ and $m=n+1$, the projection operators defined by (4.18) are equivalent to the Behrends-Fronsdal projection operators [77, 78].
} 
If we once again assume that $m \geq n$, we may use the descendants (4.9) and the projectors (4.18) to give two new realisations of the CHS action ${ }^{10}$ (4.13),

$$
\begin{aligned}
S_{\mathrm{CHS}}^{(m, n)}[\phi, \bar{\phi}] & =\mathrm{i}^{m+n} \int \mathrm{d}^{4} x e \bar{\phi}^{\alpha(n) \dot{\beta}(m)} \hat{\mathfrak{B}}_{\alpha(n) \dot{\beta}(m)}+\text { c.c. } \\
& =\mathrm{i}^{m+n} \int \mathrm{d}^{4} x e \bar{\phi}^{\alpha(n) \dot{\beta}(m)}\left(\square_{c}\right)^{n} \nabla_{\dot{\beta}_{n+1}} \alpha_{n+1} \ldots \nabla_{\dot{\beta}_{m}}{ }^{\alpha_{m}} \Pi^{(m, n)} \phi_{\alpha(m) \dot{\beta}(n)}+\text { c.c. }
\end{aligned}
$$

When $m=n=s$, the prepotential may be chosen to be real,

$$
h_{\alpha(s) \dot{\alpha}(s)}:=\phi_{\alpha(s) \dot{\alpha}(s)}=\bar{h}_{\alpha(s) \dot{\alpha}(s)} .
$$

In this case there is only one higher-spin Weyl tensor $\hat{\mathfrak{C}}_{\alpha(2 s)}=\check{\mathfrak{C}}_{\alpha(2 s)}=\mathfrak{C}_{\alpha(2 s)}$, and one higher-spin Bach tensor $\hat{\mathfrak{B}}_{\alpha(s) \dot{\beta}(s)}=\check{\mathfrak{B}}_{\alpha(s) \dot{\beta}(s)}=\mathfrak{B}_{\alpha(s) \dot{\beta}(s)}=\overline{\mathfrak{B}}_{\alpha(s) \dot{\beta}(s)}$,

$$
\begin{aligned}
\mathfrak{C}_{\alpha(2 s)} & =\nabla_{\left(\alpha_{1}\right.} \dot{\beta}_{1} \ldots \nabla_{\alpha_{s}}^{\dot{\beta}_{s}} h_{\left.\alpha_{s+1} \ldots \alpha_{2 s}\right)} \dot{\beta}_{1} \ldots \dot{\beta}_{s} \\
\mathfrak{B}_{\alpha(s) \dot{\beta}(s)} & =\nabla_{\left(\dot{\beta}_{1}\right.}{ }^{\gamma_{1}} \ldots \nabla_{\left.\dot{\beta}_{s}\right)} \gamma_{s} \mathfrak{C}_{\alpha_{1} \ldots \alpha_{s} \gamma_{1} \ldots \gamma_{s}},
\end{aligned}
$$

and the action (4.23) assumes the simple form

$$
S_{\mathrm{CHS}}^{(s, s)}[h]=2(-1)^{s} \int \mathrm{d}^{4} x e h^{\alpha(s) \dot{\beta}(s)}\left(\square_{c}\right)^{s} \Pi^{(s, s)} h_{\alpha(s) \dot{\beta}(s)} .
$$

Finally, when $m-1=n=s$ the action (4.23) becomes

$$
S_{\mathrm{CHS}}^{(s+1, s)}=(-1)^{s} \mathrm{i} \int \mathrm{d}^{4} x e \bar{\phi}^{\alpha(s) \dot{\beta}(s+1)} \nabla_{\dot{\beta}_{s+1}}{ }^{\alpha_{s+1}}\left(\square_{c}\right)^{s} \Pi^{(s+1, s)} \phi_{\alpha(s+1) \dot{\beta}(s)}+\text { c.c. }
$$

In the case of Minkowski space, the actions (4.27) and (4.28) coinicde with those proposed by Fradkin and Tseytlin [1].

\subsection{Generalised CHS models}

As a simple extension of the previous constructions, we now consider a generalised gauge field $\phi_{\alpha(m) \dot{\alpha}(n)}^{(l)}$ which is primary and defined modulo gauge transformations of depth $l,{ }^{11}$

$$
\delta_{\lambda} \phi_{\alpha(m) \dot{\alpha}(n)}^{(l)}=\nabla_{\left(\alpha _ { 1 } \left(\dot{\alpha}_{1}\right.\right.} \cdots \nabla_{\alpha_{l} \dot{\alpha}_{l}} \lambda_{\left.\left.\alpha_{l+1} \ldots \alpha_{m}\right) \dot{\alpha}_{l+1} \ldots \dot{\alpha}_{n}\right)},
$$

with $l$ a positive integer, $1 \leq l \leq \min (m, n)$. Using an identity similar to (4.8), one may show that by requiring the gauge parameter $\lambda_{\alpha(m-l) \dot{\alpha}(n-l)}$ to also be primary, the dimension of $\phi_{\alpha(m) \dot{\alpha}(n)}^{(l)}$ is fixed to $(l+1)-\frac{1}{2}(m+n)$. The conformal properties of $\phi_{\alpha(m) \dot{\alpha}(n)}^{(l)}$ may then be summarised by

$$
K_{\beta \dot{\beta}} \phi_{\alpha(m) \dot{\alpha}(n)}^{(l)}=0, \quad \mathbb{D} \phi_{\alpha(m) \dot{\alpha}(n)}^{(l)}=\left((l+1)-\frac{1}{2}(m+n)\right) \phi_{\alpha(m) \dot{\alpha}(n)}^{(l)} .
$$

\footnotetext{
${ }^{10}$ See appendix $\mathrm{D}$ for a discussion on the technical issue of integration by parts.

${ }^{11}$ Such gauge transformations occur in the description of partially massless fields in diverse dimensions [54-69]. Special families of generalised CHS models were studied in [10, 12, 72, 79-81].
} 
As was done earlier in the case $l=1$, from $\phi_{\alpha(m) \dot{\alpha}(n)}^{(l)}$ we may construct generalised higher-spin Weyl tensors $\hat{\mathfrak{C}}_{\alpha(m+n-l+1) \dot{\alpha}(l-1)}^{(l)}(\phi)$ and $\check{\mathfrak{C}}_{\alpha(m+n-l+1) \dot{\alpha}(l-1)}^{(l)}(\bar{\phi})$ possessing the following conformal properties,

$$
\begin{array}{ll}
K_{\beta \dot{\beta}} \hat{\mathfrak{C}}_{\alpha(m+n-l+1) \dot{\alpha}(l-1)}^{(l)}=0, & \mathbb{D} \hat{\mathfrak{C}}_{\alpha(m+n-l+1) \dot{\alpha}(l-1)}^{(l)}=\left(2-\frac{1}{2}(m-n)\right) \hat{\mathfrak{C}}_{\alpha(m+n-l+1) \dot{\alpha}(l-1)}^{(l)}, \\
K_{\beta \dot{\beta}} \check{\mathfrak{C}}_{\alpha(m+n-l+1) \dot{\alpha}(l-1)}^{(l)}=0, & \mathbb{D} \check{\mathfrak{C}}_{\alpha(m+n-l+1) \dot{\alpha}(l-1)}^{(l)}=\left(2-\frac{1}{2}(n-m)\right) \check{\mathfrak{C}}_{\alpha(m+n-l+1) \dot{\alpha}(l-1)}^{(l)} .
\end{array}
$$

In a general curved space one may show, using the identity (4.8), that these properties are satisfied by the following expressions:

$$
\begin{aligned}
& \hat{\mathfrak{C}}_{\alpha(m+n-l+1) \dot{\alpha}(l-1)}^{(l)}=\nabla_{\left(\alpha_{1}\right.} \dot{\beta}_{1} \ldots \nabla_{\alpha_{n-l+1}} \dot{\beta}_{n-l+1} \phi_{\left.\alpha_{n-l+2} \ldots \alpha_{m+n-l+1}\right)}^{(l)} \dot{\beta}_{1} \ldots \dot{\beta}_{n-l+1} \dot{\alpha}_{1} \ldots \dot{\alpha}_{l-1} \\
& \check{\mathfrak{C}}_{\alpha(m+n-l+1) \dot{\alpha}(l-1)}^{(l)}=\nabla_{\left(\alpha_{1}\right.} \dot{\beta}_{1} \ldots \nabla_{\alpha_{m-l+1}} \dot{\beta}_{m-l+1} \bar{\phi}_{\left.\alpha_{m-l+2} \ldots \alpha_{m+n-l+1}\right)}^{(l)} \dot{\beta}_{1} \ldots \dot{\beta}_{m-l+1} \dot{\alpha}_{1} \ldots \dot{\alpha}_{l-1}
\end{aligned}
$$

Associated with these generalised higher-spin Weyl tensors is the action

$$
S_{\mathrm{CHS}}^{(m, n, l)}=\mathrm{i}^{m+n} \int \mathrm{d}^{4} x e \hat{\mathfrak{C}}_{(l)}^{\alpha(m+n-l+1) \dot{\alpha}(l-1)} \check{\mathfrak{C}}_{\alpha(m+n-l+1) \dot{\alpha}(l-1)}^{(l)}+\text { c.c. },
$$

which is invariant under the conformal gauge group $\mathcal{G}$.

In general, if the background Weyl tensor $C_{a b c d}$ is non-vanishing, the primary descendants (4.32) are not invariant under the gauge transformations (4.29). However, in any conformally flat space the generalised higher-spin Weyl tensors (4.32) prove to be gauge invariant

$$
C_{a b c d}=0 \quad \Longrightarrow \quad \delta_{\lambda} \hat{\mathfrak{C}}_{\alpha(m+n-l+1) \dot{\alpha}(l-1)}^{(l)}=\delta_{\lambda} \check{\mathfrak{C}}_{\alpha(m+n-l+1) \dot{\alpha}(l-1)}^{(l)}=0,
$$

and hence so too is the action (4.33),

$$
C_{a b c d}=0 \quad \Longrightarrow \quad \delta_{\lambda} S_{\mathrm{CHS}}^{(m, n, l)}=0 .
$$

The equations of motion that follow from (4.33) are the vanishing of the generalised higher-spin Bach tensors,

$$
\begin{aligned}
& \hat{\mathfrak{B}}_{\alpha(n) \dot{\alpha}(m)}^{(l)}=\nabla_{\left(\dot{\alpha}_{1}\right.} \beta_{1} \ldots \nabla_{\dot{\alpha}_{m-l+1}} \beta_{m-l+1} \hat{\mathfrak{C}}_{\left.\beta_{1} \ldots \beta_{m-l+1} \alpha_{1} \ldots \alpha_{n} \dot{\alpha}_{m-l+2} \ldots \dot{\alpha}_{m}\right)}^{(l)}, \\
& \check{\mathfrak{B}}_{\alpha(m) \dot{\alpha}(n)}^{(l)}=\nabla_{\left(\dot{\alpha}_{1}\right.} \beta_{1} \ldots \nabla_{\dot{\alpha}_{n-l+1}} \beta_{n-l+1} \check{\mathfrak{C}}_{\left.\beta_{1} \ldots \beta_{n-l+1} \alpha_{1} \ldots \alpha_{m} \dot{\alpha}_{n-l+2} \ldots \dot{\alpha}_{n}\right)}^{(l)} .
\end{aligned}
$$

Both (4.36a) and (4.36b) are primary in any curved spacetime. In a conformally flat spacetime, they satisfy the $l$-extended versions of the properties (4.16), namely:

1. $\hat{\mathfrak{B}}_{\alpha(n) \dot{\alpha}(m)}^{(l)}$ and $\check{\mathfrak{B}}_{\alpha(m) \dot{\alpha}(n)}^{(l)}$ are invariant under the gauge transformations (4.29),

$$
\delta_{\lambda} \hat{\mathfrak{B}}_{\alpha(n) \dot{\alpha}(m)}^{(l)}=\delta_{\lambda} \check{\mathfrak{B}}_{\alpha(m) \dot{\alpha}(n)}^{(l)}=0 .
$$


2. $\hat{\mathfrak{B}}_{\alpha(n) \dot{\alpha}(m)}^{(l)}$ and $\check{\mathfrak{B}}_{\alpha(m) \dot{\alpha}(n)}^{(l)}$ are partially conserved,

$$
\nabla^{\beta_{1} \dot{\beta}_{1}} \ldots \nabla^{\beta_{l} \dot{\beta}_{l} \hat{\mathfrak{B}}_{\beta(l) \alpha(n-l) \dot{\beta}(l) \dot{\alpha}(m-l)}^{(l)}}=\nabla^{\beta_{1} \dot{\beta}_{1}} \ldots \nabla^{\beta_{l} \dot{\beta}_{l} \check{\mathfrak{B}}_{\beta(l) \alpha(m-l) \dot{\beta}(l) \dot{\alpha}(n-l)}^{(l)}}=0 .
$$

3. The complex conjugates of $\hat{\mathfrak{B}}_{\alpha(n) \dot{\beta}(m)}^{(l)}$ and $\check{\mathfrak{B}}_{\alpha(m) \dot{\beta}(n)}^{(l)}$ satisfy

$$
\overline{\hat{\mathfrak{B}}}_{\alpha(m) \dot{\alpha}(n)}^{(l)}=\check{\mathfrak{B}}_{\alpha(m) \dot{\alpha}(n)}^{(l)}, \quad \check{\mathfrak{\mathfrak { B }}}_{\alpha(n) \dot{\alpha}(m)}^{(l)}=\hat{\mathfrak{B}}_{\alpha(n) \dot{\alpha}(m)}^{(l)} .
$$

As was done in the 3D case, we can once again ask which values of $l$ yield second-order Lagrangians in the action (4.33). ${ }^{12}$ A similar analysis reveals that second order models exist only for bosonic spin. These models are of maximal depth and have $l=m=n=s$ with $s=2,3, \ldots$. They were first described in $[72,79]$.

It is of interest to provide a more detailed analysis of the maximal depth spin-2 model in a general curved space. Setting $l=m=n=2$ and denoting $\mathfrak{C}_{\alpha(3) \dot{\alpha}}:=\hat{\mathfrak{C}}_{\alpha(3) \dot{\alpha}}^{(2)}=\check{\mathfrak{C}}_{\alpha(3) \dot{\alpha}}^{(2)}$ and $h_{\alpha(2) \dot{\alpha}(2)}:=\phi_{\alpha(2) \dot{\alpha}(2)}^{(2)}=\bar{h}_{\alpha(2) \dot{\alpha}(2)}$, the action (4.33) takes the form

$$
S_{\mathrm{CHS}}^{(2,2,2)}=\int \mathrm{d}^{4} x e \mathfrak{C}^{\alpha(3) \dot{\alpha}} \mathfrak{C}_{\alpha(3) \dot{\alpha}}+\text { c.c. }, \quad \mathfrak{C}_{\alpha(3) \dot{\alpha}}=\nabla_{\left(\alpha_{1}\right.}{ }^{\dot{\beta}} h_{\left.\alpha_{2} \alpha_{3}\right) \dot{\alpha} \dot{\beta}} .
$$

We can still add a non-minimal term to this action whilst respecting its $\mathcal{G}$-invariance,

$$
\begin{gathered}
\widetilde{S}_{\mathrm{CHS}}^{(2,2,2)}=S_{\mathrm{CHS}}^{(2,2,2)}+\omega S_{\mathrm{NM}}^{(2,2,2)} \\
S_{\mathrm{NM}}^{(2,2,2)}=\int \mathrm{d}^{4} x e C^{\alpha(2) \beta(2)} h_{\alpha(2)}{ }^{\dot{\alpha}(2)} h_{\beta(2) \dot{\alpha}(2)}+\text { c.c. },
\end{gathered}
$$

where $\omega$ is some constant and $C^{\alpha(2) \beta(2)}$ is the self-dual part of the Weyl tensor, eq. (4.3). We find that under the gauge transformations

$$
\delta_{\lambda} h_{\alpha(2) \dot{\alpha}(2)}=\nabla_{\left(\alpha _ { 1 } \left(\dot{\alpha}_{1}\right.\right.} \nabla_{\left.\left.\alpha_{2}\right) \dot{\alpha}_{2}\right)} \lambda,
$$

the deformed action (4.39a) varies as

$$
\begin{aligned}
\delta_{\lambda} \widetilde{S}_{\mathrm{CHS}}^{(2,2) 2)}=-2 \int \mathrm{d}^{4} x e \lambda\{ & (1+\omega) C_{\alpha(3) \delta} \nabla_{\dot{\alpha}}{ }^{\delta} \mathfrak{C}^{\alpha(3) \dot{\alpha}}+2(1+\omega) \mathfrak{C}^{\alpha(3) \dot{\alpha}} \nabla_{\dot{\alpha}}{ }^{\delta} C_{\alpha(3) \delta} \\
& \left.-\omega B^{\alpha(2) \dot{\alpha}(2)} h_{\alpha(2) \dot{\alpha}(2)}\right\}+ \text { c.c. },
\end{aligned}
$$

where $B_{\alpha(2) \dot{\alpha}(2)}$ is the Bach tensor,

$$
B_{\alpha(2) \dot{\alpha}(2)}=\nabla_{\left(\dot{\alpha}_{1}\right.}^{\beta_{1}} \nabla_{\left.\dot{\alpha}_{2}\right)}^{\beta_{2}} C_{\alpha(2) \beta(2)}=\nabla_{\left(\alpha_{1}\right.} \dot{\beta}_{1} \nabla_{\left.\alpha_{2}\right)} \dot{\beta}_{2} \bar{C}_{\dot{\alpha}(2) \dot{\beta}(2)}=\bar{B}_{\alpha(2) \dot{\alpha}(2)} .
$$

Therefore, if we choose $\omega=-1$ then it follows that (4.39a) is gauge invariant in any Bach-flat spacetime,

$$
B_{\alpha(2) \dot{\alpha}(2)}=\left.0 \quad \Longrightarrow \quad \delta_{\lambda} \widetilde{S}_{\mathrm{CHS}}^{(2,2,2)}\right|_{\omega=-1}=0 .
$$

\footnotetext{
${ }^{12}$ First-order models in this context are not well defined.
} 
This model was discussed in ref. [12], where the authors concluded that gauge invariance could be extended to any Einstein space. Although this statement is true, as we have just shown, it may be extended even further to Bach-flat backgrounds. Perhaps the reason they did not arrive at this conclusion was because they demanded gauge transformations of the type

$$
\delta_{\lambda} h_{a b}=\mathcal{D}_{a} \mathcal{D}_{b} \lambda-\frac{1}{4} \eta_{a b} \square \lambda .
$$

However, the right-hand side does not preserve its form under Weyl transformations. The correct gauge transformation in a general curved background is the degauged version of (4.40), which in vector notation reads

$$
\delta_{\lambda} h_{a b}=\left(\mathcal{D}_{a} \mathcal{D}_{b}-\frac{1}{2} R_{a b}\right) \lambda-\frac{1}{4} \eta_{a b}\left(\square-\frac{1}{2} R\right) \lambda .
$$

The two expressions coincide in the case of Einstein spaces.

\subsection{Degauging}

To conclude this section, we discuss the $4 D$ degauging procedure, which turns out to be much more tractable than the $3 D$ one. In the gauge $(2.25)$, the conformal covariant derivative reads

$$
\nabla_{\alpha \dot{\alpha}}=\mathcal{D}_{\alpha \dot{\alpha}}-\frac{1}{4} P_{\alpha \dot{\alpha}},{ }^{\beta \dot{\beta}} K_{\beta \dot{\beta}}
$$

The Schouten tensor may be decomposed into irreducible components as

$$
P_{\alpha \dot{\alpha}, \beta \dot{\beta}}=\frac{1}{2} R_{\alpha \beta \dot{\alpha} \dot{\beta}}-\frac{1}{12} \varepsilon_{\alpha \beta} \varepsilon_{\dot{\alpha} \dot{\beta}} R
$$

where $R_{\alpha \dot{\alpha} \dot{\alpha}}=\left(\sigma^{a}\right)_{\alpha \dot{\alpha}}\left(\sigma^{b}\right)_{\beta \dot{\beta}}\left(R_{a b}-\frac{1}{4} \eta_{a b} R\right)=R_{(\alpha \beta)(\dot{\alpha} \dot{\beta})}$ is the traceless Ricci tensor. The aim is then to express all descendants in terms of the torsion-free Lorentz covariant derivative, the curvature and the prepotential.

Using the identity (4.8) and the degauged covariant derivative, it is possible to show that the following identity holds true

$$
\begin{aligned}
\mathcal{D}_{\left(\alpha_{1}\right.} \dot{\beta}_{1} \ldots \mathcal{D}_{\alpha_{j-1}} \dot{\beta}_{j-1} \nabla_{\alpha_{j}} \dot{\beta}_{j} \ldots \nabla_{\alpha_{n}} \dot{\beta}_{n} \phi_{\left.\alpha_{n+1} \ldots \alpha_{n+m}\right) \dot{\beta}_{1} \ldots \dot{\beta}_{n}} \\
=\mathcal{D}_{\left(\alpha_{1}\right.} \dot{\beta}_{1} \ldots \mathcal{D}_{\alpha_{j-1}} \dot{\beta}_{j-1}\left\{\mathcal{D}_{\alpha_{j}} \dot{\beta}_{j} \nabla_{\alpha_{j+1}} \dot{\beta}_{j+1} \ldots \nabla_{\alpha_{n}} \dot{\beta}_{n}\right. \\
\left.\quad-\frac{1}{2} j(n-j) R_{\alpha_{j} \alpha_{j+1}} \dot{\beta}_{j} \dot{\beta}_{j+1} \nabla_{\alpha_{j+2}} \dot{\beta}_{j+2} \ldots \nabla_{\alpha_{n}} \dot{\beta}_{n}\right\} \phi_{\left.\alpha_{n+1} \ldots \alpha_{n+m}\right) \dot{\beta}_{1} \ldots \dot{\beta}_{n}} .
\end{aligned}
$$

Therefore, in any background spacetime with a vanishing traceless Ricci tensor, or in other words an Einstein space,

$$
R_{\alpha \beta \dot{\alpha} \dot{\beta}}=0 \quad \Longleftrightarrow \quad R_{a b}=\lambda \eta_{a b},
$$

the degauging procedure is trivial and we obtain ${ }^{13}$

$$
\begin{aligned}
\hat{\mathfrak{C}}_{\alpha(m+n)} & =\mathcal{D}_{\left(\alpha_{1}\right.}{ }^{\dot{\beta}_{1}} \ldots \mathcal{D}_{\alpha_{n}}{ }^{\dot{\beta}_{n}} \phi_{\left.\alpha_{n+1} \ldots \alpha_{n+m}\right) \dot{\beta}_{1} \ldots \dot{\beta}_{n}}, \\
\check{\mathfrak{C}}_{\alpha(m+n)} & =\mathcal{D}_{\left(\alpha_{1}\right.}{ }^{\dot{\beta}_{1}} \ldots \mathcal{D}_{\alpha_{m}}{ }^{\dot{\beta}_{m}} \bar{\phi}_{\left.\alpha_{m+1} \ldots \alpha_{m+n}\right)} \dot{\beta}_{1} \ldots \dot{\beta}_{m}
\end{aligned}
$$

\footnotetext{
${ }^{13}$ An identity similar to (4.48) holds with $\phi_{\alpha(m) \dot{\alpha}(n)}$ replaced with $\hat{\mathfrak{C}}_{\alpha(m+n)}$.
} 


$$
\begin{aligned}
\hat{\mathfrak{B}}_{\alpha(n) \dot{\beta}(m)} & =\mathcal{D}_{\left(\dot{\beta}_{1}\right.}{ }^{\gamma_{1}} \cdots \mathcal{D}_{\left.\dot{\beta}_{m}\right)}{ }^{\gamma_{m}} \hat{\mathfrak{C}}_{\alpha_{1} \ldots \alpha_{n} \gamma_{1} \ldots \gamma_{m}}, \\
\check{\mathfrak{B}}_{\alpha(m) \dot{\beta}(n)} & =\mathcal{D}_{\left(\dot{\beta}_{1}\right.} \gamma_{1} \cdots \mathcal{D}_{\left.\dot{\beta}_{n}\right)}{ }^{\gamma_{n}} \check{\mathfrak{C}}_{\alpha_{1} \ldots \alpha_{m} \gamma_{1} \ldots \gamma_{n}} .
\end{aligned}
$$

In the case where $R_{\alpha(2) \dot{\alpha}(2)}$ does not vanish we have not yet been able to obtain a closed-form expression for the degauged version of any of the above tensors, for arbitrary $m$ and $n$. However, the expressions for $\mathfrak{C}_{\alpha(2 s)}$ with $s=2,3,4,5$ are as follows:

$$
\begin{aligned}
& \mathfrak{C}_{\alpha(4)}=\mathcal{D}_{\left(\alpha_{1}\right.}{ }^{\dot{\beta}_{1}} \mathcal{D}_{\alpha_{2}}{ }^{\dot{\beta}_{2}} h_{\left.\alpha_{3} \alpha_{4}\right) \dot{\beta}_{1} \dot{\beta}_{2}}-\frac{1}{2} R_{\left(\alpha_{1} \alpha_{2}\right.} \dot{\beta}_{1} \dot{\beta}_{2} h_{\left.\alpha_{3} \alpha_{4}\right) \dot{\beta}_{1} \dot{\beta}_{2}}, \\
& \mathfrak{C}_{\alpha(6)}=\mathcal{D}_{\left(\alpha_{1}\right.}{ }^{\dot{\beta}_{1}} \mathcal{D}_{\alpha_{2}}{ }^{\dot{\beta}_{2}} \mathcal{D}_{\alpha_{3}}{ }^{\dot{\beta}_{3}} h_{\left.\alpha_{4} \alpha_{5} \alpha_{6}\right) \dot{\beta}_{1} \dot{\beta}_{2} \dot{\beta}_{3}} \\
& \text { - }\left(\mathcal{D}_{\left(\alpha_{1}\right.} \dot{\beta}_{1} R_{\alpha_{2} \alpha_{3}} \dot{\beta}_{2} \dot{\beta}_{3}\right) h_{\left.\alpha_{4} \alpha_{5} \alpha_{6}\right) \dot{\beta}_{1} \dot{\beta}_{2} \dot{\beta}_{3}} \\
& -2 R_{\left(\alpha_{1} \alpha_{2}\right.}^{\dot{\beta}_{1} \dot{\beta}_{2}} \mathcal{D}_{\alpha_{3}}{ }^{\dot{\beta}_{3}} h_{\left.\alpha_{4} \alpha_{5} \alpha_{6}\right) \dot{\beta}_{1} \dot{\beta}_{2} \dot{\beta}_{3}}, \\
& \mathfrak{C}_{\alpha(8)}=\mathcal{D}_{\left(\alpha_{1}\right.}{ }^{\dot{\beta}_{1}} \mathcal{D}_{\alpha_{2}}{ }^{\dot{\beta}_{2}} \mathcal{D}_{\alpha_{3}}{ }^{\dot{\beta}_{3}} \mathcal{D}_{\alpha_{4}}{ }^{\dot{\beta}_{4}} h_{\left.\alpha_{5} \alpha_{6} \alpha_{7} \alpha_{8}\right) \dot{\beta}_{1} \dot{\beta}_{2} \dot{\beta}_{3} \dot{\beta}_{4}} \\
& -\frac{3}{2}\left(\mathcal{D}_{\left(\alpha_{1}\right.}{ }^{\dot{\beta}_{1}} \mathcal{D}_{\alpha_{2}}{ }^{\dot{\beta}_{2}} R_{\alpha_{3} \alpha_{4}}{ }^{\dot{\beta}_{3} \dot{\beta}_{4}}\right) h_{\left.\alpha_{5} \alpha_{6} \alpha_{7} \alpha_{8}\right) \dot{\beta}_{1} \dot{\beta}_{2} \dot{\beta}_{3} \dot{\beta}_{4}} \\
& -5\left(\mathcal{D}_{\left(\alpha_{1}\right.}{ }^{\dot{\beta}_{1}} R_{\alpha_{2} \alpha_{3}} \dot{\beta}_{2} \dot{\beta}_{3}\right) \mathcal{D}_{\alpha_{4}}{ }^{\dot{\beta}_{4}} h_{\left.\alpha_{5} \alpha_{6} \alpha_{7} \alpha_{8}\right) \dot{\beta}_{1} \dot{\beta}_{2} \dot{\beta}_{3} \dot{\beta}_{4}} \\
& -5 R_{\left(\alpha_{1} \alpha_{2}\right.}{ }_{1}^{\dot{\beta}_{1} \dot{\beta}_{2}} \mathcal{D}_{\alpha_{3}}{ }^{\dot{\beta}_{3}} \mathcal{D}_{\alpha_{4}}{ }^{\dot{\beta}_{4}} h_{\left.\alpha_{5} \alpha_{6} \alpha_{7} \alpha_{8}\right) \dot{\beta}_{1} \dot{\beta}_{2} \dot{\beta}_{3} \dot{\beta}_{4}} \\
& +\frac{9}{4} R_{\left(\alpha_{1} \alpha_{2}\right.}{ }^{\dot{\beta}_{1} \dot{\beta}_{2}} R_{\alpha_{3} \alpha_{4}}{ }^{\dot{\beta}_{3} \dot{\beta}_{4}} h_{\left.\alpha_{5} \alpha_{6} \alpha_{7} \alpha_{8}\right) \dot{\beta}_{1} \dot{\beta}_{2} \dot{\beta}_{3} \dot{\beta}_{4}}, \\
& \mathfrak{C}_{\alpha(10)}=\mathcal{D}_{\left(\alpha_{1}\right.}{ }^{\dot{\beta}_{1}} \ldots \mathcal{D}_{\alpha_{5}}{ }^{\dot{\beta}_{5}} h_{\left.\alpha_{6} \ldots \alpha_{10}\right) \dot{\beta}_{1} \ldots \dot{\beta}_{5}} \\
& -2\left(\mathcal{D}_{\left(\alpha_{1}\right.}{ }^{\dot{\beta}_{1}} \mathcal{D}_{\alpha_{2}}{ }^{\dot{\beta}_{2}} \mathcal{D}_{\alpha_{3}}{ }^{\dot{\beta}_{3}} R_{\alpha_{4} \alpha_{5}} \dot{\beta}_{4} \dot{\beta}_{5}\right) h_{\left.\alpha_{6} \ldots \alpha_{10}\right) \dot{\beta}_{1} \ldots \dot{\beta}_{5}} \\
& -9\left(\mathcal{D}_{\left(\alpha_{1}\right.}{ }^{\dot{\beta}_{1}} \mathcal{D}_{\alpha_{2}}{ }^{\dot{\beta}_{2}} R_{\alpha_{3} \alpha_{4}}{ }^{\dot{\beta}_{3} \dot{\beta}_{4}}\right) \mathcal{D}_{\alpha_{5}}{ }^{\dot{\beta}_{5}} h_{\left.\alpha_{6} \ldots \alpha_{10}\right)} \dot{\beta}_{1} \ldots \dot{\beta}_{5} \\
& -15\left(\mathcal{D}_{\left(\alpha_{1}\right.}{ }^{\dot{\beta}_{1}} R_{\alpha_{2} \alpha_{3}}{ }^{\dot{\beta}_{2} \dot{\beta}_{3}}\right) \mathcal{D}_{\alpha_{4}}{ }^{\dot{\beta}_{4}} \mathcal{D}_{\alpha_{5}}{ }^{\dot{\beta}_{5}} h_{\left.\alpha_{6} \ldots \alpha_{10}\right) \dot{\beta}_{1} \ldots \dot{\beta}_{5}} \\
& \text { - } 10 R_{\left(\alpha_{1} \alpha_{2}\right.}{ }^{\dot{\beta}_{1} \dot{\beta}_{2}} \mathcal{D}_{\alpha_{3}}{ }^{\dot{\beta}_{3}} \mathcal{D}_{\alpha_{4}}{ }^{\dot{\beta}_{4}} \mathcal{D}_{\alpha_{5}}{ }^{\dot{\beta}_{5}} h_{\left.\alpha_{6} \ldots \alpha_{10}\right) \dot{\beta}_{1} \ldots \dot{\beta}_{5}} \\
& +16 R_{\left(\alpha_{1} \alpha_{2}\right.}{ }^{\dot{\beta}_{1} \dot{\beta}_{2}}\left(\mathcal{D}_{\alpha_{3}}{ }^{\dot{\beta}_{3}} R_{\alpha_{4} \alpha_{5}}{ }^{\dot{\beta}_{4} \dot{\beta}_{5}}\right) h_{\left.\alpha_{6} \ldots \alpha_{10}\right) \dot{\beta}_{1} \ldots \dot{\beta}_{5}} \\
& +16 R_{\left(\alpha_{1} \alpha_{2}\right.}{ }^{\dot{\beta}_{1} \dot{\beta}_{2}} R_{\alpha_{3} \alpha_{4}}{ }^{\dot{\beta}_{3} \dot{\beta}_{4}} \mathcal{D}_{\alpha_{5}} \dot{\beta}_{5} h_{\left.\alpha_{6} \ldots \alpha_{10}\right) \dot{\beta}_{1} \ldots \dot{\beta}_{5}} .
\end{aligned}
$$

Modulo terms involving the background Weyl tensor, eq. (4.51a) proves to coincide with the linearised self-dual Weyl tensor $C_{\alpha(4)}$.

The expressions for $\hat{\mathfrak{C}}_{\alpha(m+n)}$ with $m-1=n=s$ for $s=1,2,3,4$ are as follows:

$$
\begin{aligned}
& \hat{\mathfrak{C}}_{\alpha(3)}=\mathcal{D}_{\left(\alpha_{1}\right.} \dot{\beta}_{1} \phi_{\left.\alpha_{2} \alpha_{3}\right) \dot{\beta}_{1}}, \\
& \hat{\mathfrak{C}}_{\alpha(5)}=\mathcal{D}_{\left(\alpha_{1}\right.} \dot{\beta}_{1} \mathcal{D}_{\alpha_{2}} \dot{\beta}_{2} \phi_{\left.\alpha_{3} \alpha_{4} \alpha_{5}\right) \dot{\beta}_{1} \dot{\beta}_{2}}-\frac{1}{2} R_{\left(\alpha_{1} \alpha_{2}\right.} \dot{\beta}_{1} \dot{\beta}_{2} \phi_{\left.\alpha_{3} \alpha_{4} \alpha_{5}\right) \dot{\beta}_{1} \dot{\beta}_{2}}, \\
& \hat{\mathfrak{C}}_{\alpha(7)}=\mathcal{D}_{\left(\alpha_{1}\right.}{ }^{\dot{\beta}_{1}} \mathcal{D}_{\alpha_{2}}{ }^{\dot{\beta}_{2}} \mathcal{D}_{\alpha_{3}}{ }^{\dot{\beta}_{3}} \phi_{\left.\alpha_{4} \alpha_{5} \alpha_{6} \alpha_{7}\right) \dot{\beta}_{1} \dot{\beta}_{2} \dot{\beta}_{3}} \\
& \text { - }\left(\mathcal{D}_{\left(\alpha_{1}\right.}{ }^{\dot{\beta}_{1}} R_{\alpha_{2} \alpha_{3}} \dot{\beta}_{2} \dot{\beta}_{3}\right) \phi_{\left.\alpha_{4} \alpha_{5} \alpha_{6} \alpha_{7}\right) \dot{\beta}_{1} \dot{\beta}_{2} \dot{\beta}_{3}} \\
& -2 R_{\left(\alpha_{1} \alpha_{2}\right.}{ }^{\dot{\beta}_{1} \dot{\beta}_{2}} \mathcal{D}_{\alpha_{3}}{ }^{\dot{\beta}_{3}} \phi_{\left.\alpha_{4} \alpha_{5} \alpha_{6} \alpha_{7}\right) \dot{\beta}_{1} \dot{\beta}_{2} \dot{\beta}_{3}},
\end{aligned}
$$




$$
\begin{aligned}
\hat{\mathfrak{C}}_{\alpha(9)}= & \mathcal{D}_{\left(\alpha_{1}\right.}{ }^{\dot{\beta}_{1}} \mathcal{D}_{\alpha_{2}}{ }^{\dot{\beta}_{2}} \mathcal{D}_{\alpha_{3}}{ }^{\dot{\beta}_{3}} \mathcal{D}_{\alpha_{4}}{ }^{\dot{\beta}_{4}} \phi_{\left.\alpha_{5} \ldots \alpha_{9}\right) \dot{\beta}_{1} \ldots \dot{\beta}_{4}} \\
& -\frac{3}{2}\left(\mathcal{D}_{\left(\alpha_{1}\right.}{ }^{\dot{\beta}_{1}} \mathcal{D}_{\alpha_{2}}{ }^{\dot{\beta}_{2}} R_{\alpha_{3} \alpha_{4}} \dot{\beta}_{3} \dot{\beta}_{4}\right) \phi_{\left.\alpha_{5} \ldots \alpha_{9}\right) \dot{\beta}_{1} \ldots \dot{\beta}_{4}} \\
& -5\left(\mathcal{D}_{\left(\alpha_{1}\right.}{ }^{\dot{\beta}_{1}} R_{\alpha_{2} \alpha_{3}} \dot{\beta}_{2} \dot{\beta}_{3}\right) \mathcal{D}_{\alpha_{4}}{ }^{\dot{\beta}_{4}} \phi_{\left.\alpha_{5} \ldots \alpha_{9}\right) \dot{\beta}_{1} \ldots \dot{\beta}_{4}} \\
& -5 R_{\left(\alpha_{1} \alpha_{2}\right.}{ }^{\dot{\beta}_{1} \dot{\beta}_{2}} \mathcal{D}_{\alpha_{3}} \dot{\beta}_{3} \mathcal{D}_{\alpha_{4}}{ }^{\dot{\beta}_{4}} \phi_{\left.\alpha_{5} \ldots \alpha_{9}\right) \dot{\beta}_{1} \ldots \dot{\beta}_{4}} \\
& +\frac{9}{4} R_{\left(\alpha_{1} \alpha_{2}\right.}{ }^{\dot{\beta}_{1} \dot{\beta}_{2}} R_{\alpha_{3} \alpha_{4}}{ }^{\dot{\beta}_{3} \dot{\beta}_{4}} \phi_{\left.\alpha_{5} \ldots \alpha_{9}\right) \dot{\beta}_{1} \ldots \dot{\beta}_{4}}
\end{aligned}
$$

Finally, the expressions for $\check{\mathfrak{C}}_{\alpha(m+n)}$ with $m-1=n=s$ for $s=1,2,3,4$ are as follows:

$$
\begin{aligned}
& \check{\mathfrak{C}}_{\alpha(3)}=\mathcal{D}_{\left(\alpha_{1}\right.}{ }^{\dot{\beta}_{1}} \mathcal{D}_{\alpha_{2}} \dot{\beta}_{2} \bar{\phi}_{\left.\alpha_{1}\right) \dot{\beta}_{1} \dot{\beta}_{2}}-\frac{1}{2} R_{\left(\alpha_{1} \alpha_{2}\right.} \dot{\beta}_{1} \dot{\beta}_{2} \bar{\phi}_{\left.\alpha_{3}\right) \dot{\beta}_{1} \dot{\beta}_{2}}, \\
& \check{\mathfrak{C}}_{\alpha(5)}=\mathcal{D}_{\left(\alpha_{1}\right.}{ }^{\dot{\beta}_{1}} \mathcal{D}_{\alpha_{2}}{ }^{\dot{\beta}_{2}} \mathcal{D}_{\alpha_{3}} \dot{\beta}_{3} \bar{\phi}_{\left.\alpha_{4} \alpha_{5}\right) \dot{\beta}_{1} \dot{\beta}_{2} \dot{\beta}_{3}} \\
& -\left(\mathcal{D}_{\left(\alpha_{1}\right.}{ }^{\dot{\beta}_{1}} R_{\alpha_{2} \alpha_{3}}{ }^{\dot{\beta}_{2} \dot{\beta}_{3}}\right) \bar{\phi}_{\left.\alpha_{4} \alpha_{5}\right) \dot{\beta}_{1} \dot{\beta}_{2} \dot{\beta}_{3}} \\
& -2 R_{\left(\alpha_{1} \alpha_{2}\right.}{ }^{\dot{\beta}_{1} \dot{\beta}_{2}} \mathcal{D}_{\alpha_{3}} \dot{\beta}_{3} \bar{\phi}_{\left.\alpha_{4} \alpha_{5}\right) \dot{\beta}_{1} \dot{\beta}_{2} \dot{\beta}_{3}}, \\
& \check{\mathfrak{C}}_{\alpha(7)}=\mathcal{D}_{\left(\alpha_{1}\right.}{ }^{\dot{\beta}_{1}} \mathcal{D}_{\alpha_{2}}{ }^{\dot{\beta}_{2}} \mathcal{D}_{\alpha_{3}}{ }^{\dot{\beta}_{3}} \mathcal{D}_{\alpha_{4}}{ }^{\dot{\beta}_{4}} \bar{\phi}_{\left.\alpha_{5} \alpha_{6} \alpha_{7}\right) \dot{\beta}_{1} \dot{\beta}_{2} \dot{\beta}_{3} \dot{\beta}_{4}} \\
& -\frac{3}{2}\left(\mathcal{D}_{\left(\alpha_{1}\right.}{ }^{\dot{\beta}_{1}} \mathcal{D}_{\alpha_{2}}{ }^{\dot{\beta}_{2}} R_{\alpha_{3} \alpha_{4}}{ }^{\dot{\beta}_{3} \dot{\beta}_{4}}\right) \bar{\phi}_{\left.\alpha_{5} \alpha_{6} \alpha_{7}\right) \dot{\beta}_{1} \dot{\beta}_{2} \dot{\beta}_{3} \dot{\beta}_{4}} \\
& -5\left(\mathcal{D}_{\left(\alpha_{1}\right.} \dot{\beta}_{1} R_{\alpha_{2} \alpha_{3}} \dot{\beta}_{2} \dot{\beta}_{3}\right) \mathcal{D}_{\alpha_{4}} \dot{\beta}_{4} \bar{\phi}_{\left.\alpha_{5} \alpha_{6} \alpha_{7}\right)} \dot{\beta}_{1} \dot{\beta}_{2} \dot{\beta}_{3} \dot{\beta}_{4} \\
& -5 R_{\left(\alpha_{1} \alpha_{2}\right.}{ }^{\dot{\beta}_{1} \dot{\beta}_{2}} \mathcal{D}_{\alpha_{3}}{ }^{\dot{\beta}_{3}} \mathcal{D}_{\alpha_{4}} \dot{\beta}_{4} \bar{\phi}_{\left.\alpha_{5} \alpha_{6} \alpha_{7}\right) \dot{\beta}_{1} \dot{\beta}_{2} \dot{\beta}_{3} \dot{\beta}_{4}} \\
& +\frac{9}{4} R_{\left(\alpha_{1} \alpha_{2}\right.}{ }^{\dot{\beta}_{1} \dot{\beta}_{2}} R_{\alpha_{3} \alpha_{4}} \dot{\beta}_{3} \dot{\beta}_{4} \bar{\phi}_{\left.\alpha_{5} \alpha_{6} \alpha_{7}\right) \dot{\beta}_{1} \dot{\beta}_{2} \dot{\beta}_{3} \dot{\beta}_{4}} \\
& \check{\mathfrak{C}}_{\alpha(9)}=\mathcal{D}_{\left(\alpha_{1}\right.} \dot{\beta}_{1} \ldots \mathcal{D}_{\alpha_{5}} \dot{\beta}_{5} \bar{\phi}_{\left.\alpha_{6} \ldots \alpha_{9}\right)} \dot{\beta}_{1} \ldots \dot{\beta}_{5} \\
& -2\left(\mathcal{D}_{\left(\alpha_{1}\right.}{ }^{\dot{\beta}_{1}} \mathcal{D}_{\alpha_{2}}{ }^{\dot{\beta}_{2}} \mathcal{D}_{\alpha_{3}}{ }^{\dot{\beta}_{3}} R_{\alpha_{4} \alpha_{5}} \dot{\beta}_{4} \dot{\beta}_{5}\right) \bar{\phi}_{\left.\alpha_{6} \ldots \alpha_{9}\right) \dot{\beta}_{1} \ldots \dot{\beta}_{5}} \\
& -9\left(\mathcal{D}_{\left(\alpha_{1}\right.}{ }^{\dot{\beta}_{1}} \mathcal{D}_{\alpha_{2}}{ }^{\dot{\beta}_{2}} R_{\alpha_{3} \alpha_{4}}{ }^{\dot{\beta}_{3} \dot{\beta}_{4}}\right) \mathcal{D}_{\alpha_{5}}{ }^{\dot{\beta}_{5}} \bar{\phi}_{\left.\alpha_{6} \ldots \alpha_{9}\right) \dot{\beta}_{1} \ldots \dot{\beta}_{5}} \\
& -15\left(\mathcal{D}_{\left(\alpha_{1}\right.} \dot{\beta}_{1} R_{\alpha_{2} \alpha_{3}} \dot{\beta}_{2} \dot{\beta}_{3}\right) \mathcal{D}_{\alpha_{4}}{ }^{\dot{\beta}_{4}} \mathcal{D}_{\alpha_{5}}{ }^{\dot{\beta}_{5}} \bar{\phi}_{\left.\alpha_{6} \ldots \alpha_{9}\right) \dot{\beta}_{1} \ldots \dot{\beta}_{5}} \\
& -10 R_{\left(\alpha_{1} \alpha_{2}\right.}{ }^{\dot{\beta}_{1} \dot{\beta}_{2}} \mathcal{D}_{\alpha_{3}}{ }^{\dot{\beta}_{3}} \mathcal{D}_{\alpha_{4}}{ }^{\dot{\beta}_{4}} \mathcal{D}_{\alpha_{5}} \dot{\beta}_{5} \bar{\phi}_{\left.\alpha_{6} \ldots \alpha_{9}\right) \dot{\beta}_{1} \ldots \dot{\beta}_{5}} \\
& +16 R_{\left(\alpha_{1} \alpha_{2}\right.} \dot{\beta}_{1} \dot{\beta}_{2}\left(\mathcal{D}_{\alpha_{3}}{ }^{\dot{\beta}_{3}} R_{\alpha_{4} \alpha_{5}} \dot{\beta}_{4} \dot{\beta}_{5}\right) \bar{\phi}_{\left.\alpha_{6} \ldots \alpha_{9}\right) \dot{\beta}_{1} \ldots \dot{\beta}_{5}} \\
& +16 R_{\left(\alpha_{1} \alpha_{2}\right.}{ }^{\dot{\beta}_{1} \dot{\beta}_{2}} R_{\alpha_{3} \alpha_{4}}{ }^{\dot{\beta}_{3} \dot{\beta}_{4}} \mathcal{D}_{\alpha_{5}}{ }^{\dot{\beta}_{5}} \bar{\phi}_{\left.\alpha_{6} \ldots \alpha_{9}\right) \dot{\beta}_{1} \ldots \dot{\beta}_{5}} .
\end{aligned}
$$

Here we do not attempt to degauge the generalised higher-spin Weyl and Bach tensors introduced in the previous subsection. However, we do note that they will also degauge trivially in any Einstein space.

\section{SCHS theories in three dimensions}

In three dimensions, $\mathcal{N}$-extended conformal supergravity was formulated in superspace as the gauge theory of the superconformal group in [38]. Upon degauging, this formulation reduces to the conventional one, sketched in [82] and fully developed in [83], with the 
local structure group $\mathrm{SL}(2, \mathbb{R}) \times \mathrm{SO}(\mathcal{N})$. The former formulation is known as $\mathcal{N}$-extended conformal superspace, while the latter is often referred to as $\mathrm{SO}(\mathcal{N})$ superspace. In this section we only make use of the conformal superspace formulations for $\mathcal{N}=1,2$ and 3 . To start with, we recall the main facts about the $3 D \mathcal{N}$-extended superconformal algebra and primary superfields in conformal superspace following [38].

The $3 D \mathcal{N}$-extended superconformal algebra, $\mathfrak{o s p}(\mathcal{N} \mid 4, \mathbb{R})$, contains bosonic and fermionic generators. Its even part $\mathfrak{s o}(3,2) \oplus \mathfrak{s o}(\mathcal{N})$ includes the generators of $\mathfrak{s o}(\mathcal{N})$, $N_{K L}=-N_{L K}$, where $K, L=1, \ldots, \mathcal{N}$, in addition to the generators of the conformal group described in section 2.1. Their commutation relations are:

$$
\left[N_{K L}, N^{I J}\right]=2 \delta_{[K}^{I} N_{L]}^{J}-2 \delta_{[K}^{J} N_{L]}^{I} .
$$

The odd part of $\mathfrak{o s p}(\mathcal{N} \mid 4, \mathbb{R})$ is spanned by the $Q$-supersymmetry $\left(Q_{\alpha}^{I}\right)$ and $S$-supersymmetry $\left(S_{\alpha}^{I}\right)$ generators. In accordance with [38], the fermionic operators $Q_{\alpha}^{I}$ obey the algebra

$$
\begin{aligned}
& \left\{Q_{\alpha}^{I}, Q_{\beta}^{J}\right\}=2 \mathrm{i} \delta^{I J}\left(\gamma^{c}\right)_{\alpha \beta} P_{c}, \quad\left[Q_{\alpha}^{I}, P_{b}\right]=0, \\
& {\left[M_{\alpha \beta}, Q_{\gamma}^{I}\right]=\varepsilon_{\gamma(\alpha} Q_{\beta)}^{I}, \quad\left[\mathbb{D}, Q_{\alpha}^{I}\right]=\frac{1}{2} Q_{\alpha}^{I}, \quad\left[N_{K L}, Q_{\alpha}^{I}\right]=2 \delta_{[K}^{I} Q_{\alpha L]},}
\end{aligned}
$$

while the operators $S_{\alpha}^{I}$ obey the algebra

$$
\begin{aligned}
& \left\{S_{\alpha}^{I}, S_{\beta}^{J}\right\}=2 \mathrm{i} \delta^{I J}\left(\gamma^{c}\right)_{\alpha \beta} K_{c}, \quad\left[S_{\alpha}^{I}, K_{b}\right]=0, \\
& {\left[M_{\alpha \beta}, S_{\gamma}^{I}\right]=\varepsilon_{\gamma(\alpha} S_{\beta)}^{I}, \quad\left[\mathbb{D}, S_{\alpha}^{I}\right]=-\frac{1}{2} S_{\alpha}^{I}, \quad\left[N_{K L}, S_{\alpha}^{I}\right]=2 \delta_{[K}^{I} S_{\alpha L]} .}
\end{aligned}
$$

In the supersymmetric case, the translation $\left(P_{a}\right)$ and special conformal $\left(K_{a}\right)$ generators are extended to $P_{A}=\left(P_{a}, Q_{\alpha}^{I}\right)$ and $K_{A}=\left(K_{a}, S_{\alpha}^{I}\right)$, respectively. The remainder of the algebra of $K_{A}$ with $P_{A}$ is given by

$$
\begin{aligned}
{\left[K_{a}, Q_{\alpha}^{I}\right] } & =-\mathrm{i}\left(\gamma_{a}\right)_{\alpha}{ }^{\beta} S_{\beta}^{I}, \quad\left[S_{\alpha}^{I}, P_{a}\right]=\mathrm{i}\left(\gamma_{a}\right)_{\alpha}{ }^{\beta} Q_{\beta}^{I}, \\
\left\{S_{\alpha}^{I}, Q_{\beta}^{J}\right\} & =2 \varepsilon_{\alpha \beta} \delta^{I J} \mathbb{D}-2 \delta^{I J} M_{\alpha \beta}-2 \varepsilon_{\alpha \beta} N^{I J} .
\end{aligned}
$$

The superspace geometry of $\mathcal{N}$-extended conformal supergravity is formulated in terms of the covariant derivatives of the form

$$
\nabla_{A}=\left(\nabla_{a}, \nabla_{\alpha}^{I}\right)=E_{A}{ }^{M} \partial_{M}-\frac{1}{2} \Omega_{A}^{b c} M_{b c}-\frac{1}{2} \Phi_{A}{ }^{P Q} N_{P Q}-B_{A} \mathbb{D}-\mathfrak{F}_{A}{ }^{B} K_{B}
$$

Here $\Omega_{A}^{b c}$ is the Lorentz connection, $\Phi_{A} P Q$ the $\mathrm{SO}(\mathcal{N})$ connection, $B_{A}$ the dilatation connection, and $\mathfrak{F}_{A}{ }^{B}$ the special superconformal connection. The graded commutation relations of $\nabla_{A}$ with the generators $M_{b c}, N_{P Q}, \mathbb{D}$ and $K_{B}$ are obtained from (5.1) by the replacement $P_{A} \rightarrow \nabla_{A}$. However the relations (5.1b) turn into

$$
\begin{aligned}
{\left[\nabla_{A}, \nabla_{B}\right\}=} & -\mathcal{T}_{A B}{ }^{C} \nabla_{C}-\frac{1}{2} \mathcal{R}(M)_{A B}{ }^{c d} M_{c d}-\frac{1}{2} \mathcal{R}(N)_{A B}{ }^{P Q} N_{P Q} \\
& -\mathcal{R}(\mathbb{D})_{A B} \mathbb{D}-\mathcal{R}(S)_{A B}{ }_{K}^{\gamma} S_{\gamma}^{K}-\mathcal{R}(K)_{A B}{ }^{c} K_{c} .
\end{aligned}
$$


To describe the off-shell conformal supergravity multiplet, the torsion and curvature tensors should obey certain $\mathcal{N}$-dependent covariant constraints given in [38]. The complete solutions to the constraints are derived in [38]. We will reproduce the $\mathcal{N}=1$ and $\mathcal{N}=2$ solutions below.

The generators $K_{A}=\left(K_{a}, S_{\alpha}^{I}\right)$ are used to define conformal primary superfields:

$$
K_{A} \Phi=0 .
$$

In accordance with (5.1d), if a superfield is annihilated by the $S$-supersymmetry generator, then it is necessarily primary,

$$
S_{\alpha}^{I} \Phi=0 \quad \Longrightarrow \quad K_{a} \Phi=0 .
$$

\section{$5.1 \mathcal{N}=1$ SCHS theories}

The algebra of $\mathcal{N}=1$ conformal covariant derivatives [38] is

$$
\begin{aligned}
\left\{\nabla_{\alpha}, \nabla_{\beta}\right\} & =2 \mathrm{i} \nabla_{\alpha \beta}, \\
{\left[\nabla_{a}, \nabla_{\beta}\right] } & =\frac{1}{4}\left(\gamma_{a}\right)_{\beta}{ }^{\gamma} W_{\gamma \delta \sigma} K^{\delta \sigma}, \\
{\left[\nabla_{a}, \nabla_{b}\right] } & =-\frac{\mathrm{i}}{8} \varepsilon_{a b c}\left(\gamma^{c}\right)^{\alpha \beta} \nabla_{\alpha} W_{\beta \gamma \delta} K^{\gamma \delta}-\frac{1}{4} \varepsilon_{a b c}\left(\gamma^{c}\right)^{\alpha \beta} W_{\alpha \beta \gamma} S^{\gamma} .
\end{aligned}
$$

It is written in terms of the $\mathcal{N}=1$ super Cotton tensor $W_{\alpha \beta \gamma}$ which is a primary superfield of dimension $5 / 2$,

$$
S_{\delta} W_{\alpha \beta \gamma}=0, \quad \mathbb{D} W_{\alpha \beta \gamma}=\frac{5}{2} W_{\alpha \beta \gamma},
$$

obeying the Bianchi identity

$$
\nabla^{\alpha} W_{\alpha \beta \gamma}=0 .
$$

The super Cotton tensor $W_{\alpha \beta \gamma}$ was originally introduced in [84].

Consider a real primary superfield $L$ of dimension +2 ,

$$
S_{\alpha} L=0, \quad \mathbb{D} L=2 L .
$$

Then the functional

$$
I=\int \mathrm{d}^{3 \mid 2} z E L, \quad E^{-1}=\operatorname{Ber}\left(E_{A}{ }^{M}\right)
$$

is locally superconformal. We will use this action principle to construct $\mathcal{N}=1$ locally superconformal higher-spin actions.

We now introduce SCHS gauge prepotentials by extending the definitions given in [18-20] to conformal superspace. Given a positive integer $n>0$, a real tensor superfield $H_{\alpha(n)}$ is said to be a SCHS gauge prepotential if (i) it is primary and of dimension $(1-n / 2)$,

$$
S_{\beta} H_{\alpha(n)}=0, \quad \mathbb{D} H_{\alpha(n)}=\left(1-\frac{n}{2}\right) H_{\alpha(n)} ;
$$

and (ii) it is defined modulo gauge transformations of the form

$$
\delta_{\Lambda} H_{\alpha(n)}=\mathrm{i}^{n} \nabla_{\left(\alpha_{1}\right.} \Lambda_{\left.\alpha_{2} \ldots \alpha_{n}\right)},
$$


with the gauge parameter $\Lambda_{\alpha(n-1)}$ being real but otherwise unconstrained. The dimension of $H_{\alpha(n)}$ is uniquely fixed by requiring $\Lambda_{\alpha(n-1)}$ and the right-hand side of (5.12) to be primary.

Let us first discuss the case $n=1$ corresponding to a superconformal vector multiplet. Associated with the prepotential $H_{\alpha}$ is the real spinor descendant

$$
\mathfrak{W}_{\alpha}(H)=-\frac{\mathrm{i}}{2} \nabla^{\beta} \nabla_{\alpha} H_{\beta},
$$

which proves to be gauge invariant,

$$
\delta_{\Lambda} \mathfrak{W}_{\alpha}=0
$$

and primary,

$$
S_{\beta} \mathfrak{W}_{\alpha}=0, \quad \mathbb{D} \mathfrak{W}_{\alpha}=\frac{3}{2} \mathfrak{W}_{\alpha} .
$$

The field strength (5.13) obeys the Bianchi identity

$$
\nabla^{\alpha} \mathfrak{W}_{\alpha}=0
$$

In general, this conservation equation is superconformal, for some primary spinor $\mathfrak{W}_{\alpha}$, if the dimension of $\mathfrak{W}_{\alpha}$ is equal to $3 / 2$. The Chern-Simons action

$$
S_{\mathrm{SCS}}[H]=-\frac{\mathrm{i}}{2} \int \mathrm{d}^{3 \mid 2} z E H^{\alpha} \mathfrak{W}_{\alpha}(H)
$$

has the following basic properties: (i) it is locally superconformal; and (ii) it is invariant under the gauge transformations (5.12) with $n=1$.

It turns out that some of the properties of the conformal vector supermultiplet $(n=1)$, given by eqs. (5.14)-(5.16), cannot be extended to $n>1$ in the case of an arbitrary curved background. So let us first consider a conformally flat superspace,

$$
W_{\alpha \beta \gamma}=0 .
$$

Then it follows from (5.6) that the conformally covariant derivatives $\nabla_{A}=\left(\nabla_{a}, \nabla_{\alpha}\right)$ obey the same graded commutation relations as the flat-superspace covariant derivatives. This allows us to use the flat-superspace results of [18] provided local superconformal invariance can be kept under control. We associate with the gauge prepotential $H_{\alpha(n)}$ the following linearised higher-spin super Cotton tensor

$$
\begin{aligned}
\mathfrak{W}_{\alpha_{1} \ldots \alpha_{n}}:= & \frac{1}{2^{n}} \sum_{j=0}^{\lfloor n / 2\rfloor}\left\{\left(\begin{array}{c}
n \\
2 j
\end{array}\right)\left(\square_{c}\right)^{j} \nabla_{\left(\alpha_{1}{ }^{\beta_{1}} \ldots \nabla_{\alpha_{n-2 j}} \beta_{n-2 j} H_{\left.\alpha_{n-2 j+1} \ldots \alpha_{n}\right) \beta_{1} \ldots \beta_{n-2 j}}\right.}\right. \\
& \left.-\frac{\mathrm{i}}{2}\left(\begin{array}{c}
n \\
2 j+1
\end{array}\right) \nabla^{2}\left(\square_{c}\right)^{j} \nabla_{\left(\alpha_{1}\right.}{ }^{\beta_{1}} \ldots \nabla_{\alpha_{n-2 j-1}} \beta_{n-2 j-1} H_{\left.\alpha_{n-2 j} \ldots \alpha_{n}\right) \beta_{1} \ldots \beta_{n-2 j-1}}\right\},
\end{aligned}
$$

where we have denoted $\nabla^{2}=\nabla^{\alpha} \nabla_{\alpha}$. Making use of (5.1) it may be shown that $\mathfrak{W}_{\alpha(n)}(H)$ has the following properties:

1. It is primary,

$$
S_{\beta} \mathfrak{W}_{\alpha(n)}=0, \quad \mathbb{D} \mathfrak{W}_{\alpha(n)}=\left(1+\frac{n}{2}\right) \mathfrak{W}_{\alpha(n)} .
$$


2. It is conserved,

$$
\nabla^{\beta} \mathfrak{W}_{\beta \alpha(n-1)}=0
$$

3. It is invariant under the gauge transformations (5.12),

$$
\delta_{\Lambda} \mathfrak{W}_{\alpha(n)}=0
$$

These properties imply that the Chern-Simons-type action

$$
S_{\mathrm{SCS}}^{(n)}[H]=-\frac{\mathrm{i}^{n}}{2^{\lfloor n / 2\rfloor+1}} \int \mathrm{d}^{3 \mid 2} z E H^{\alpha(n)} \mathfrak{W}_{\alpha(n)}(H)
$$

has the following fundamental properties: (i) it is locally superconformal; and (ii) it is invariant under the gauge transformations (5.12). It is worth pointing out the existence of an alternative representation for $\mathfrak{W}_{\alpha(n)}$ inspired by the flat-superspace construction of [18]. It is given by

$$
\mathfrak{W}_{\alpha_{1} \ldots \alpha_{n}}=\left(-\frac{\mathrm{i}}{2}\right)^{n} \nabla^{\beta_{1}} \nabla_{\alpha_{1}} \ldots \nabla^{\beta_{n}} \nabla_{\alpha_{n}} H_{\beta_{1} \ldots \beta_{n}}=\mathfrak{W}_{\left(\alpha_{1} \ldots \alpha_{n}\right)} .
$$

To conclude our $\mathcal{N}=1$ discussion, we remark that the off-shell formulations for massless and massive higher-spin $\mathcal{N}=1$ supermultiplets in Minkowski and anti-de Sitter backgrounds were constructed in $[19,20]$. These theories are realised in terms of the conformal gauge prepotentials $H_{\alpha(n)}$ in conjunction with certain compensating supermultiplets.

\section{$5.2 \mathcal{N}=2$ SCHS theories}

In the $\mathcal{N}=2$ case it is convenient to replace the real spinor covariant derivatives $\nabla_{\alpha}^{I}$ with complex ones,

$$
\nabla_{\alpha}=\frac{1}{\sqrt{2}}\left(\nabla_{\alpha}^{1}-\mathrm{i} \nabla_{\alpha}^{2}\right), \quad \bar{\nabla}_{\alpha}=-\frac{1}{\sqrt{2}}\left(\nabla_{\alpha}^{1}+\mathrm{i} \nabla_{\alpha}^{2}\right),
$$

which are eigenvectors,

$$
\left[J, \nabla_{\alpha}\right]=\nabla_{\alpha}, \quad\left[J, \bar{\nabla}_{\alpha}\right]=-\bar{\nabla}_{\alpha},
$$

of the $\mathrm{U}(1)$ generator $J$ defined by

$$
J:=-\frac{\mathrm{i}}{2} \varepsilon^{K L} N_{K L} .
$$

It is also useful to introduce the operators

$$
S_{\alpha}:=\frac{1}{\sqrt{2}}\left(S_{\alpha}^{1}+\mathrm{i} S_{\alpha}^{2}\right), \quad \bar{S}_{\alpha}:=\frac{1}{\sqrt{2}}\left(S_{\alpha}^{1}-\mathrm{i} S_{\alpha}^{2}\right),
$$

which have the properties

$$
\left[J, \bar{S}_{\alpha}\right]=\bar{S}_{\alpha}, \quad\left[J, S_{\alpha}\right]=-S_{\alpha}
$$


The graded commutation relations specific to the new basis are

$$
\begin{aligned}
\left\{S_{\alpha}, S_{\beta}\right\} & =0, \quad\left\{\bar{S}_{\alpha}, \bar{S}_{\beta}\right\}=0, & & \left\{S_{\alpha}, \bar{S}_{\beta}\right\}=2 \mathrm{i} K_{\alpha \beta}, \\
{\left[K_{a}, \nabla_{\alpha}\right] } & =-\mathrm{i}\left(\gamma_{a}\right)_{\alpha}{ }^{\beta} \bar{S}_{\beta}, & & {\left[K_{a}, \bar{\nabla}_{\alpha}\right]=\mathrm{i}\left(\gamma_{a}\right)_{\alpha}{ }^{\beta} S_{\beta}, } \\
{\left[\bar{S}_{\alpha}, \nabla_{a}\right] } & =\mathrm{i}\left(\gamma_{a}\right)_{\alpha}{ }^{\beta} \nabla_{\beta}, & {\left[S_{\alpha}, \nabla_{a}\right] } & =-\mathrm{i}\left(\gamma_{a}\right)_{\alpha}{ }^{\beta} \bar{\nabla}_{\beta}, \\
\left\{\bar{S}_{\alpha}, \nabla_{\beta}\right\} & =0, & & \left\{S_{\alpha}, \bar{\nabla}_{\beta}\right\}=0, \\
\left\{\bar{S}_{\alpha}, \bar{\nabla}_{\beta}\right\} & =-2 \varepsilon_{\alpha \beta} \mathbb{D}+2 M_{\alpha \beta}-2 \varepsilon_{\alpha \beta} J, & & \left\{S_{\alpha}, \nabla_{\beta}\right\}=2 \varepsilon_{\alpha \beta} \mathbb{D}-2 M_{\alpha \beta}-2 \varepsilon_{\alpha \beta} J .
\end{aligned}
$$

In the complex basis, the algebra of $\mathcal{N}=2$ covariant derivatives [38] is

$$
\begin{aligned}
\left\{\nabla_{\alpha}, \nabla_{\beta}\right\} & =0, \quad\left\{\bar{\nabla}_{\alpha}, \bar{\nabla}_{\beta}\right\}=0, \\
\left\{\nabla_{\alpha}, \bar{\nabla}_{\beta}\right\} & =-2 \mathrm{i} \nabla_{\alpha \beta}-\varepsilon_{\alpha \beta} W_{\gamma \delta} K^{\gamma \delta}, \\
{\left[\nabla_{a}, \nabla_{\beta}\right] } & =\frac{\mathrm{i}}{2}\left(\gamma_{a}\right)_{\beta}{ }^{\gamma} \nabla_{\gamma} W^{\alpha \delta} K_{\alpha \delta}-\left(\gamma_{a}\right)_{\beta \gamma} W^{\gamma \delta} \bar{S}_{\delta}, \\
{\left[\nabla_{a}, \nabla_{b}\right] } & =-\frac{\mathrm{i}}{8} \varepsilon_{a b c}\left(\gamma^{c}\right)^{\gamma \delta}\left(\mathrm{i}\left[\nabla_{\gamma}, \bar{\nabla}_{\delta}\right] W_{\alpha \beta} K^{\alpha \beta}+4 \bar{\nabla}_{\gamma} W_{\delta \beta} \bar{S}^{\beta}+4 \nabla_{\gamma} W_{\delta \beta} S^{\beta}-8 W_{\gamma \delta} J\right),
\end{aligned}
$$

where the $\mathcal{N}=2$ super Cotton tensor $W_{\alpha \beta}$ is a primary real superfield,

$$
S_{\gamma} W_{\alpha \beta}=0 \quad \Longleftrightarrow \quad \bar{S}_{\gamma} W_{\alpha \beta}=0, \quad \mathbb{D} W_{\alpha \beta}=2 W_{\alpha \beta},
$$

with the fundamental property

$$
\nabla^{\alpha} W_{\alpha \beta}=0 .
$$

In $\mathrm{SO}(2)$ superspace [83], the super Cotton tensor $W_{\alpha \beta}$ was introduced originally in [85].

Given an integer $n>0$, a real tensor superfield $H_{\alpha(n)}$ is said to be a superconformal gauge prepotential if (i) it is primary and of dimension $(-n / 2)$,

$$
S_{\beta} H_{\alpha(n)}=0 \quad \Longleftrightarrow \quad \bar{S}_{\beta} H_{\alpha(n)}=0, \quad \mathbb{D} H_{\alpha(n)}=-\frac{n}{2} H_{\alpha(n)} ;
$$

and (ii) it is defined modulo gauge transformations of the form

$$
\delta_{\Lambda} H_{\alpha(n)}=\bar{\nabla}_{\left(\alpha_{1}\right.} \Lambda_{\left.\alpha_{2} \ldots \alpha_{n}\right)}-(-1)^{n} \nabla_{\left(\alpha_{1}\right.} \bar{\Lambda}_{\left.\alpha_{2} \ldots \alpha_{n}\right)},
$$

where the gauge parameter $\Lambda_{\alpha(n-1)}$ is a primary complex superfield of $\mathrm{U}(1)$ charge +1 , that is, $J \Lambda_{\alpha(n-1)}=\Lambda_{\alpha(n-1)}$. The dimension of the gauge prepotential is uniquely fixed by requiring $H_{\alpha(n)}$ and $\Lambda_{\alpha(n-1)}$ to be primary.

In the remainder of this section we assume that the background curved superspace $\mathcal{M}^{3 \mid 4}$ is conformally flat,

$$
W_{\alpha \beta}=0 .
$$

Associated with the gauge prepotential $H_{\alpha(n)}$ is the following real descendant

$$
\begin{aligned}
\mathfrak{W}_{\alpha(n)}(H)= & \frac{1}{2^{n-1}} \sum_{j=0}^{\lfloor n / 2\rfloor}\left\{\left(\begin{array}{c}
n \\
2 j
\end{array}\right) \Delta\left(\square_{c}\right)^{j} \nabla_{\left(\alpha_{1}\right.} \beta_{1} \ldots \nabla_{\alpha_{n-2 j}}^{\beta_{n-2 j}} H_{\left.\alpha_{n-2 j+1} \ldots \alpha_{n}\right) \beta_{1} \ldots \beta_{n-2 j}}\right. \\
& \left.+\left(\begin{array}{c}
n \\
2 j+1
\end{array}\right) \Delta^{2}\left(\square_{c}\right)^{j} \nabla_{\left(\alpha_{1}\right.}{ }^{\beta_{1}} \ldots \nabla_{\alpha_{n-2 j-1}} \beta_{n-2 j-1} H_{\left.\alpha_{n-2 j} \ldots \alpha_{n}\right) \beta_{1} \ldots \beta_{n-2 j-1}}\right\},
\end{aligned}
$$


where $\Delta=\frac{i}{2} \nabla^{\alpha} \bar{\nabla}_{\alpha}$. This descendant proves to be primary,

$$
S_{\beta} \mathfrak{W}_{\alpha(n)}=0 \quad \Longleftrightarrow \quad \bar{S}_{\beta} \mathfrak{W}_{\alpha(n)}=0, \quad \mathbb{D}_{\mathfrak{W}_{\alpha(n)}}=\left(1+\frac{n}{2}\right) \mathfrak{W}_{\alpha(n)},
$$

and gauge invariant,

$$
\delta_{\Lambda} \mathfrak{W}_{\alpha(n)}=0 .
$$

Moreover, it obeys the conservation equation

$$
\nabla^{\beta} \mathfrak{W}_{\beta \alpha_{1} \ldots \alpha_{n-1}}=0 \quad \Longleftrightarrow \quad \bar{\nabla}^{\beta} \mathfrak{W}_{\beta \alpha_{1} \ldots \alpha_{n-1}}=0
$$

These properties imply that the action

$$
S_{\mathrm{SCS}}^{(n)}[H]=-\frac{\mathrm{i}^{n}}{2^{\lfloor n / 2\rfloor+1}} \int \mathrm{d}^{3 \mid 4} z E H^{\alpha(n)} \mathfrak{W}_{\alpha(n)}(H)
$$

is superconformal and invariant under the gauge transformations (5.35).

To conclude our $\mathcal{N}=2$ analysis, we remark that the off-shell formulations for massless and massive higher-spin $\mathcal{N}=2$ supermultiplets in Minkowski superspace, as well as in the $(1,1)$ and $(2,0)$ anti-de Sitter backgrounds were constructed in [21, 22, 86]. These theories are realised in terms of the conformal gauge prepotentials $H_{\alpha(n)}$ in conjunction with certain compensating supermultiplets.

\section{$5.3 \mathcal{N}=3$ SCHS gauge prepotentials}

We introduce $\mathcal{N}=3$ SCHS prepotentials $H_{\alpha(n)}$, with $n$ a positive integer, with the following properties: (i) it is primary and of dimension $-(1+n / 2)$,

$$
S_{\beta}^{J} H_{\alpha(n)}=0, \quad \mathbb{D} H_{\alpha(n)}=-\left(1+\frac{n}{2}\right) H_{\alpha(n)} ;
$$

and (ii) it is defined modulo gauge transformations of the form

$$
\delta_{\Lambda} H_{\alpha(n)}=\mathrm{i}^{n} \nabla_{\left(\alpha_{1}\right.}^{I} \Lambda_{\left.\alpha_{2} \ldots \alpha_{n}\right)}^{I},
$$

with the primary gauge parameter $\Lambda_{\alpha(n-1)}^{I}$ being real but otherwise unconstrained. In the right-hand side of (5.43), summation over $I$ is assumed. The prepotential $H_{\alpha}$ corresponds to linearised $\mathcal{N}=3$ conformal supergravity [38].

The $\mathcal{N}=3$ story is still incomplete since higher-spin super Cotton tensors are not yet known.

\section{SCHS theories in four dimensions}

In $\mathcal{N}=1$ conformal superspace [41] in four dimensions, the covariant derivatives $\nabla_{A}=$ $\left(\nabla_{a}, \nabla_{\alpha}, \bar{\nabla}^{\dot{\alpha}}\right)$ have the form

$$
\begin{aligned}
\nabla_{A} & =E_{A}{ }^{M} \partial_{M}-\frac{1}{2} \Omega_{A}^{b c} M_{b c}-\mathrm{i} \Phi_{A} Y-B_{A} \mathbb{D}-\mathfrak{F}_{A}^{B} K_{B} \\
& =E_{A}{ }^{M} \partial_{M}-\Omega_{A}{ }^{\beta \gamma} M_{\beta \gamma}-\bar{\Omega}_{A} \dot{\beta} \dot{\gamma} \bar{M}_{\dot{\beta} \dot{\gamma}}-\mathrm{i} \Phi_{A} Y-B_{A} \mathbb{D}-\mathfrak{F}_{A}{ }^{B} K_{B} .
\end{aligned}
$$


Here $\Omega_{A}^{b c}$ is the Lorentz connection, $\Phi_{A}$ the $\mathrm{U}(1)_{\mathrm{R}}$ connection, $B_{A}$ the dilatation connection, and $\mathfrak{F}_{A}{ }^{B}$ the special superconformal connection. Below we list the graded commutation relations for the $\mathcal{N}=1$ superconformal algebra $\mathfrak{s u}(2,2 \mid 1)$ following the conventions adopted in $[87,88]$, keeping in mind that (i) the translation generators $P_{A}=\left(P_{a}, Q_{\alpha}, \bar{Q}^{\dot{\alpha}}\right)$ are replaced with $\nabla_{A}$; and (ii) the graded commutator $\left[\nabla_{A}, \nabla_{B}\right\}$ differs from that obtained from $\left[P_{A}, P_{B}\right\}$ by torsion and curvature dependent terms,

$$
\left[\nabla_{A}, \nabla_{B}\right\}=-\mathcal{T}_{A B}{ }^{C} \nabla_{C}-\frac{1}{2} \mathcal{R}_{A B}{ }^{c d}(M) M_{c d}-\mathrm{i} \mathcal{R}_{A B}(Y) Y-\mathcal{R}_{A B}(\mathbb{D}) \mathbb{D}-\mathcal{R}_{A B}{ }^{C}(K) K_{C}
$$

The Lorentz generators $M_{a b}$ act on the covariant derivatives as

$$
\left[M_{a b}, \nabla_{c}\right]=2 \eta_{c[a} \nabla_{b]}, \quad\left[M_{a b}, \nabla_{\gamma}\right]=\left(\sigma_{a b}\right)_{\gamma}^{\delta} \nabla_{\delta}, \quad\left[M_{a b}, \bar{\nabla}^{\dot{\gamma}}\right]=\left(\tilde{\sigma}_{a b}\right)_{\dot{\delta}}^{\dot{\gamma}} \bar{\nabla}^{\dot{\delta}} .
$$

The Lorentz generators with spinor indices act on the spinor covariant derivatives

$$
\left[M_{\alpha \beta}, \nabla_{\gamma}\right]=\varepsilon_{\gamma(\alpha} \nabla_{\beta)}, \quad\left[\bar{M}_{\dot{\alpha} \dot{\beta}}, \bar{\nabla}_{\dot{\gamma}}\right]=\varepsilon_{\dot{\gamma}(\dot{\alpha}} \bar{\nabla}_{\dot{\beta})} .
$$

The $\mathrm{U}(1)_{\mathrm{R}}$ and dilatation generators obey

$$
\begin{array}{ll}
{\left[Y, \nabla_{\alpha}\right]=\nabla_{\alpha},} & {\left[Y, \bar{\nabla}^{\dot{\alpha}}\right]=-\bar{\nabla}^{\dot{\alpha}},} \\
{\left[\mathbb{D}, \nabla_{a}\right]=\nabla_{a},} & {\left[\mathbb{D}, \nabla_{\alpha}\right]=\frac{1}{2} \nabla_{\alpha}, \quad\left[\mathbb{D}, \bar{\nabla}^{\dot{\alpha}}\right]=\frac{1}{2} \bar{\nabla}^{\dot{\alpha}} .}
\end{array}
$$

The special superconformal generators $K^{A}=\left(K^{a}, S^{\alpha}, \bar{S}_{\dot{\alpha}}\right)$ transform in the obvious way under the Lorentz group,

$$
\left[M_{a b}, K_{c}\right]=2 \eta_{c[a} K_{b]}, \quad\left[M_{a b}, S^{\gamma}\right]=-\left(\sigma_{a b}\right)_{\beta}{ }^{\gamma} S^{\beta}, \quad\left[M_{a b}, \bar{S}_{\dot{\gamma}}\right]=-\left(\sigma_{a b}\right)^{\dot{\beta}} \dot{\gamma} \bar{S}_{\dot{\beta}},
$$

and carry opposite $\mathrm{U}(1)_{\mathrm{R}}$ and dilatation weight to $\nabla_{A}$ :

$$
\begin{array}{ll}
{\left[Y, S^{\alpha}\right]=-S^{\alpha},} & {\left[Y, \bar{S}_{\dot{\alpha}}\right]=\bar{S}_{\dot{\alpha}},} \\
{\left[\mathbb{D}, K_{a}\right]=-K_{a}, \quad\left[\mathbb{D}, S^{\alpha}\right]=-\frac{1}{2} S^{\alpha}, \quad\left[\mathbb{D}, \bar{S}_{\dot{\alpha}}\right]=-\frac{1}{2} \bar{S}_{\dot{\alpha}} .}
\end{array}
$$

Among themselves, the generators $K^{A}$ obey the algebra

$$
\left\{S^{\alpha}, \bar{S}_{\dot{\alpha}}\right\}=2 \mathrm{i}\left(\sigma^{a}\right)^{\alpha}{ }_{\dot{\alpha}} K_{a},
$$

with all the other (anti-)commutators vanishing. Finally, the algebra of $K^{A}$ with $\nabla_{B}$ is given by

$$
\begin{array}{rlrl}
{\left[K^{a}, \nabla_{b}\right]} & =2 \delta_{b}^{a} \mathbb{D}+2 M_{b}^{a}, & \\
\left\{S^{\alpha}, \nabla_{\beta}\right\} & =2 \delta_{\beta}^{\alpha} \mathbb{D}-4 M^{\alpha}{ }_{\beta}-3 \delta_{\beta}^{\alpha} Y, & & \\
\left\{\bar{S}_{\dot{\alpha}}, \bar{\nabla}^{\dot{\beta}}\right\} & =2 \delta_{\dot{\alpha}}^{\dot{\beta}} \mathbb{D}+4 \bar{M}_{\dot{\alpha}}^{\dot{\beta}}+3 \delta_{\dot{\alpha}}^{\dot{\beta}} Y, & & \\
{\left[K^{a}, \nabla_{\beta}\right]} & =-\mathrm{i}\left(\sigma^{a}\right)_{\beta}{ }_{\beta} \bar{S}_{\dot{\beta}}, & & {\left[K^{a}, \bar{\nabla}^{\dot{\beta}}\right]=-\mathrm{i}\left(\sigma^{a}\right)^{\dot{\beta}}{ }_{\beta} S^{\beta},} \\
{\left[S^{\alpha}, \nabla_{b}\right]} & =\mathrm{i}\left(\sigma_{b}\right)^{\alpha}{ }_{\dot{\beta}} \bar{\nabla}^{\dot{\beta}}, & & {\left[\bar{S}_{\dot{\alpha}}, \nabla_{b}\right]=\mathrm{i}\left(\sigma_{b}\right)_{\dot{\alpha}}{ }^{\beta} \nabla_{\beta},}
\end{array}
$$

where all other graded commutators vanish. 
In conformal superspace [41], the torsion and curvature tensors in (6.2) are subject to covariant constraints such that $\left[\nabla_{A}, \nabla_{B}\right\}$ is expressed in terms of the super Weyl tensor $W_{\alpha \beta \gamma}=W_{(\alpha \beta \gamma)}$, its conjugate $\bar{W}_{\dot{\alpha} \dot{\beta} \dot{\gamma}}$ and their covariant derivatives. The solutions to the constraints are given by

$$
\begin{aligned}
\left\{\nabla_{\alpha}, \nabla_{\beta}\right\} & =\left\{\bar{\nabla}_{\dot{\alpha}}, \bar{\nabla}_{\dot{\beta}}\right\}=0, \quad \nabla_{\alpha \dot{\alpha}}:=\frac{\mathrm{i}}{2}\left\{\nabla_{\alpha}, \bar{\nabla}_{\dot{\alpha}}\right\} \\
{\left[\nabla_{\beta}, \nabla_{\alpha \dot{\alpha}}\right] } & =2 \mathrm{i} \varepsilon_{\beta \alpha} \bar{W}_{\dot{\alpha} \dot{\beta} \dot{\gamma}} \bar{M}^{\dot{\beta} \dot{\gamma}}-R(\bar{S})_{\beta \alpha \dot{\alpha} \dot{\gamma}} \bar{S}^{\dot{\gamma}}-R(K)_{\beta \alpha \dot{\alpha}}{ }^{c} K_{c},
\end{aligned}
$$

where $R(\bar{S})_{\beta \alpha \dot{\alpha} \dot{\gamma}}$ and $R(K)_{\beta \alpha \dot{\alpha}}{ }^{c}$ involve derivatives of the superfield $\bar{W}_{\dot{\alpha} \dot{\beta} \dot{\gamma}}$. Their precise expressions will not be necessary for our discussion; they can be found in the original publication [41].

Consider a primary superfield $\Psi$ (with suppressed indices), $K_{B} \Psi=0$. Its dimension $\Delta$ and $\mathrm{U}(1)_{\mathrm{R}}$ charge $q$ are defined as $\mathbb{D} \Psi=\Delta \Psi$ and $Y \Psi=q \Psi$. As is well known, for every primary covariantly chiral superfield $\phi_{\alpha(n)}$, its $\mathrm{U}(1)_{\mathrm{R}}$ charge is determined in terms of its dimension,

$$
K_{B} \phi_{\alpha(n)}=0, \quad \bar{\nabla}_{\dot{\beta}} \phi_{\alpha(n)}=0 \quad \Longrightarrow \quad q=-\frac{2}{3} \Delta .
$$

The super Weyl tensor $W_{\alpha \beta \gamma}$ is a primary chiral superfield of dimension $3 / 2$,

$$
K_{B} W_{\alpha \beta \gamma}=0, \quad \bar{\nabla}_{\dot{\beta}} W_{\alpha \beta \gamma}=0, \quad \mathbb{D} W_{\alpha \beta \gamma}=\frac{3}{2} W_{\alpha \beta \gamma} .
$$

It obeys the Bianchi identity

$$
B_{\alpha \dot{\alpha}}:=\mathrm{i} \nabla_{\dot{\alpha}}^{\beta} \nabla^{\gamma} W_{\alpha \beta \gamma}=\mathrm{i} \nabla_{\alpha}{ }^{\dot{\beta}} \bar{\nabla}^{\dot{\gamma}} \bar{W}_{\dot{\alpha} \dot{\beta} \dot{\gamma}}=\bar{B}_{\alpha \dot{\alpha}}
$$

Upon degauging (see [41] for the technical details of the degauging procedure) $B_{\alpha \dot{\alpha}}$ takes the form given in [32,33] (see also [17]). It is clear that $B_{\alpha \dot{\alpha}}$ is the $\mathcal{N}=1$ supersymmetric generalisation of the Bach tensor (4.42). One may check that $B_{\alpha \dot{\alpha}}$ is primary,

$$
K_{B} B_{\alpha \dot{\alpha}}=0, \quad \mathbb{D} B_{\alpha \dot{\alpha}}=3 B_{\alpha \dot{\alpha}},
$$

and obeys the conservation equation

$$
\nabla^{\alpha} B_{\alpha \dot{\alpha}}=0 \quad \Longleftrightarrow \quad \bar{\nabla}^{\dot{\alpha}} B_{\alpha \dot{\alpha}}=0
$$

The super-Bach tensor defined by eq. (6.8) naturally originates (see [32, 33] for the technical details) as a functional derivative of the conformal supergravity action ${ }^{14}[89,90]$,

$$
I_{\mathrm{CSG}}=\int \mathrm{d}^{4} x \mathrm{~d}^{2} \theta \mathcal{E} W^{\alpha \beta \gamma} W_{\alpha \beta \gamma}+\text { c.c. },
$$

with respect to the gravitational superfield $H^{\alpha \dot{\alpha}}[89]$, specifically

$$
\delta \int \mathrm{d}^{4} x \mathrm{~d}^{2} \theta \mathcal{E} W^{\alpha \beta \gamma} W_{\alpha \beta \gamma}=\int \mathrm{d}^{4} x \mathrm{~d}^{2} \theta \mathrm{d}^{2} \bar{\theta} E \Delta H^{\alpha \dot{\alpha}} B_{\alpha \dot{\alpha}},
$$

\footnotetext{
${ }^{14}$ In Minkowski superspace, the linearised action for conformal supergravity was constructed by Ferrara and Zumino [34].
} 
where $\mathcal{E}$ denotes the chiral integration measure, and $\Delta H^{\alpha \dot{\alpha}}$ the covariant variation of the gravitational superfield defined in [91, 92]. The conservation equation (6.10) expresses the gauge invariance of the conformal supergravity action.

We introduce SCHS gauge prepotentials by generalising the construction of [17]. Given two positive integers $m$ and $n$, a SCHS gauge prepotential $\Upsilon_{\alpha(m) \dot{\alpha}(n)}$ is a primary superfield, $K_{B} \Upsilon_{\alpha(m) \dot{\alpha}(n)}=0$, defined modulo gauge transformations [17]

$$
\delta_{\Lambda, \zeta} \Upsilon_{\alpha_{1} \ldots \alpha_{m} \dot{\alpha}_{1} \ldots \dot{\alpha}_{n}}=\bar{\nabla}_{\left(\dot{\alpha}_{1}\right.} \Lambda_{\left.\alpha_{1} \ldots \alpha_{m} \dot{\alpha}_{2} \ldots \dot{\alpha}_{n}\right)}+\nabla_{\left(\alpha_{1}\right.} \zeta_{\left.\alpha_{2} \ldots \alpha_{m}\right) \dot{\alpha}_{1} \ldots \dot{\alpha}_{n}}
$$

with unconstrained primary gauge parameters $\Lambda_{\alpha(m) \dot{\alpha}(n-1)}$ and $\zeta_{\alpha(m-1) \dot{\alpha}(n)}$. The conditions that $\Upsilon_{\alpha(m) \dot{\alpha}(n)}, \Lambda_{\alpha(m) \dot{\alpha}(n-1)}$ and $\zeta_{\alpha(m-1) \dot{\alpha}(n)}$ be primary superfields uniquely fix the dimension and $\mathrm{U}(1)_{\mathrm{R}}$ charge of $\Upsilon_{\alpha(m) \dot{\alpha}(n)}$,

$$
\mathbb{D} \Upsilon_{\alpha(m) \dot{\alpha}(n)}=-\frac{1}{2}(m+n) \Upsilon_{\alpha(m) \dot{\alpha}(n)}, \quad Y \Upsilon_{\alpha(m) \dot{\alpha}(n)}=\frac{1}{3}(m-n) \Upsilon_{\alpha(m) \dot{\alpha}(n)} .
$$

Associated with $\Upsilon_{\alpha(m) \dot{\alpha}(n)}$ and its conjugate $\bar{\Upsilon}_{\alpha(n) \dot{\alpha}(m)}$ are higher-derivative descendants

$$
\begin{aligned}
& \hat{\mathfrak{W}}_{\alpha(m+n+1)}:=-\frac{1}{4} \bar{\nabla}^{2} \nabla_{\left(\alpha_{1}\right.} \dot{\beta}_{1} \ldots \nabla_{\alpha_{n}}{ }^{\dot{\beta}_{n}} \nabla_{\alpha_{n+1}} \Upsilon_{\left.\alpha_{n+2} \ldots \alpha_{m+n+1}\right) \dot{\beta}_{1} \ldots \dot{\beta}_{n}} \\
& \check{\mathfrak{W}}_{\alpha(m+n+1)}:=-\frac{1}{4} \bar{\nabla}^{2} \nabla_{\left(\alpha_{1}\right.} \dot{\beta}_{1} \cdots \nabla_{\alpha_{m}}{ }^{\dot{\beta}_{m}} \nabla_{\alpha_{m+1}} \bar{\Upsilon}_{\left.\alpha_{m+2} \ldots \alpha_{m+n+1}\right) \dot{\beta}_{1} \ldots \dot{\beta}_{m}} .
\end{aligned}
$$

By construction they are obviously covariantly chiral,

$$
\bar{\nabla}_{\dot{\beta}} \hat{\mathfrak{W}}_{\alpha(m+n+1)}=0, \quad \bar{\nabla}_{\dot{\beta}} \check{\mathfrak{W}}_{\alpha(m+n+1)}=0 .
$$

What is less trivial is the fact that they are primary,

$$
\begin{aligned}
K_{B} \hat{\mathfrak{W}}_{\alpha(m+n+1)}=0, & \mathbb{D} \hat{\mathfrak{W}}_{\alpha(m+n+1)}=\frac{1}{2}(3+n-m) \hat{\mathfrak{W}}_{\alpha(m+n+1)}, \\
K_{B} \check{\mathfrak{W}}_{\alpha(m+n+1)}=0, & \mathbb{D} \check{\mathfrak{W}}_{\alpha(m+n+1)}=\frac{1}{2}(3+m-n) \check{\mathfrak{W}}_{\alpha(m+n+1)} .
\end{aligned}
$$

These properties imply that the following action

$$
S_{\mathrm{SCHS}}^{(m, n)}=\mathrm{i}^{m+n} \int \mathrm{d}^{4} x \mathrm{~d}^{2} \theta \mathcal{E} \hat{\mathfrak{W}}^{\alpha_{1} \ldots \alpha_{m+n+1}} \check{\mathfrak{W}}_{\alpha_{1} \ldots \alpha_{m+n+1}}+\text { c.c. }
$$

is locally superconformal.

Consider a conformally flat background superspace,

$$
W_{\alpha \beta \gamma}=0 .
$$

Then it may be shown that the chiral descendants (6.15) are invariant under the gauge transformations (6.13),

$$
\delta_{\Lambda, \zeta} \hat{\mathfrak{W}}_{\alpha(m+n+1)}=0, \quad \delta_{\Lambda, \zeta \mathfrak{W}_{\alpha(m+n+1)}}=0 .
$$

As a result, the higher-spin actions (6.18) are gauge invariant. It is clear that these actions are modelled on the conformal supergravity action. 
There are several special cases that require a separate discussion. Firstly, choosing $m=n=s>0$ allows us to impose the reality condition

$$
\Upsilon_{\alpha(s) \dot{\alpha}(s)}=\bar{\Upsilon}_{\alpha(s) \dot{\alpha}(s)} \equiv H_{\alpha(s) \dot{\alpha}(s)},
$$

and then (6.13) turns into the transformation law [17]

$$
\delta_{\Lambda} H_{\alpha_{1} \ldots \alpha_{s} \dot{\alpha}_{1} \ldots \dot{\alpha}_{s}}=\bar{\nabla}_{\left(\dot{\alpha}_{1}\right.} \Lambda_{\left.\alpha_{1} \ldots \alpha_{s} \dot{\alpha}_{2} \ldots \dot{\alpha}_{s}\right)}-\nabla_{\left(\alpha_{1}\right.} \bar{\Lambda}_{\left.\alpha_{2} \ldots \alpha_{s}\right) \dot{\alpha}_{1} \ldots \dot{\alpha}_{s}}
$$

which is a curved-superspace extension of (1.8). The gauge prepotential $H_{\alpha(s) \dot{\alpha}(s)}$ describes the conformal superspin- $\left(s+\frac{1}{2}\right)$ multiplet, with the lowest choice $s=1$ corresponding to linearised conformal supergravity. It is one of the dynamical variables in terms of which the off-shell massless superspin- $\left(s+\frac{1}{2}\right)$ multiplets in Minkowski and anti-de Sitter backgrounds are formulated [93, 94].

The second special case corresponds to $m=n+1=s>1$. The gauge prepotential $\Upsilon_{\alpha(s) \dot{\alpha}(s-1)}$ and its conjugate, along with certain compensating supermultiplets, are used to describe the off-shell massless superspin- $s$ multiplet in Minkowski and anti-de Sitter backgrounds, originally proposed in [94, 95] and recently reformulated in [96, 97].

Thirdly, the case $m=1$ and $n=0$, which corresponds to the superconformal gravitino multiplet, has been excluded from the previous consideration since the transformation law (6.13) is not defined. This supermultiplet is characterised by the gauge freedom [17]

$$
\delta \Upsilon_{\alpha}=\nabla_{\alpha} \zeta+\lambda_{\alpha}, \quad \bar{\nabla}_{\dot{\beta}} \lambda_{\alpha}=0
$$

which is a curved superspace extension of the transformation law given by Gates and Siegel [98] who studied an off-shell formulation for the massless gravitino supermultiplet in Minkowski superspace.

\section{Concluding comments}

There exist two modern approaches to formulate conformal geometry. One of them was developed by mathematicians and is often referred to as tractor calculus [99, 100], with its roots going back to the work of Thomas [101]. The other formalism was created by supergravity practitioners [30]. It describes conformal gravity as the gauge theory of the conformal group, which was reviewed in section 2. It may be shown that the former approach is obtained from the latter by imposing the gauge condition (2.25), which makes transparent the fact that the $\mathfrak{s o}(D, 2)$ connection (2.18) encodes the tractor connection of $[99,100]$. This means that the two approaches to conformal geometry are essentially equivalent and complementary.

Tractor calculus has been used to construct families of conformal differential operators. Moreover, there have appeared interesting applications of this formalism in physics, see [102-106] and references therein. At the same time, tractor calculus is not practical if one is interested in constructing superconformal field theories, and alternative ideas are required. Fortunately, the work of Butter in four dimensions [41, 42] and its extensions to three, five and six dimensions $[38,39,43,44]$ have provided powerful tools to describe 
conformal supergravity and its higher-order invariants in superspace. ${ }^{15}$ In this paper we have demonstrated the power of conformal (super)space to generate (super) CHS theories.

In four dimensions, all of the higher-spin models which have been constructed in this paper are (super) Weyl-invariant on general curved backgrounds. However, their higherspin gauge invariance is present, in general, only for conformally flat backgrounds. In principle, extensions to more general (Bach-flat) backgrounds are possible by deforming the action with non-minimal primary terms containing factors of the (super) Weyl tensor and its covariant derivatives. Instructive examples are provided by (i) the model (4.39) with $\omega=-1$; (ii) the conformal gravitino model considered in appendix E; (iii) the conformal graviton model considered in appendix F; and (iv) the superconformal gravitino model studied in [17]. Conformal (super)space is an ideal formalism for constructing such deformations since the algebra of covariant derivatives is determined by the (super) Weyl tensor and its covariant derivatives.

The structure of the (super) CHS actions presented in this paper indicate that there should exist a generating formalism to formulate all of these models in terms of a single hyper-action. Recently there has been much interest in the so-called tensorial or hyperspace approach to the description of massless higher-spin (super)fields [108-122], see also [123] for a pedagogical review. It would be interesting to study whether the conformal (super)space methods can be extended to hyperspace.

\section{Acknowledgments}

SMK acknowledges email correspondence with Daniel Butter, Dmitri Sorokin and especially Arkady Tseytlin. MP is grateful to Gabriele Tartaglino-Mazzucchelli for illuminating discussions. The work of SMK is supported in part by the Australian Research Council, project No. DP160103633. The work of MP is supported by the Hackett Postgraduate Scholarship UWA, under the Australian Government Research Training Program.

\section{A 3D notation and conventions}

In $3 D$ we follow the notation and conventions adopted in [83]. In particular, the Minkowski metric is $\eta_{a b}=\operatorname{diag}(-1,1,1)$. The spinor indices are raised and lowered using the $\operatorname{SL}(2, \mathbb{R})$ invariant tensors

$$
\varepsilon_{\alpha \beta}=\left(\begin{array}{cc}
0 & -1 \\
1 & 0
\end{array}\right), \quad \varepsilon^{\alpha \beta}=\left(\begin{array}{cc}
0 & 1 \\
-1 & 0
\end{array}\right), \quad \varepsilon^{\alpha \gamma} \varepsilon_{\gamma \beta}=\delta_{\beta}^{\alpha}
$$

by the standard rule:

$$
\psi^{\alpha}=\varepsilon^{\alpha \beta} \psi_{\beta}, \quad \psi_{\alpha}=\varepsilon_{\alpha \beta} \psi^{\beta} .
$$

We make use of real gamma-matrices, $\gamma_{a}:=\left(\left(\gamma_{a}\right)_{\alpha}{ }^{\beta}\right)$, which obey the algebra

$$
\gamma_{a} \gamma_{b}=\eta_{a b} \mathbb{1}+\varepsilon_{a b c} \gamma^{c},
$$

\footnotetext{
${ }^{15}$ The conformal superspace approach is at the heart of the construction of all $\mathcal{N}=4$ conformal supergravity theories in four dimensions [107].
} 
where the Levi-Civita tensor is normalised as $\varepsilon^{012}=-\varepsilon_{012}=1$. The completeness relation for the gamma-matrices reads

$$
\left(\gamma^{a}\right)_{\alpha \beta}\left(\gamma_{a}\right)^{\rho \sigma}=-\left(\delta_{\alpha}^{\rho} \delta_{\beta}^{\sigma}+\delta_{\alpha}^{\sigma} \delta_{\beta}^{\rho}\right)
$$

Here the symmetric matrices $\left(\gamma_{a}\right)^{\alpha \beta}$ and $\left(\gamma_{a}\right)_{\alpha \beta}$ are obtained from $\left(\gamma_{a}\right)_{\alpha}{ }^{\beta}$ by the rules (A.2). Some useful relations involving $\gamma$-matrices are

$$
\begin{aligned}
\varepsilon_{a b c}\left(\gamma^{b}\right)_{\alpha \beta}\left(\gamma^{c}\right)_{\gamma \delta} & =\varepsilon_{\gamma(\alpha}\left(\gamma_{a}\right)_{\beta) \delta}+\varepsilon_{\delta(\alpha}\left(\gamma_{a}\right)_{\beta) \gamma}, \\
\operatorname{tr}\left[\gamma_{a} \gamma_{b} \gamma_{c} \gamma_{d}\right] & =2 \eta_{a b} \eta_{c d}-2 \eta_{a c} \eta_{d b}+2 \eta_{a d} \eta_{b c} .
\end{aligned}
$$

Given a three-vector $x_{a}$, it can be equivalently described by a symmetric second-rank spinor $x_{\alpha \beta}$ defined as

$$
x_{\alpha \beta}:=\left(\gamma^{a}\right)_{\alpha \beta} x_{a}=x_{\beta \alpha}, \quad x_{a}=-\frac{1}{2}\left(\gamma_{a}\right)^{\alpha \beta} x_{\alpha \beta} .
$$

In the $3 D$ case, an antisymmetric tensor $F_{a b}=-F_{b a}$ is Hodge-dual to a three-vector $F_{a}$, specifically

$$
F_{a}=\frac{1}{2} \varepsilon_{a b c} F^{b c}, \quad F_{a b}=-\varepsilon_{a b c} F^{c} .
$$

Then, the symmetric spinor $F_{\alpha \beta}=F_{\beta \alpha}$, which is associated with $F_{a}$, can equivalently be defined in terms of $F_{a b}$ :

$$
F_{\alpha \beta}:=\left(\gamma^{a}\right)_{\alpha \beta} F_{a}=\frac{1}{2}\left(\gamma^{a}\right)_{\alpha \beta} \varepsilon_{a b c} F^{b c} .
$$

These three algebraic objects, $F_{a}, F_{a b}$ and $F_{\alpha \beta}$, are in one-to-one correspondence with each other, $F_{a} \leftrightarrow F_{a b} \leftrightarrow F_{\alpha \beta}$. The corresponding inner products are related to each other as follows:

$$
-F^{a} G_{a}=\frac{1}{2} F^{a b} G_{a b}=\frac{1}{2} F^{\alpha \beta} G_{\alpha \beta} .
$$

The Lorentz generators with two vector indices $\left(M_{a b}=-M_{b a}\right)$, one vector index $\left(M_{a}\right)$ and two spinor indices $\left(M_{\alpha \beta}=M_{\beta \alpha}\right)$ are related to each other by the rules: $M_{a}=\frac{1}{2} \varepsilon_{a b c} M^{b c}$ and $M_{\alpha \beta}=\left(\gamma^{a}\right)_{\alpha \beta} M_{a}$. These generators act on a vector $V_{c}$ and a spinor $\Psi_{\gamma}$ as follows:

$$
M_{a b} V_{c}=2 \eta_{c[a} V_{b]}, \quad M_{\alpha \beta} \Psi_{\gamma}=\varepsilon_{\gamma(\alpha} \Psi_{\beta)} .
$$

The $D=3$ conformal algebra in spinor notation is

$$
\begin{aligned}
{\left[M_{\alpha \beta}, M_{\gamma \delta}\right] } & =\varepsilon_{\gamma(\alpha} M_{\beta) \delta}+\varepsilon_{\delta(\alpha} M_{\beta) \gamma}, & & \\
{\left[M_{\alpha \beta}, P_{\gamma \delta}\right] } & =\varepsilon_{\gamma(\alpha} P_{\beta) \delta}+\varepsilon_{\delta(\alpha} P_{\beta) \gamma}, & & {\left[\mathbb{D}, P_{\alpha \beta}\right]=P_{\alpha \beta}, } \\
{\left[M_{\alpha \beta}, K_{\gamma \delta}\right] } & =\varepsilon_{\gamma(\alpha} K_{\beta) \delta}+\varepsilon_{\delta(\alpha} K_{\beta) \gamma}, & & {\left[\mathbb{D}, K_{\alpha \beta}\right]=-K_{\alpha \beta}, } \\
{\left[K_{\alpha \beta}, P_{\gamma \delta}\right] } & =4 \varepsilon_{\gamma(\alpha} \varepsilon_{\beta) \delta} \mathbb{D}-4 \varepsilon_{(\gamma(\alpha} M_{\beta) \delta)}, & &
\end{aligned}
$$

where $M_{\alpha \beta}=\left(\gamma^{a}\right)_{\alpha \beta} M_{a}, P_{\alpha \beta}=\left(\gamma^{a}\right)_{\alpha \beta} P_{a}$ and $K_{\alpha \beta}=\left(\gamma^{a}\right)_{\alpha \beta} K_{a}$.

To describe conformal gravity in three dimensions we made use of the symmetric Cartan-Killing metric on $\mathfrak{s o}(3,2), \Gamma_{\tilde{a} \tilde{b}}=f_{\tilde{a} \tilde{d}} \tilde{c} \tilde{b}_{\tilde{b} \tilde{c}}^{\tilde{d}}$, see [39] for the technical details. In accordance with (2.3), the non-vanishing components of $\Gamma_{\tilde{a} \tilde{b}}$ are

$$
\Gamma_{M_{a b}, M_{c d}}=-12 \eta_{a[c} \eta_{d] b}, \quad \Gamma_{K_{a}, P_{b}}=-12 \eta_{a b}, \quad \Gamma_{\mathbb{D D}}=6 .
$$




\section{B Converting between conventions}

In several papers such as ref. [20], a different set of conventions were used. The purpose of this appendix is to provide a summary of how to easily convert between conventions.

Suppose that a field $\Phi$ transforms under some tensor representation of the gauge group $\mathcal{G}$. We may write the action of the generators $X_{\tilde{a}}$ on $\Phi$ as

$$
X_{\tilde{a}} \Phi=t_{\tilde{a}} \Phi
$$

for some matrix $t_{\tilde{a}}$. Since the operator $X_{\tilde{a}}$ passes through $t_{\tilde{b}}$, we find that the commutator is

$$
\left[X_{\tilde{a}}, X_{\tilde{b}}\right] \Phi=-\left[t_{\tilde{a}}, t_{\tilde{b}}\right] \Phi
$$

The $X_{\tilde{a}}$ and $t_{\tilde{a}}$ therefore satisfy

$$
\left[X_{\tilde{a}}, X_{\tilde{b}}\right]=-f_{\tilde{a} \tilde{b}}^{\tilde{c}} X_{\tilde{c}}, \quad\left[t_{\tilde{a}}, t_{\tilde{b}}\right]=+f_{\tilde{a} \tilde{b}} \tilde{c} t_{\tilde{c}}
$$

In particular, the action of the Lorentz generators is $M_{a b} \Phi=m_{a b} \Phi$. The Lorentz generators of ref. [20] correspond to $m_{a b}$, i.e. no change is necessary here.

In section 2, the connection one-forms, torsion and curvature tensors were defined through

$$
\nabla_{a}=e_{a}-\omega_{a}^{\underline{b}} X_{\underline{b}}, \quad\left[\nabla_{a}, \nabla_{b}\right]=-\mathcal{T}_{a b}^{c} \nabla_{c}-\mathcal{R}_{a b} \underline{c}_{\underline{c}}
$$

which differs to the definitions in refs. [20] and [33] by a minus sign. Thus, to flow between conventions, one must impose the gauge $\mathfrak{b}_{a}=0$ and make the following replacements

$$
\begin{aligned}
T_{a b}{ }^{c} & \longmapsto-T_{a b}{ }^{c}, \\
R_{a b c d} & \longmapsto-R_{a b c d}, \\
\omega_{a b c} & \longmapsto-\omega_{a b c} .
\end{aligned}
$$

Additionally, since the Cotton, Weyl and Schouten tensors, given by (2.45), (2.34) and (2.32) respectively, are defined in terms of $R_{a b c d}$, we must also rescale each of them by -1 . This accounts for the sign discrepancy between the second and third terms of $(2.62 \mathrm{~b})$ and the first two terms of $(3.5 \mathrm{c})$.

\section{Properties of the generalised HS Cotton tensor}

In this section we present the main steps that are needed in order to prove the two properties (3.22) of the generalised higher-spin Cotton tensor $\mathfrak{C}_{\alpha(n)}^{(l)}$. Namely, that in any conformally flat spacetime it is partially conserved and gauge invariant. 
It may be shown that the $l^{\text {th }}$ divergence of $\mathfrak{C}_{\alpha(n)}^{(l)}$ is given by

$$
\begin{aligned}
& 2^{n-2 l+1} \nabla^{\beta_{1} \beta_{2}} \ldots \nabla^{\beta_{2 l-1} \beta_{2 l}} \mathfrak{C}_{\alpha(n-2 l) \beta(2 l)}^{(l)} \\
& =\sum_{j=l-1}^{\left\lceil\frac{n}{2}\right\rceil-1}\left(\begin{array}{c}
n \\
2 j+1
\end{array}\right)\left(\begin{array}{c}
j \\
l-1
\end{array}\right)\left(\square_{c}\right)^{j-l+1} \nabla^{\beta_{1} \beta_{2}} \cdots \nabla^{\beta_{2 l-1} \beta_{2 l}} \\
& \times \frac{1}{n !} \sum_{k=0}^{l}\left(\begin{array}{l}
l \\
k
\end{array}\right)\left(\begin{array}{c}
n-2 j-1 \\
2 k
\end{array}\right)(2 k) !\left(\begin{array}{c}
n-2 l \\
n-2 j-2 k-1
\end{array}\right)(n-2 j-2 k-1) !(2 j+1) ! \\
& \times \nabla_{\beta_{1}}{ }^{\gamma_{1}} \cdots \nabla_{\beta_{2 k}}^{\gamma_{2 k}} \nabla_{\left(\alpha_{1}\right.} \gamma_{2 k+1} \ldots \nabla_{\alpha_{n-2 j-2 k-1}}^{\gamma_{n-2 j-1}} h_{\left.\alpha_{n-2 j-2 k} \ldots \alpha_{n-2 l}\right) \beta_{2 k+1} \ldots \beta_{2 l} \gamma_{1} \ldots \gamma_{n-2 j-1}}^{(l)} \\
& =\sum_{k=0}^{l} \sum_{j=l-k}^{\left\lceil\frac{n}{2}\right\rceil-k-1}(-1)^{k}\left(\begin{array}{c}
n \\
2 j+1
\end{array}\right)\left(\begin{array}{c}
j \\
l-1
\end{array}\right)\left(\begin{array}{l}
l \\
k
\end{array}\right)\left(\begin{array}{c}
n-2 j-1 \\
2 k
\end{array}\right)\left(\begin{array}{c}
n-2 l \\
n-2 j-2 k-1
\end{array}\right) \frac{(2 k) !(2 j+1) !}{n !} \\
& \times(n-2 j-2 k-1) !\left(\square_{c}\right)^{j+k-l+1} \nabla^{\gamma_{1} \gamma_{2}} \cdots \nabla^{\gamma_{2 l-1} \gamma_{2 l}} \nabla_{\left(\alpha_{1}\right.}{ }^{\gamma_{2 l+1}} \cdots \nabla_{\alpha_{n-2 j-2 k-1}}{ }^{\gamma_{n-2 j-2 k+2 l-1}} \\
& \times h_{\left.\alpha_{n-2 j-2 k} \ldots \alpha_{n-2 l}\right) \gamma_{1} \ldots \gamma_{n-2 j-2 k+2 l-1}}^{(l)} \\
& =\sum_{j=l}^{\left\lceil\frac{n}{2}\right\rceil-1}\left(\begin{array}{c}
n-2 l \\
n-2 j-1
\end{array}\right)\left(\square_{c}\right)^{j-l+1} \nabla^{\gamma_{1} \gamma_{2}} \cdots \nabla^{\gamma_{2 l-1} \gamma_{2 l}} \nabla_{\left(\alpha_{1}\right.}^{\gamma_{2 l+1}} \cdots \nabla_{\alpha_{n-2 j-1}}^{\gamma_{n-2 j+2 l-1}} \\
& \times h_{\left.\alpha_{n-2 j} \ldots \alpha_{n-2 l}\right) \gamma_{1} \ldots \gamma_{n-2 j+2 l-1}}^{(l)}\left\{\sum_{k=0}^{l}(-1)^{k}\left(\begin{array}{l}
j-k \\
l-1
\end{array}\right)\left(\begin{array}{l}
l \\
k
\end{array}\right)\right\} .
\end{aligned}
$$

Making use of the combinatoric identity

$$
\sum_{k=0}^{l}(-1)^{k}\left(\begin{array}{l}
j-k \\
l-1
\end{array}\right)\left(\begin{array}{l}
l \\
k
\end{array}\right)=0 \quad \forall j \geq l,
$$

which may be proved by induction on $l$, it follows that the last line in the above is equal to zero. In the second line we have used the combinatoric factors to shift the upper and lower bounds of the summation over $j$. Then, in the third line we have shifted the dummy variable $j \mapsto j-k$.

Under the gauge transformations (3.16), it may be shown that $\mathfrak{C}_{\alpha(n)}^{(l)}$ transforms as

$$
\begin{aligned}
& \delta_{\xi}\left(2^{n-2 l+1} \mathfrak{C}_{\alpha(n)}^{(l)}\right) \\
& =\sum_{j=l-1}^{\left\lceil\frac{n}{2}\right\rceil-1}\left(\begin{array}{c}
n \\
2 j+1
\end{array}\right)\left(\begin{array}{c}
j \\
l-1
\end{array}\right)\left(\square_{c}\right)^{j-l+1} \nabla_{\left(\alpha_{1}\right.} \beta_{1} \cdots \nabla_{\alpha_{n-2 j-1}} \beta_{n-2 j-1} \\
& \quad \times \frac{1}{n !} \sum_{k=0}^{l}\left(\begin{array}{c}
n-2 j-1 \\
2 k
\end{array}\right)\left(\begin{array}{c}
l \\
k
\end{array}\right)(2 k) !\left(\begin{array}{c}
2 j+1 \\
2 l-2 k
\end{array}\right)(2 l-2 k) !(n-2 l) ! \\
& \quad \times \nabla_{\mid \beta_{1} \beta_{2}} \cdots \nabla_{\beta_{2 k-1} \beta_{2 k} \mid} \nabla_{\alpha_{n-2 j} \alpha_{n-2 j+1}} \cdots \nabla_{\alpha_{n-2 j-2 k+2 l-2} \alpha_{n-2 j-2 k+2 l-1}} \\
& \quad \times \xi_{\left.\alpha_{n-2 j-2 k+2 l} \ldots \alpha_{n}\right) \beta_{2 k+1} \ldots \beta_{n-2 j-1}}
\end{aligned}
$$




$$
\begin{aligned}
& =\sum_{k=0}^{l} \sum_{j=l-k}^{\left\lceil\frac{n}{2}\right\rceil-k-1}(-1)^{k}\left(\begin{array}{c}
n \\
2 j+1
\end{array}\right)\left(\begin{array}{c}
j \\
l-1
\end{array}\right)\left(\begin{array}{c}
n-2 j-1 \\
2 k
\end{array}\right)\left(\begin{array}{l}
l \\
k
\end{array}\right)\left(\begin{array}{c}
2 j+1 \\
2 l-2 k
\end{array}\right) \frac{(2 k) !(2 l-2 k) !(n-2 l) !}{n !} \\
& \quad \times\left(\square_{c}\right)^{j+k-l+1} \nabla_{\left(\alpha_{1} \alpha_{2}\right.} \cdots \nabla_{\alpha_{2 l-1} \alpha_{2 l}} \nabla_{\alpha_{2 l+1}} \beta_{2 l+1} \cdots \nabla_{\alpha_{n-2 j-2 k+2 l+1}} \beta_{n-2 j-2 k+2 l+1} \\
& \quad \times \xi_{\left.\alpha_{n-2 j-2 k+2 l+2} \ldots \alpha_{n}\right) \beta_{2 l+1} \ldots \beta_{n-2 j-2 k+2 l+1}} \\
& =\sum_{j=l}^{\left\lceil\frac{n}{2}\right\rceil-1}\left(\begin{array}{c}
n-2 l \\
2 j-2 l+1
\end{array}\right)\left(\square_{c}\right)^{j-l+1} \nabla_{\left(\alpha_{1} \alpha_{2}\right.} \cdots \nabla_{\alpha_{2 l-1} \alpha_{2 l}} \nabla_{\alpha_{2 l+1}} \beta_{2 l+1} \cdots \nabla_{\alpha_{n-2 j+2 l+1}} \beta_{n-2 j+2 l+1} \\
& \quad \times \xi_{\left.\alpha_{n-2 j+2 l+2} \ldots \alpha_{n}\right) \beta_{2 l+1} \ldots \beta_{n-2 j+2 l+1}}\left\{\sum_{k=0}^{l}(-1)^{k}\left(\begin{array}{c}
j-k \\
l-1
\end{array}\right)\left(\begin{array}{l}
l \\
k
\end{array}\right)\right\} .
\end{aligned}
$$

It follows that the gauge variation vanishes after making use of (C.1) once more. In the second line of the above we have used the combinatoric factors to shift the upper and lower bounds of the summation over $j$. Then, in the third line we have shifted the dummy variable $j \mapsto j-k$.

\section{Integration by parts}

In this section we discuss integration by parts in conformal space. To demonstrate the technique we give a detailed analysis of how it works in the case of the $4 D$ CHS action (4.13) in a general curved space. However, before we begin the analysis let us give some general remarks.

To integrate by parts in $D$ dimensions, we must impose the gauge ${ }^{16}(2.25)$. As discussed earlier, the conformal covariant derivative then takes the form

$$
\mathfrak{b}_{a}=0 \quad \Longrightarrow \quad \nabla_{a}=\mathcal{D}_{a}+\frac{1}{2} P_{a}^{b} K_{b} .
$$

Consider some vector $V^{a}$, we obtain the following identity regarding total conformal derivatives

$$
\int \mathrm{d}^{D} x e \nabla_{a} V^{a}=\frac{1}{2} \int \mathrm{d}^{D} x e P_{a b} K^{a} V^{b} .
$$

In the usual way we have integrated out the total derivative arising from the torsion-free Lorentz covariant derivative. One then uses the conformal algebra and the conformal properties of the physical fields which comprise $V_{a}$ to eliminate the generator $K_{a}$.

Consider an integral of the form

$$
I=\int \mathrm{d}^{D} \text { xe } \mathcal{L}, \quad \mathbb{D} \mathcal{L}=D \mathcal{L}, \quad K_{a} \mathcal{L}=0 .
$$

This means that $I$ is invariant under the gauge group $\mathcal{G}$ and since $\mathcal{L}$ is primary all dependence on $\mathfrak{b}_{a}$ drops out. Let us further suppose that $\mathcal{L}$ takes the form

$$
\mathcal{L}=g^{J} \mathcal{A} h_{J}
$$

\footnotetext{
${ }^{16}$ In fact, since most Lagrangians we consider are primary, all dependence on $\mathfrak{b}_{a}$ drops out and we needn't choose the gauge $\mathfrak{b}_{a}=0$. However, the two are equivalent because in both cases the K-symmetry is exhausted.
} 
where $g_{J}$ and $h_{J}$ are primary fields with abstract index structure and $\mathcal{A}$ is a linear differential operator such that $\mathcal{A} h_{J} \equiv \mathcal{A}_{J}^{I} h_{I}$ is also primary. We define the transpose of the operator $\mathcal{A}$ by

$$
\int \mathrm{d}^{D} x e g^{J} \mathcal{A} h_{J}=\int \mathrm{d}^{D} x e h^{J} \mathcal{A}^{T} g_{J}+\int \mathrm{d}^{D} x e \Omega
$$

where $\Omega$ is a total conformal derivative and may be written as $\Omega=\nabla_{a} V^{a}$ for some vector $V^{a}$ with Weyl weight $D-1$. The first term on the right hand side of (D.5) is the result of integrating $I$ by parts in the usual way.

In general we cannot conclude that the second term on the right hand side of (D.5) vanishes. However, under the condition that $\mathcal{A}^{T} g_{J}$ is primary then $\Omega$ must also be primary. It follows that

$$
0=K_{a} \Omega=\left[K_{a}, \nabla_{b}\right] V^{b}+\nabla^{b} K_{a} V_{b}=\left(2 \eta_{a b} \mathbb{D}+2 M_{a b}\right) V^{b}+\nabla^{b} K_{a} V_{b}=\nabla^{b} K_{a} V_{b} .
$$

It is clear that the condition $\nabla^{b} K_{a} V_{b}=0$ is satisfied if $V_{a}$ is primary. What is not so clear is that any solution $V_{a}$ to this equation is necessarily primary. However, for all cases known to us this is true. Application of the rule (D.2) then allows us to conclude that the second term on right side of (D.5) vanishes up to a total derivative,

$$
\int \mathrm{d}^{D} x e \Omega=\int \mathrm{d}^{D} x e\left(\mathcal{D}^{a} V_{a}+\frac{1}{2} P^{a b} K_{a} V_{b}\right) \approx 0 .
$$

Therefore, we arrive at the following rule for integration by parts:

$$
\int \mathrm{d}^{D} x e g^{J} \mathcal{A} h_{J}=\int \mathrm{d}^{D} x e h^{J} \mathcal{A}^{T} g_{J}
$$

if $K_{a} g_{I}=K_{a} h_{I}=K_{a}\left(\mathcal{A} h_{I}\right)=K_{a}\left(\mathcal{A}^{T} g_{I}\right)=0$. We remark that most of the Lagrangians proposed in the main body of this paper are of the form (D.4).

As an example, presented below are the steps one must take in order to integrate the $4 D$ CHS action by parts in a general curved space. For convenience we do not include the complex conjugated part of the action.

$$
\begin{aligned}
& S_{\mathrm{CHS}}^{(m, n)}=\mathrm{i}^{m+n} \int \mathrm{d}^{4} x e \check{\mathfrak{C}}^{\alpha(m+n)} \hat{\mathfrak{C}}_{\alpha(m+n)} \\
& =\mathrm{i}^{m+n} \int \mathrm{d}^{4} x e(-1)^{n} \nabla_{\alpha_{1} \dot{\alpha}_{1}} \ldots \nabla_{\alpha_{m} \dot{\alpha}_{m}} \bar{\phi}_{\alpha_{m+1} \ldots \alpha_{m+n}} \dot{\alpha}_{1} \ldots \dot{\alpha}_{m} \hat{\mathfrak{C}}^{\alpha(m+n)} \\
& =\mathrm{i}^{m+n} \int \mathrm{d}^{4} x e(-1)^{n}\left\{(-1)^{m} \bar{\phi}_{\alpha_{m+1} \ldots \alpha_{m+n}} \dot{\alpha}_{1} \ldots \dot{\alpha}_{m} \nabla_{\alpha_{1} \dot{\alpha}_{1}} \ldots \nabla_{\alpha_{m} \dot{\alpha}_{m}} \hat{\mathfrak{C}}^{\alpha(m+n)}\right. \\
& \left.-\sum_{j=1}^{m}(-1)^{j} \nabla_{\alpha_{1} \dot{\alpha}_{1}}\left[\nabla_{\alpha_{j+1} \dot{\alpha}_{j+1}} \cdots \nabla_{\alpha_{m} \dot{\alpha}_{m}} \bar{\phi}_{\alpha_{m+1} \ldots \alpha_{m+n}}^{\dot{\alpha}_{1} \ldots \dot{\alpha}_{m}} \nabla_{\alpha_{2} \dot{\alpha}_{2}} \cdots \nabla_{\alpha_{j} \dot{\alpha}_{j}} \hat{\mathfrak{e}}^{\alpha(m+n)}\right]\right\} \\
& =\mathrm{i}^{m+n} \int \mathrm{d}^{4} x e \bar{\phi}^{\alpha(n) \dot{\alpha}(m)} \hat{\mathfrak{B}}_{\alpha(n) \dot{\alpha}(m)}-(-1)^{n} \mathrm{i}^{m+n} \int \mathrm{d}^{4} x e \nabla_{\alpha_{1} \dot{\alpha}_{1}} V^{\alpha_{1} \dot{\alpha}_{1}}
\end{aligned}
$$

where

$$
V^{\alpha_{1} \dot{\alpha}_{1}}=\sum_{j=1}^{m}(-1)^{j} \nabla_{\alpha_{j+1} \dot{\alpha}_{j+1}} \cdots \nabla_{\alpha_{m} \dot{\alpha}_{m}} \bar{\phi}_{\alpha_{m+1} \ldots \alpha_{m+n}}{ }_{\alpha_{1} \ldots \dot{\alpha}_{m}} \nabla_{\alpha_{2} \dot{\alpha}_{2}} \cdots \nabla_{\alpha_{j} \dot{\alpha}_{j}} \hat{\mathfrak{e}}^{\alpha(m+n)}
$$


In accordance with the previous discussion, since the integrand of the first term in (D.9) is of the form $h^{J} \mathcal{A}^{T} g_{J}$ and all primary conditions listed below (D.8) are met, the total conformal derivative must vanish. However, to support our belief that $V_{a}$ appearing in (D.6) is primary, we show that this is indeed the case for the current example,

$$
\begin{aligned}
& K_{\gamma \dot{\gamma}} V^{\alpha_{1} \dot{\alpha}_{1}}=\sum_{j=1}^{m-1}(-1)^{j} K_{\gamma \dot{\gamma}} \nabla_{\alpha_{j+1} \dot{\alpha}_{j+1}} \cdots \nabla_{\alpha_{m} \dot{\alpha}_{m}} \bar{\phi}_{\alpha_{m+1} \ldots \alpha_{m+n}}{ }_{\alpha_{1} \ldots \dot{\alpha}_{m}} \nabla_{\alpha_{2} \dot{\alpha}_{2}} \cdots \nabla_{\alpha_{j} \dot{\alpha}_{j}} \hat{\mathfrak{e}}^{\hat{\alpha}(m+n)} \\
& +\sum_{j=2}^{m}(-1)^{j} \nabla_{\alpha_{j+1} \dot{\alpha}_{j+1}} \cdots \nabla_{\alpha_{m} \dot{\alpha}_{m}} \bar{\phi}_{\alpha_{m+1} \ldots \alpha_{m+n}}{ }_{\dot{\alpha}_{1} \ldots \dot{\alpha}_{m}} K_{\gamma \dot{\gamma}} \nabla_{\alpha_{2} \dot{\alpha}_{2}} \cdots \nabla_{\alpha_{j} \dot{\alpha}_{j}} \hat{\mathfrak{C}}^{\alpha(m+n)} \\
& =\sum_{j=2}^{m}(-1)^{j}\left(\left[K_{\gamma \dot{\gamma}}, \nabla_{\alpha_{j} \dot{\alpha}_{j}} \cdots \nabla_{\alpha_{m} \dot{\alpha}_{m}}\right] \bar{\phi}_{\alpha_{m+1} \ldots \alpha_{m+n}}^{\dot{\alpha}_{1} \ldots \dot{\alpha}_{m}} \nabla_{\alpha_{2} \dot{\alpha}_{2}} \cdots \nabla_{\alpha_{j-1} \dot{\alpha}_{j-1}} \hat{\mathfrak{C}}^{\alpha(m+n)}\right.
\end{aligned}
$$

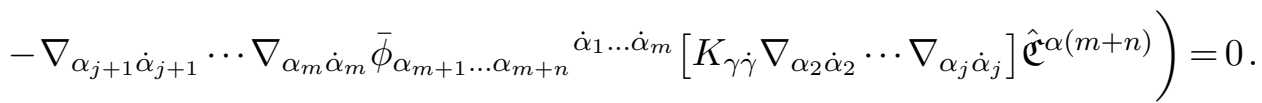

In the first line we have used the fact that the first term vanishes for $j=m$ whilst the second term vanishes for $j=1$. This allows us to translate the summation index of the first term in the second line. In going from the second to the third line, we have used the identity (4.8) twice, whereupon all terms in the round brackets cancel among themselves.

\section{E Conformal gravitino model}

As an application of the techniques developed earlier, we will discuss in detail the construction of a gauge-invariant model for the conformal gravitino in any $4 D$ Bach-flat spacetime. This model can be extracted from the action for $\mathcal{N}=1$ conformal supergravity [30,31] by linearising it around a Bach-flat background.

The conformal gravitino is described by a complex primary field $\phi_{\alpha(2) \dot{\alpha}}$ of dimension $+1 / 2$ and its conjugate, which are defined modulo gauge transformations of the type

$$
\delta_{\lambda} \phi_{\alpha(2) \dot{\alpha}}=\nabla_{\left(\alpha_{1} \dot{\alpha}\right.} \lambda_{\left.\alpha_{2}\right)},
$$

where the complex gauge parameter $\lambda_{\alpha}$ is primary of dimension $-1 / 2$.

Associated with the gravitino are the two field strengths

$$
\hat{\mathfrak{C}}_{\alpha(3)}=\nabla_{\left(\alpha_{1}\right.} \dot{\beta}_{\left.\alpha_{2} \alpha_{3}\right) \dot{\beta}}, \quad \check{\mathfrak{C}}_{\alpha(3)}=\nabla_{\left(\alpha_{1}\right.} \dot{\beta}_{1} \nabla_{\alpha_{2}} \dot{\beta}_{2} \bar{\phi}_{\left.\alpha_{3}\right) \dot{\beta}(2)},
$$

and their conjugates, which are primary fields of dimensions $+3 / 2$ and $+5 / 2$ respectively. Under the gauge transformation (E.1), their variations are given by

$$
\delta_{\lambda} \hat{\mathfrak{C}}_{\alpha(3)}=C_{\alpha(3) \delta} \lambda^{\delta}, \quad \delta_{\lambda} \check{\mathfrak{C}}_{\alpha(3)}=\frac{1}{2} C_{\alpha(3) \delta} \nabla^{\delta \dot{\delta}} \bar{\lambda}_{\dot{\delta}}-\bar{\lambda}_{\dot{\delta}} \nabla^{\delta \dot{\delta}} C_{\alpha(3) \delta} .
$$

In accordance with the results from section 4 , the action (4.13) with $m=2, n=1$, which we now denote by

$$
S_{\mathrm{CHS}}^{(3 / 2)}[\phi, \bar{\phi}]=-\mathrm{i} \int \mathrm{d}^{4} x \text { e } \hat{\mathfrak{C}}^{\alpha(3)} \check{\mathfrak{C}}_{\alpha(3)}+\text { c.c. },
$$


is invariant under the conformal gauge group $\mathcal{G}$, but not under (E.1). However, if (E.4) is supplemented by the non-minimal term that is linear in the Weyl tensor,

$$
\begin{gathered}
S_{\text {Linear }}^{(3 / 2)}=\mathrm{i} \int \mathrm{d}^{4} x \text { e } \phi^{\alpha(2) \dot{\alpha}} \breve{\mathfrak{J}}_{\alpha(2) \dot{\alpha}}+\text { c.c. } \\
\check{\mathfrak{J}}_{\alpha(2) \dot{\alpha}}=C_{\alpha(2)}{ }^{\beta(2)} \nabla_{\beta_{1}} \dot{\beta} \bar{\phi}_{\beta_{2} \dot{\alpha} \dot{\beta}}-\bar{\phi}_{\beta_{1} \dot{\alpha} \dot{\beta}} \nabla_{\beta_{2}}{ }^{\dot{\beta}} C_{\alpha(2)}{ }^{\beta(2)}
\end{gathered}
$$

where $\check{\mathfrak{J}}_{\alpha(2) \dot{\alpha}}$ is a dimension $+7 / 2$ primary field, then the resulting action

$$
S_{\text {Gravitino }}=S_{\text {CHS }}^{(3 / 2)}+S_{\text {Linear }}^{(3 / 2)}
$$

is invariant under (E.1) provided the background Bach tensor (4.42) vanishes,

$$
B_{\alpha(2) \dot{\alpha}(2)}=0 \text {. }
$$

We remark that the following primary deformation of the linearised Bach tensor,

$$
\check{\mathcal{B}}_{\alpha(2) \dot{\alpha}}=\check{\mathfrak{B}}_{\alpha(2) \dot{\alpha}}-\check{\mathfrak{J}}_{\alpha(2) \dot{\alpha}},
$$

which may be used to rewrite the action (E.7) as

$$
S_{\text {Gravitino }}=-\mathrm{i} \int \mathrm{d}^{4} x e \phi^{\alpha(2) \dot{\alpha}} \check{\mathcal{B}}_{\alpha(2) \dot{\alpha}}+\text { c.c. },
$$

is transverse and gauge invariant in any Bach-flat spacetime,

$$
B_{\alpha(2) \dot{\alpha}(2)}=0 \quad \Longrightarrow \quad \nabla^{\gamma \dot{\gamma}} \check{\mathcal{B}}_{\alpha \gamma \dot{\gamma}}=\delta_{\lambda} \check{\mathcal{B}}_{\alpha(2) \dot{\alpha}}=0 .
$$

To conclude, we present the degauged versions of the above results. In the gauge (2.25), the gauge transformations (E.1) are

$$
\delta_{\lambda} \phi_{\alpha(2) \dot{\alpha}}=\mathcal{D}_{\left(\alpha_{1} \dot{\alpha}\right.} \lambda_{\left.\alpha_{2}\right)} .
$$

Under (E.12), the degauged gravitino field strengths

$$
\hat{\mathfrak{C}}_{\alpha(3)}=\mathcal{D}_{\left(\alpha_{1}\right.} \dot{\beta} \phi_{\left.\alpha_{2} \alpha_{3}\right) \dot{\beta}}, \quad \check{\mathfrak{C}}_{\alpha(3)}=\mathcal{D}_{\left(\alpha_{1}\right.} \dot{\beta}_{1} \mathcal{D}_{\alpha_{2}} \dot{\beta}_{2} \bar{\phi}_{\left.\alpha_{3}\right) \dot{\beta}(2)}-\frac{1}{2} R_{\left(\alpha_{1} \alpha_{2}\right.} \dot{\beta}(2) \bar{\phi}_{\left.\alpha_{3}\right) \dot{\beta}(2)},
$$

transform as

$$
\delta_{\lambda} \hat{\mathfrak{C}}_{\alpha(3)}=C_{\alpha(3) \delta} \lambda^{\delta}, \quad \delta_{\lambda} \check{\mathfrak{C}}_{\alpha(3)}=\frac{1}{2} C_{\alpha(3) \delta} \mathcal{D}^{\delta \dot{\delta}} \bar{\lambda}_{\dot{\delta}}-\bar{\lambda}_{\dot{\delta}} \mathcal{D}^{\delta \dot{\delta}} C_{\alpha(3) \delta} .
$$

The degauged gravitino action (E.7) remains the same except with (E.2) replaced by (E.13) in $S_{\text {CHS }}^{(3 / 2)}$ as well as the replacement $\nabla_{\alpha \dot{\alpha}} \mapsto \mathcal{D}_{\alpha \dot{\alpha}}$ in $S_{\text {Linear }}^{(3 / 2)}$. Finally, $S_{\text {Gravitino is invariant }}$ under the gauge transformations (E.12) as long as the degauged Bach tensor,

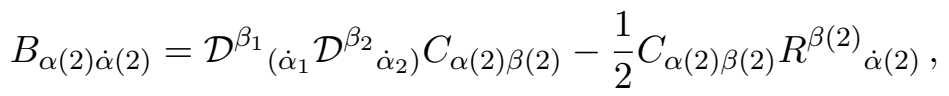

vanishes. 


\section{F Conformal graviton model}

In this appendix we construct a conformally invariant model for the graviton that is gauge invariant in any Bach-flat spacetime. This model may of course be obtained by linearising the action of $4 D$ conformal gravity around a Bach-flat background. However, in principle the method presented below can be applied to higher-spin models, albeit with considerable effort.

In accordance with section 4, the conformal graviton is described by a real primary field $h_{\alpha(2) \dot{\alpha}(2)}=\bar{h}_{\alpha(2) \dot{\alpha}(2)}$ with zero Weyl weight and is defined modulo the gauge transformations

$$
\delta_{\lambda} h_{\alpha(2) \dot{\alpha}(2)}=\nabla_{\left(\alpha _ { 1 } \left(\dot{\alpha}_{1}\right.\right.} \lambda_{\left.\left.\alpha_{2}\right) \dot{\alpha}_{2}\right)} .
$$

Associated with the graviton is the linearised Weyl tensor,

$$
\mathfrak{C}_{\alpha(4)}=\nabla_{\left(\alpha_{1}\right.}{ }^{\dot{\beta}_{1}} \nabla_{\alpha_{2}} \dot{\beta}_{2} h_{\left.\alpha_{3} \alpha_{4}\right) \dot{\beta}_{1} \dot{\beta}_{2}}
$$

which is a primary field of dimension 2. Under the gauge transformation (F.1), its variation is given by

$$
\delta_{\lambda} \mathfrak{C}_{\alpha(4)}=\frac{1}{2} C_{\alpha(4)} \nabla^{\beta \dot{\beta}} \lambda_{\beta \dot{\beta}}-\lambda_{\beta \dot{\beta}} \nabla^{\beta \dot{\beta}} C_{\alpha(4)}-2 C^{\beta}{ }_{\left(\alpha_{1} \alpha_{2} \alpha_{3}\right.} \nabla_{\left.\alpha_{4}\right)}{ }^{\dot{\beta}} \lambda_{\beta \dot{\beta}}
$$

The action of linearised conformal gravity is given by (4.13), with $m=n=2$, which we now denote by $S_{\mathrm{CHS}}^{(2)}$,

$$
S_{\mathrm{CHS}}^{(2)}=\int \mathrm{d}^{4} x e \mathfrak{C}^{\alpha(4)} \mathfrak{C}_{\alpha(4)}+\text { c.c. }
$$

In general (F.4) is invariant under the gauge group $\mathcal{G}$, but only in conformally flat spacetimes is it invariant under (F.1). Indeed, upon integrating by parts, we find that under (F.1) the action (F.4) varies as

$$
\begin{aligned}
\delta_{\lambda} S_{\mathrm{CHS}}^{(2)}=\int \mathrm{d}^{4} x e \lambda^{\alpha \dot{\alpha}}\left\{4 \mathfrak{C}^{\beta(4)} \nabla_{\beta \dot{\alpha}} C_{\beta(3) \alpha}\right. & +4 C_{\beta(3) \alpha} \nabla_{\beta \dot{\alpha}} \mathfrak{C}^{\beta(4)} \\
& \left.-C_{\beta(4)} \nabla_{\alpha \dot{\alpha}} \mathfrak{C}^{\beta(4)}-3 \mathfrak{C}^{\beta(4)} \nabla_{\alpha \dot{\alpha}} C_{\beta(4)}\right\}+ \text { c.c. }
\end{aligned}
$$

In the spirit of the previous appendix, to extend the gauge invariance of this model we seek a weight +4 primary deformation of the linearised Bach tensor, denoted by $\mathfrak{J}_{\alpha(2) \dot{\alpha}(2)}$,

$$
K_{\beta \dot{\beta}} \mathfrak{J}_{\alpha(2) \dot{\alpha}(2)}=0, \quad \mathbb{D}_{\mathfrak{J}_{\alpha(2) \dot{\alpha}(2)}}=4 \mathfrak{J}_{\alpha(2) \dot{\alpha}(2)} .
$$

Restricting our attention to the construction of tensors with the properties of $\mathfrak{J}_{\alpha(2) \dot{\alpha}(2)}$ greatly lightens the workload. In fact, beginning with the most general weight +4 tensor with this index structure, the condition of being primary is so strong that one may show that there are only three (up to complex conjugation) such inequivalent tensors that are 
linear in the Weyl tensor. They are given by

$$
\begin{aligned}
& \mathfrak{J}_{\alpha(2) \dot{\alpha}(2)}^{1}=B_{\alpha_{1}}{ }^{\gamma \dot{\gamma}} \dot{\alpha}_{1} h_{\alpha_{2} \gamma \dot{\gamma} \dot{\alpha}_{2}}, \\
& \mathfrak{J}_{\alpha(2) \dot{\alpha}(2)}^{2}=-2 C_{\alpha(2)}{ }^{\beta(2)} \nabla_{\dot{\alpha}_{1}}{ }^{\gamma} \nabla_{\beta_{1}}{ }^{\dot{\gamma}} h_{\gamma \beta_{2} \dot{\alpha}_{2} \dot{\gamma}}-\nabla_{\dot{\alpha}_{1}}{ }^{\gamma} C_{\alpha(2)}{ }^{\beta(2)} \nabla_{\gamma}{ }^{\dot{\gamma}} h_{\beta(2) \dot{\alpha}_{2} \dot{\gamma}} \\
& +2 \nabla_{\dot{\alpha}_{1}}^{\gamma} C_{\alpha(2)}^{\beta(2)} \nabla_{\beta_{1}}{ }^{\dot{\gamma}} h_{\beta_{2} \gamma \dot{\alpha} 2} \dot{\gamma}-\nabla_{\alpha_{1} \dot{\alpha}_{1}} C_{\alpha_{2}}{ }^{\beta(3)} \nabla_{\beta_{1}}{ }^{\dot{\gamma}} h_{\beta_{2} \beta_{3} \dot{\alpha}_{2} \dot{\gamma}} \\
& -\nabla_{\alpha_{1}} \dot{\gamma} C_{\alpha_{2}}{ }^{\beta(3)} \nabla_{\beta_{1} \dot{\alpha}_{1}} h_{\beta_{2} \beta_{3} \dot{\alpha}_{2} \dot{\gamma}}+3 \nabla_{\beta_{1}}{ }^{\dot{\gamma}} C_{\alpha(2)}{ }^{\beta(2)} \nabla_{\dot{\gamma}}{ }^{\gamma} h_{\gamma \beta_{2} \dot{\alpha}(2)} \\
& +\frac{1}{2} \nabla^{\gamma \dot{\gamma}} C_{\alpha(2)}^{\beta(2)} \nabla_{\gamma \dot{\gamma}} h_{\beta(2) \dot{\alpha}(2)}+h_{\beta(2) \dot{\alpha}(2)} \square_{c} C_{\alpha(2)}{ }^{\beta(2)} \text {, } \\
& \mathfrak{J}_{\alpha(2) \dot{\alpha}(2)}^{3}=-C_{\alpha_{1}}{ }^{\beta(3)} \nabla_{\beta_{1} \dot{\alpha}_{1}} \nabla_{\beta_{2}}{ }^{\dot{\gamma}} h_{\beta_{3} \alpha_{2} \dot{\alpha}_{2} \dot{\gamma}}+C_{\alpha(2)}{ }^{\beta(2)} \square_{c} h_{\beta(2) \dot{\alpha}(2)} \\
& -2 \nabla_{\beta_{1} \dot{\alpha}_{1}} C_{\alpha_{1}}{ }^{\beta(3)} \nabla_{\alpha_{2}}{ }^{\dot{\gamma}} h_{\beta_{2} \beta_{3} \dot{\alpha}_{2} \dot{\gamma}}-\nabla_{\beta_{1}}{ }^{\dot{\gamma}} C_{\alpha(2)}{ }^{\beta(2)} \nabla_{\dot{\gamma}}^{\gamma} h_{\beta_{2} \gamma \dot{\alpha}(2)} \\
& -\frac{1}{2} \nabla^{\gamma \dot{\gamma}} C_{\alpha(2)}{ }^{\beta(2)} \nabla_{\gamma \dot{\gamma}} h_{\beta(2) \dot{\alpha}(2)}-h_{\gamma \beta_{1} \dot{\gamma} \dot{\alpha}_{1}} \nabla_{\dot{\alpha}_{2}}{ }^{\gamma} \nabla_{\beta_{2}}{ }^{\dot{\gamma}} C_{\alpha(2)}{ }^{\beta(2)} \\
& +h_{\beta(2) \dot{\alpha}(2)} \square_{c} C_{\alpha(2)}^{\beta(2)} \text {. }
\end{aligned}
$$

In the above and for the remainder of this appendix we employ the convention whereby all free dotted and undotted indices appearing in any tensor are assumed to be independently symmetrised over, e.g., $\nabla_{\alpha_{1} \dot{\alpha}_{1}} C_{\alpha_{2}}{ }^{\beta(3)} \nabla_{\beta_{1}}{ }^{\dot{\gamma}} h_{\beta_{2} \beta_{3} \dot{\alpha}_{2} \dot{\gamma}}=$ $\nabla_{\left(\alpha_{1}\left(\dot{\alpha}_{1}\right.\right.} C_{\left.\alpha_{2}\right)}{ }^{\beta(3)} \nabla_{\mid \beta_{1}} \dot{\gamma} h_{\left.\beta_{2} \beta_{3} \mid \dot{\alpha}_{2}\right) \dot{\gamma}}$.

In addition to the primary fields (F.7a), (F.7b) and (F.7c), there are precisely three (up to complex conjugation) inequivalent structures that are quadratic in the Weyl tensor and which satisfy the properties (F.6). They are given by

$$
\begin{aligned}
& \mathfrak{J}_{\alpha(2) \dot{\alpha}(2)}^{4}=C_{\alpha(2)}{ }^{\gamma(2)} C_{\gamma(2)}{ }^{\beta(2)} h_{\beta(2) \dot{\alpha}(2)}, \\
& \mathfrak{J}_{\alpha(2) \dot{\alpha}(2)}^{5}=C_{\alpha_{1} \gamma(2)}{ }^{\beta_{1}} C_{\alpha_{2}}{ }^{\beta_{2} \gamma(2)} h_{\beta(2) \dot{\alpha}(2)}, \\
& \mathfrak{J}_{\alpha(2) \dot{\alpha}(2)}^{6}=C_{\alpha(2)}{ }^{\beta(2)} \bar{C}_{\dot{\alpha}(2)}{ }^{\dot{\beta}(2)} h_{\beta(2) \dot{\beta}(2)} .
\end{aligned}
$$

The tensors (F.7) span all primary structures of the type $\mathfrak{J}_{\alpha(2) \dot{\alpha}(2)}$ and in particular any linear combination will also satisfy (F.6). Furthermore, if we express them in the form

$$
\mathfrak{J}_{\alpha(2) \dot{\alpha}(2)}^{i}=\mathcal{A}_{i} h_{\alpha(2) \dot{\alpha}(2)}
$$

where $\mathcal{A}_{i}$ is a linear differential operator then, with the exception of $\mathcal{A}_{2}$, it may be shown that each operator is symmetric in the sense $\mathcal{A}_{i}=\mathcal{A}_{i}^{T}$ (see appendix $\mathrm{D}$ for the definition of $\mathcal{A}_{i}^{T}$ ). This property reduces the amount of work required to compute the gauge variation of each of the functionals associated with $\mathfrak{J}_{\alpha(2) \dot{\alpha}(2)}^{i}$.

Any operator $\mathcal{A}$ may be decomposed into symmetric and antisymmetric parts, $\mathcal{A}=$ $\mathcal{A}_{\mathrm{S}}+\mathcal{A}_{\mathrm{A}}$ with $\mathcal{A}_{\mathrm{S}}=\frac{1}{2}\left(\mathcal{A}+\mathcal{A}^{T}\right)$ and $\mathcal{A}_{\mathrm{A}}=\frac{1}{2}\left(\mathcal{A}-\mathcal{A}^{T}\right)$. It follows that the antisymmetric part of $\mathcal{A}$ vanishes identically in any integral of the form

$$
\int \mathrm{d}^{4} x e h^{J} \mathcal{A} h_{J}=\int \mathrm{d}^{4} x e h^{J} \mathcal{A}_{\mathrm{S}} h_{J}
$$


Using (F.9) it is possible to show that at the level of actions, the following correspondence between $\mathfrak{J}^{2}$ and the remaining primary structures holds

$$
\int \mathrm{d}^{4} x e h^{\alpha(2) \dot{\alpha}(2)} \mathfrak{J}_{\alpha(2) \dot{\alpha}(2)}^{2}=\int \mathrm{d}^{4} x e h^{\alpha(2) \dot{\alpha}(2)}\left(2 \mathcal{A}_{1}-\mathcal{A}_{3}+2 \mathcal{A}_{4}+\mathcal{A}_{5}+\mathcal{A}_{6}\right) h_{\alpha(2) \dot{\alpha}(2)} .
$$

Additionally, the structure $\mathfrak{J}^{1}$ vanishes in any Bach-flat spacetime and will be of no use. Therefore, it suffices to consider only one of the primary structures that is linear in the Weyl tensor, say $\mathfrak{J}^{3}$, and its associated functional

$$
S_{\text {Linear }}^{(2)}=\int \mathrm{d}^{4} x e h^{\alpha(2) \dot{\alpha}(2)} \mathfrak{J}_{\alpha(2) \dot{\alpha}(2)}^{3}+\text { c.c. }
$$

One can then show that under the gauge transformation (F.1) and upon integrating by parts, the action (F.11) transforms as

$$
\begin{aligned}
\delta_{\lambda} S_{\text {Linear }}^{(2)}= & \frac{1}{2} \delta_{\lambda} S_{\mathrm{CHS}}^{(2)}+\left(2 \int \mathrm { d } ^ { 4 } x e \lambda ^ { \alpha \dot { \alpha } } \left\{\nabla_{\delta}^{\dot{\delta}}\left[C_{\gamma(2)}^{\beta(2)} C_{\alpha}{ }^{\gamma(2) \delta} h_{\beta(2) \dot{\alpha} \dot{\delta}}\right]\right.\right. \\
& \left.+\nabla_{\beta_{1}}^{\dot{\delta}}\left[C^{\beta(2)} \gamma(2) C_{\alpha}^{\gamma(2) \delta} h_{\beta_{2} \delta \dot{\alpha} \dot{\delta}}\right]-\nabla_{\beta_{1} \dot{\beta}_{1}}\left[C_{\alpha}{ }^{\beta(3)} \bar{C}_{\dot{\alpha}}^{\dot{\beta}(3)} h_{\beta(2) \dot{\beta}(2)}\right]\right\} \\
& \left.-\int \mathrm{d}^{4} x e \lambda^{\alpha \dot{\alpha}}\left\{\nabla^{\beta_{1} \dot{\beta}_{1}} B_{\alpha}{ }^{\beta_{2} \dot{\beta}_{2}} \dot{\alpha} h_{\beta(2) \dot{\beta}(2)}+B_{\alpha}{ }^{\beta_{1} \dot{\beta}(2)} \nabla_{\dot{\alpha}}{ }^{\beta_{2}} h_{\beta(2) \dot{\beta}(2)}\right\}+\text { c.c. }\right) .
\end{aligned}
$$

To annihilate the terms quadratic in the Weyl tensor, we define the functional

$$
S_{\text {Quadratic }}^{(2)}=\int \mathrm{d}^{4} x e h^{\alpha(2) \dot{\alpha}(2)}\left\{\mathfrak{J}_{\alpha(2) \dot{\alpha}(2)}^{4}+\mathfrak{J}_{\alpha(2) \dot{\alpha}(2)}^{5}+\mathfrak{J}_{\alpha(2) \dot{\alpha}(2)}^{6}\right\}+\text { c.c. }
$$

It follows that the action

$$
\begin{aligned}
& S_{\text {Graviton }}=S_{\text {CHS }}^{(2)}-2 S_{\text {Linear }}^{(2)}+2 S_{\text {Quadratic }}^{(2)} \\
& =\int \mathrm{d}^{4} x e h^{\alpha(2) \dot{\alpha}(2)}\left\{\mathfrak{B}_{\alpha(2) \dot{\alpha}(2)}+2 C_{\alpha_{1}}{ }^{\beta(3)} \nabla_{\beta_{1} \dot{\alpha}_{1}} \nabla_{\beta_{2}}{ }^{\dot{\gamma}} h_{\beta_{3} \alpha_{2} \dot{\alpha}_{2} \dot{\gamma}}-2 C_{\alpha(2)}{ }^{\beta(2)} \square_{c} h_{\beta(2) \dot{\alpha}(2)}\right. \\
& \quad+4 \nabla_{\beta_{1} \dot{\alpha}_{1}} C_{\alpha_{1}}{ }^{\beta(3)} \nabla_{\alpha_{2}}{ }^{\dot{\gamma}} h_{\beta_{2} \beta_{3} \dot{\alpha} 2 \dot{\gamma}}+2 \nabla_{\beta_{1}}{ }^{\dot{\gamma}} C_{\alpha(2)}{ }^{\beta(2)} \nabla_{\dot{\gamma}}{ }^{\gamma} h_{\beta_{2} \gamma \dot{\alpha}(2)}-2 h_{\beta(2) \dot{\alpha}(2)} \square_{c} C_{\alpha(2)}{ }^{\beta(2)} \\
& \quad+\nabla^{\gamma \dot{\gamma}} C_{\alpha(2)}{ }^{\beta(2)} \nabla_{\gamma \dot{\gamma}} h_{\beta(2) \dot{\alpha}(2)}+2 h_{\gamma \beta_{1} \dot{\gamma} \dot{\alpha}_{1}} \nabla_{\dot{\alpha}_{2}}{ }^{\gamma} \nabla_{\beta_{2}}{ }^{\dot{\gamma}} C_{\alpha(2)}{ }^{\beta(2)}+2 C_{\alpha(2)}{ }^{\gamma(2)} C_{\gamma(2)}{ }^{\beta(2)} h_{\beta(2) \dot{\alpha}(2)} \\
& \left.\quad+2 C_{\alpha_{1} \gamma(2)}{ }^{\beta_{1}} C_{\alpha_{2}}{ }^{\beta_{2} \gamma(2)} h_{\beta(2) \dot{\alpha}(2)}+2 C_{\alpha(2)}{ }^{\beta(2)} \bar{C}_{\dot{\alpha}(2)}{ }^{\dot{\beta}(2)} h_{\beta(2) \dot{\beta}(2)}\right\}+ \text { c.c. },
\end{aligned}
$$

whose variation under (F.1) is given by

$$
\delta_{\lambda} S_{\text {Graviton }}=2 \int \mathrm{d}^{4} x e \lambda^{\alpha \dot{\alpha}}\left\{\nabla^{\beta_{1} \dot{\beta}_{1}} B_{\alpha}^{\beta_{2} \dot{\beta}_{2}} h_{\beta(2) \dot{\beta}(2)}+B_{\alpha}^{\beta_{1} \dot{\beta}(2)} \nabla_{\dot{\alpha}}^{\beta_{2}} h_{\beta(2) \dot{\beta}(2)}\right\}+\text { c.c. }
$$

is the unique model describing the graviton that is both conformally invariant in a general curved spacetime and gauge invariant in any Bach-flat spacetime,

$$
B_{\alpha(2) \dot{\alpha}(2)}=0 \quad \Longrightarrow \quad \delta_{\lambda} S_{\text {Graviton }}=0 .
$$


This model was analysed in [29] using a similar methodology, the main differences being that their analysis was performed in the gauge $\mathfrak{b}_{a}=0$ and the graviton field was not traceless. In accordance with (1.1), this means that their model contains an extra algebraic gauge symmetry which may be used to gauge away the trace. The authors found two inequivalent primary Lagrangians that were linear in the Weyl tensor and used both in the construction of their gauge invariant action. Upon eliminating the trace of the graviton field, one of these structures vanishes and the other must be proportional to (F.11) modulo terms involving the Bach tensor and the square of the Weyl tensor.

Finally, we remark that the following primary deformation of the linearised Bach tensor,

$$
\mathcal{B}_{\alpha(2) \dot{\alpha}(2)}=\mathfrak{B}_{\alpha(2) \dot{\alpha}(2)}-2 \mathfrak{J}_{\alpha(2) \dot{\alpha}(2)}^{3}+2 \mathfrak{J}_{\alpha(2) \dot{\alpha}(2)}^{4}+2 \mathfrak{J}_{\alpha(2) \dot{\alpha}(2)}^{5}+2 \mathfrak{J}_{\alpha(2) \dot{\alpha}(2)}^{6},
$$

which may be used to rewrite the action (F.14) as

$$
S_{\text {Graviton }}=\int \mathrm{d}^{4} x e h^{\alpha(2) \dot{\alpha}(2)} \mathcal{B}_{\alpha(2) \dot{\alpha}(2)},
$$

is transverse and gauge invariant in any Bach-flat spacetime,

$$
B_{\alpha(2) \dot{\alpha}(2)}=0 \quad \Longrightarrow \quad \nabla^{\gamma \dot{\gamma}} \mathcal{B}_{\alpha \gamma \dot{\gamma} \dot{\alpha}}=\delta_{\lambda} \mathcal{B}_{\alpha(2) \dot{\alpha}(2)}=0 .
$$

Open Access. This article is distributed under the terms of the Creative Commons Attribution License (CC-BY 4.0), which permits any use, distribution and reproduction in any medium, provided the original author(s) and source are credited.

\section{References}

[1] E.S. Fradkin and A.A. Tseytlin, Conformal supergravity, Phys. Rept. 119 (1985) 233 [INSPIRE].

[2] E.S. Fradkin and V. Ya. Linetsky, A superconformal theory of massless higher spin fields in $D=(2+1)$, Mod. Phys. Lett. A 4 (1989) 731 [InSPIRE].

[3] C.N. Pope and P.K. Townsend, Conformal higher spin in $(2+1)$ dimensions, Phys. Lett. B 225 (1989) 245 [inSPIRE].

[4] E.S. Fradkin and V. Ya. Linetsky, Cubic interaction in conformal theory of integer higher-spin fields in four dimensional space-time, Phys. Lett. B 231 (1989) 97 [INSPIRE].

[5] E.S. Fradkin and V. Ya. Linetsky, Superconformal higher spin theory in the cubic approximation, Nucl. Phys. B 350 (1991) 274 [INSPIRE].

[6] A.A. Tseytlin, On limits of superstring in AdS(5) x $S^{* * 5}$, Theor. Math. Phys. 133 (2002) 1376 [Teor. Mat. Fiz. 133 (2002) 69] [hep-th/0201112] [INSPIRE].

[7] A.Y. Segal, Conformal higher spin theory, Nucl. Phys. B 664 (2003) 59 [hep-th/0207212] [INSPIRE].

[8] R. Marnelius, Lagrangian conformal higher spin theory, arXiv:0805.4686 [INSPIRE].

[9] R.R. Metsaev, Gauge invariant two-point vertices of shadow fields, AdS/CFT and conformal fields, Phys. Rev. D 81 (2010) 106002 [arXiv: 0907.4678] [InSPIRE]. 
[10] M.A. Vasiliev, Bosonic conformal higher-spin fields of any symmetry, Nucl. Phys. B 829 (2010) 176 [arXiv:0909.5226] [inSPIRE].

[11] M. Beccaria, X. Bekaert and A.A. Tseytlin, Partition function of free conformal higher spin theory, JHEP 08 (2014) 113 [arXiv: 1406.3542] [INSPIRE].

[12] M. Beccaria and A.A. Tseytlin, On higher spin partition functions, J. Phys. A 48 (2015) 275401 [arXiv: 1503.08143] [INSPIRE].

[13] M. Beccaria, S. Nakach and A.A. Tseytlin, On triviality of S-matrix in conformal higher spin theory, JHEP 09 (2016) 034 [arXiv: 1607.06379] [INSPIRE].

[14] T. Adamo, S. Nakach and A.A. Tseytlin, Scattering of conformal higher spin fields, JHEP 07 (2018) 016 [arXiv: 1805.00394] [INSPIRE].

[15] M. Beccaria and A.A. Tseytlin, Superconformal index of higher derivative $\mathcal{N}=1$ multiplets in four dimensions, JHEP 10 (2018) 087 [arXiv:1807.05911] [INSPIRE].

[16] P.S. Howe, K.S. Stelle and P.K. Townsend, Supercurrents, Nucl. Phys. B 192 (1981) 332 [INSPIRE].

[17] S.M. Kuzenko, R. Manvelyan and S. Theisen, Off-shell superconformal higher spin multiplets in four dimensions, JHEP 07 (2017) 034 [arXiv:1701.00682] [INSPIRE].

[18] S.M. Kuzenko, Higher spin super-Cotton tensors and generalisations of the linear-chiral duality in three dimensions, Phys. Lett. B 763 (2016) 308 [arXiv: 1606.08624] [INSPIRE].

[19] S.M. Kuzenko and M. Tsulaia, Off-shell massive $N=1$ supermultiplets in three dimensions, Nucl. Phys. B 914 (2017) 160 [arXiv:1609.06910] [INSPIRE].

[20] S.M. Kuzenko and M. Ponds, Topologically massive higher spin gauge theories, JHEP 10 (2018) 160 [arXiv: 1806.06643] [INSPIRE].

[21] S.M. Kuzenko and D.X. Ogburn, Off-shell higher spin $N=2$ supermultiplets in three dimensions, Phys. Rev. D 94 (2016) 106010 [arXiv:1603.04668] [INSPIRE].

[22] J. Hutomo, S.M. Kuzenko and D. Ogburn, $\mathcal{N}=2$ supersymmetric higher spin gauge theories and current multiplets in three dimensions, Phys. Rev. D 98 (2018) 125004 [arXiv: 1807.09098] [INSPIRE].

[23] A. Yu. Segal, Point particle in general background fields vsersus gauge theories of traceless symmetric tensors, Int. J. Mod. Phys. A 18 (2003) 4999 [hep-th/0110056] [INSPIRE].

[24] X. Bekaert, E. Joung and J. Mourad, Effective action in a higher-spin background, JHEP 02 (2011) 048 [arXiv: 1012.2103] [INSPIRE].

[25] R. Bonezzi, Induced action for conformal higher spins from worldline path integrals, Universe 3 (2017) 64 [arXiv:1709.00850] [INSPIRE].

[26] T. Nutma and M. Taronna, On conformal higher spin wave operators, JHEP 06 (2014) 066 [arXiv: 1404.7452] [INSPIRE].

[27] M. Grigoriev and A.A. Tseytlin, On conformal higher spins in curved background, J. Phys. A 50 (2017) 125401 [arXiv: 1609.09381] [INSPIRE].

[28] M. Beccaria and A.A. Tseytlin, On induced action for conformal higher spins in curved background, Nucl. Phys. B 919 (2017) 359 [arXiv:1702.00222] [INSPIRE].

[29] R. Manvelyan and G. Poghosyan, Geometrical structure of Weyl invariants for spin three gauge field in general gravitational background in d =4, Nucl. Phys. B 937 (2018) 1 [arXiv: 1804.10779] [INSPIRE]. 
[30] M. Kaku, P.K. Townsend and P. van Nieuwenhuizen, Gauge theory of the conformal and superconformal group, Phys. Lett. 69B (1977) 304 [INSPIRE].

[31] M. Kaku, P.K. Townsend and P. van Nieuwenhuizen, Properties of conformal supergravity, Phys. Rev. D 17 (1978) 3179 [INSPIRE].

[32] I.L. Buchbinder and S.M. Kuzenko, Quantization of the classically equivalent theories in the superspace of simple supergravity and quantum equivalence, Nucl. Phys. B 308 (1988) 162 [INSPIRE].

[33] I.L. Buchbinder and S.M. Kuzenko, Ideas and Methods of Supersymmetry and Supergravity or a Walk Through Superspace, IOP, Bristol, U.K. (1995), (Revised Edition: (1998)).

[34] S. Ferrara and B. Zumino, Structure of linearized supergravity and conformal supergravity, Nucl. Phys. B 134 (1978) 301 [inSPIRE].

[35] S. Deser, R. Jackiw and S. Templeton, Three-dimensional massive gauge theories, Phys. Rev. Lett. 48 (1982) 975 [INSPIRE].

[36] S. Deser, R. Jackiw and S. Templeton, Topologically massive gauge theories, Annals Phys. 140 (1982) 372 [Erratum ibid. 185 (1988) 406] [INSPIRE].

[37] P. van Nieuwenhuizen, $D=3$ conformal supergravity and Chern-Simons terms, Phys. Rev. D 32 (1985) 872 [INSPIRE].

[38] D. Butter, S.M. Kuzenko, J. Novak and G. Tartaglino-Mazzucchelli, Conformal supergravity in three dimensions: New off-shell formulation, JHEP 09 (2013) 072 [arXiv:1305.3132] [INSPIRE].

[39] D. Butter, S.M. Kuzenko, J. Novak and G. Tartaglino-Mazzucchelli, Conformal supergravity in three dimensions: Off-shell actions, JHEP 10 (2013) 073 [arXiv: 1306.1205] [INSPIRE].

[40] S.M. Kuzenko, J. Novak and G. Tartaglino-Mazzucchelli, $N=6$ superconformal gravity in three dimensions from superspace, JHEP 01 (2014) 121 [arXiv: 1308.5552] [INSPIRE].

[41] D. Butter, $N=1$ conformal superspace in four dimensions, Annals Phys. 325 (2010) 1026 [arXiv: 0906.4399] [INSPIRE].

[42] D. Butter, $N=2$ conformal superspace in four dimensions, JHEP 10 (2011) 030 [arXiv:1103.5914] [INSPIRE].

[43] D. Butter, S.M. Kuzenko, J. Novak and G. Tartaglino-Mazzucchelli, Conformal supergravity in five dimensions: New approach and applications, JHEP 02 (2015) 111 [arXiv: 1410.8682] [INSPIRE].

[44] D. Butter, S.M. Kuzenko, J. Novak and S. Theisen, Invariants for minimal conformal supergravity in six dimensions, JHEP 12 (2016) 072 [arXiv: 1606.02921] [INSPIRE].

[45] C. Fronsdal, Massless fields with integer spin, Phys. Rev. D 18 (1978) 3624 [INSPIRE].

[46] C. Fronsdal, Singletons and massless, integral-spin fields on de Sitter space, Phys. Rev. D 20 (1979) 848 [INSPIRE].

[47] M.A. Vasiliev, "Gauge" form of description of massless fields with arbitrary spin, Sov. J. Nucl. Phys. 32 (1980) 439 [Yad. Fiz. 32 (1980) 855] [INSPIRE].

[48] M.A. Vasiliev, Free massless fields of arbitrary spin in the de Sitter space and initial data for a higher spin superalgebra, Fortsch. Phys. 35 (1987) 741 [InSPIRE].

[49] E. Bergshoeff, M. de Roo and B. de Wit, Extended conformal supergravity, Nucl. Phys. B 182 (1981) 173 [inSPIRE].

[50] D.Z. Freedman and A. Van Proeyen, Supergravity, Cambridge University Press, (2012). 
[51] J.H. Horne and E. Witten, Conformal gravity in three dimensions as a gauge theory, Phys. Rev. Lett. 62 (1989) 501 [INSPIRE].

[52] J. Wess and J. Bagger, Supersymmetry and Supergravity, Princeton University Press, (1992).

[53] E.I. Buchbinder, S.M. Kuzenko, J. La Fontaine and M. Ponds, Spin projection operators and higher-spin Cotton tensors in three dimensions, Phys. Lett. B 790 (2019) 389 [arXiv: 1812.05331] [INSPIRE].

[54] S. Deser and R.I. Nepomechie, Anomalous propagation of gauge fields in conformally flat spaces, Phys. Lett. 132B (1983) 321 [INSPIRE].

[55] S. Deser and R.I. Nepomechie, Gauge invariance versus masslessness in de Sitter space, Annals Phys. 154 (1984) 396 [inSPIRE].

[56] A. Higuchi, Forbidden mass range for spin-2 field theory in de Sitter space-time, Nucl. Phys. B 282 (1987) 397 [INSPIRE].

[57] A. Higuchi, Symmetric tensor spherical harmonics on the $N$ sphere and their application to the de Sitter group $\mathrm{SO}(N, 1)$, J. Math. Phys. 28 (1987) 1553 [Erratum ibid. 43 (2002) 6385] [INSPIRE].

[58] A. Higuchi, Massive symmetric tensor Field in space-times with a positive cosmological constant, Nucl. Phys. B 325 (1989) 745 [INSPIRE].

[59] I.L. Buchbinder, D.M. Gitman, V.A. Krykhtin and V.D. Pershin, Equations of motion for massive spin-2 field coupled to gravity, Nucl. Phys. B 584 (2000) 615 [hep-th/9910188] [INSPIRE].

[60] I.L. Buchbinder, D.M. Gitman and V.D. Pershin, Causality of massive spin-2 field in external gravity, Phys. Lett. B 492 (2000) 161 [hep-th/0006144] [INSPIRE].

[61] S. Deser and A. Waldron, Gauge invariances and phases of massive higher spins in (A)dS, Phys. Rev. Lett. 87 (2001) 031601 [hep-th/0102166] [INSPIRE].

[62] S. Deser and A. Waldron, Partial masslessness of higher spins in (A)dS, Nucl. Phys. B 607 (2001) 577 [hep-th/0103198] [INSPIRE].

[63] S. Deser and A. Waldron, Stability of massive cosmological gravitons, Phys. Lett. B 508 (2001) 347 [hep-th/0103255] [INSPIRE].

[64] S. Deser and A. Waldron, Null propagation of partially massless higher spins in (A)dS and cosmological constant speculations, Phys. Lett. B 513 (2001) 137 [hep-th/0105181] [INSPIRE].

[65] Yu.M. Zinoviev, On massive high spin particles in AdS, hep-th/0108192 [INSPIRE].

[66] L. Dolan, C.R. Nappi and E. Witten, Conformal operators for partially massless states, JHEP 10 (2001) 016 [hep-th/0109096] [INSPIRE].

[67] S. Deser and A. Waldron, Conformal invariance of partially massless higher spins, Phys. Lett. B 603 (2004) 30 [hep-th/0408155] [INSPIRE].

[68] S. Deser, E. Joung and A. Waldron, Partial masslessness and conformal gravity, J. Phys. A 46 (2013) 214019 [arXiv:1208.1307] [INSPIRE].

[69] C. Brust and K. Hinterbichler, Partially massless higher-spin theory, JHEP 02 (2017) 086 [arXiv: 1610.08510$]$ [INSPIRE].

[70] M. Henneaux, S. Hörtner and A. Leonard, Higher spin conformal geometry in three dimensions and prepotentials for higher spin gauge fields, JHEP 01 (2016) 073 [arXiv: 1511.07389] [INSPIRE]. 
[71] M. Henneaux, V. Lekeu, A. Leonard, J. Matulich and S. Prohazka, Three-dimensional conformal geometry and prepotentials for four-dimensional fermionic higher-spin fields, JHEP 11 (2018) 156 [arXiv: 1810.04457] [INSPIRE].

[72] A. Iorio, L. O'Raifeartaigh, I. Sachs and C. Wiesendanger, Weyl gauging and conformal invariance, Nucl. Phys. B 495 (1997) 433 [hep-th/9607110] [INSPIRE].

[73] H. Linander and B.E.W. Nilsson, The non-linear coupled spin 2 - spin 3 Cotton equation in three dimensions, JHEP 07 (2016) 024 [arXiv: 1602.01682] [INSPIRE].

[74] T. Basile, R. Bonezzi and N. Boulanger, The Schouten tensor as a connection in the unfolding of $3 D$ conformal higher-spin fields, JHEP 04 (2017) 054 [arXiv:1701.08645] [INSPIRE].

[75] W. Siegel and S.J. Gates Jr., Superprojectors, Nucl. Phys. B 189 (1981) 295 [inSPIRE].

[76] S.J. Gates, M.T. Grisaru, M. Roček and W. Siegel, Superspace, or One Thousand and One Lessons in Supersymmetry, Front. Phys. 58 (1983) 1 [hep-th/0108200] [INSPIRE].

[77] R.E. Behrends and C. Fronsdal, Fermi decay of higher spin particles, Phys. Rev. 106 (1957) 345 [INSPIRE].

[78] C. Fronsdal, On the theory of higher spin fields, Nuovo Cim. 9 (1958) 416.

[79] J. Erdmenger and H. Osborn, Conformally covariant differential operators: Symmetric tensor fields, Class. Quant. Grav. 15 (1998) 273 [gr-qc/9708040] [INSPIRE].

[80] X. Bekaert and M. Grigoriev, Higher order singletons, partially massless fields and their boundary values in the ambient approach, Nucl. Phys. B 876 (2013) 667 [arXiv: 1305. 0162] [INSPIRE].

[81] M. Grigoriev and A. Hancharuk, On the structure of the conformal higher-spin wave operators, JHEP 12 (2018) 033 [arXiv: 1808.04320] [INSPIRE].

[82] P.S. Howe, J.M. Izquierdo, G. Papadopoulos and P.K. Townsend, New supergravities with central charges and Killing spinors in 2+1 dimensions, Nucl. Phys. B 467 (1996) 183 [hep-th/9505032] [INSPIRE].

[83] S.M. Kuzenko, U. Lindström and G. Tartaglino-Mazzucchelli, Off-shell supergravity-matter couplings in three dimensions, JHEP 03 (2011) 120 [arXiv:1101.4013] [INSPIRE].

[84] S.M. Kuzenko and G. Tartaglino-Mazzucchelli, Conformal supergravities as Chern-Simons theories revisited, JHEP 03 (2013) 113 [arXiv:1212.6852] [INSPIRE].

[85] S.M. Kuzenko, Prepotentials for $N=2$ conformal supergravity in three dimensions, JHEP 12 (2012) 021 [arXiv: 1209.3894] [INSPIRE].

[86] J. Hutomo and S.M. Kuzenko, Higher spin supermultiplets in three dimensions: (2,0) AdS supersymmetry, Phys. Lett. B 787 (2018) 175 [arXiv:1809.00802] [INSPIRE].

[87] D. Butter and J. Novak, Component reduction in $N=2$ supergravity: the vector, tensor and vector-tensor multiplets, JHEP 05 (2012) 115 [arXiv:1201.5431] [INSPIRE].

[88] D. Butter, S.M. Kuzenko and J. Novak, The linear multiplet and ectoplasm, JHEP 09 (2012) 131 [arXiv: 1205.6981] [INSPIRE].

[89] W. Siegel, Solution to constraints in Wess-Zumino supergravity formalism, Nucl. Phys. B 142 (1978) 301 [inSPIRE].

[90] B. Zumino, Supergravity and superspace, in Recent Developments in Gravitation - Cargèse 1978, M. Lévy and S. Deser (Eds.), Plenum Press, N.Y., U.S.A., (1979), pp. 405-459. 
[91] M.T. Grisaru and W. Siegel, Supergraphity (I). Background field formalism, Nucl. Phys. B 187 (1981) 149 [INSPIRE].

[92] M.T. Grisaru and W. Siegel, Supergraphity (II). Manifestly covariant rules and higher loop finiteness, Nucl. Phys. B 201 (1982) 292 [Erratum ibid. B 206 (1982) 496] [INSPIRE].

[93] S.M. Kuzenko, A.G. Sibiryakov and V.V. Postnikov, Massless gauge superfields of higher half-integer superspins, JETP Lett. 57 (1993) 534 [Pisma Zh. Eksp. Teor. Fiz. 57 (1993) 521] [INSPIRE].

[94] S.M. Kuzenko and A.G. Sibiryakov, Free massless higher superspin superfields on the anti-de Sitter superspace, Phys. Atom. Nucl. 57 (1994) 1257 [Yad. Fiz. 57 (1994) 1326] [arXiv: 1112.4612] [INSPIRE].

[95] S.M. Kuzenko and A.G. Sibiryakov, Massless gauge superfields of higher integer superspins, JETP Lett. 57 (1993) 539 [Pisma Zh. Eksp. Teor. Fiz. 57 (1993) 526] [INSPIRE].

[96] J. Hutomo and S.M. Kuzenko, The massless integer superspin multiplets revisited, JHEP 02 (2018) 137 [arXiv: 1711.11364] [INSPIRE].

[97] E.I. Buchbinder, J. Hutomo and S.M. Kuzenko, Higher spin supercurrents in anti-de Sitter space, JHEP 09 (2018) 027 [arXiv: 1805.08055] [INSPIRE].

[98] S.J. Gates Jr. and W. Siegel, (3/2, 1) superfield of O(2) supergravity, Nucl. Phys. B 164 (1980) 484 [INSPIRE].

[99] T.N. Bailey, M.G. Eastwood and A.R. Gover, Thomas's structure bundle for conformal, projective and related structures, Rocky Mt. J. Math. 24 (1994) 1191.

[100] A.R. Gover, Invariant theory and calculus for conformal geometries, Adv. Math. 163 (2001) 206.

[101] T.Y. Thomas, The Differential Invariants of Generalized Spaces, Cambridge University Press, (1934).

[102] A.R. Gover, A. Shaukat and A. Waldron, Tractors, mass and Weyl invariance, Nucl. Phys. B 812 (2009) 424 [arXiv:0810.2867] [INSPIRE].

[103] A.R. Gover, A. Shaukat and A. Waldron, Weyl invariance and the origins of mass, Phys. Lett. B 675 (2009) 93 [arXiv:0812.3364] [InSPIRE].

[104] R. Bonezzi, E. Latini and A. Waldron, Gravity, two times, tractors, Weyl invariance and six-dimensional quantum mechanics, Phys. Rev. D 82 (2010) 064037 [arXiv:1007.1724] [INSPIRE].

[105] M. Grigoriev and A. Waldron, Massive higher spins from BRST and tractors, Nucl. Phys. B 853 (2011) 291 [arXiv:1104.4994] [INSPIRE].

[106] E. Joung, M. Taronna and A. Waldron, A calculus for higher spin interactions, JHEP 07 (2013) 186 [arXiv: 1305.5809] [INSPIRE].

[107] D. Butter, F. Ciceri, B. de Wit and B. Sahoo, Construction of all $N=4$ conformal supergravities, Phys. Rev. Lett. 118 (2017) 081602 [arXiv: 1609.09083] [INSPIRE].

[108] I.A. Bandos, J. Lukierski and D.P. Sorokin, Superparticle models with tensorial central charges, Phys. Rev. D 61 (2000) 045002 [hep-th/9904109] [INSPIRE].

[109] M.A. Vasiliev, Conformal higher spin symmetries of 4 d massless supermultiplets and $\operatorname{osp}(L, 2 M)$ invariant equations in generalized (super)space, Phys. Rev. D 66 (2002) 066006 [hep-th/0106149] [INSPIRE]. 
[110] V.E. Didenko and M.A. Vasiliev, Free field dynamics in the generalized AdS (super)space, J. Math. Phys. 45 (2004) 197 [hep-th/0301054] [INSPIRE].

[111] O.A. Gelfond and M.A. Vasiliev, Higher rank conformal fields in the $\operatorname{Sp}(2 M)$ symmetric generalized space-time, Theor. Math. Phys. 145 (2005) 1400 [Teor. Mat. Fiz. 145 (2005) 35] [hep-th/0304020] [INSPIRE].

[112] M.A. Vasiliev and V.N. Zaikin, On $\operatorname{Sp}(2 M)$ invariant Green functions, Phys. Lett. B 587 (2004) 225 [hep-th/0312244] [INSPIRE].

[113] I. Bandos, P. Pasti, D. Sorokin and M. Tonin, Superfield theories in tensorial superspaces and the dynamics of higher spin fields, JHEP 11 (2004) 023 [hep-th/0407180] [INSPIRE].

[114] I. Bandos, X. Bekaert, J.A. de Azcarraga, D. Sorokin and M. Tsulaia, Dynamics of higher spin fields and tensorial space, JHEP 05 (2005) 031 [hep-th/0501113] [INSPIRE].

[115] E. Ivanov and J. Lukierski, Higher spins from nonlinear realizations of $\mathrm{OSp}(1 \mid 8)$, Phys. Lett. B 624 (2005) 304 [hep-th/0505216] [INSPIRE].

[116] O.A. Gelfond and M.A. Vasiliev, $\operatorname{Sp}(8)$ invariant higher spin theory, twistors and geometric BRST formulation of unfolded field equations, JHEP 12 (2009) 021 [arXiv:0901.2176] [INSPIRE].

[117] I.A. Bandos, J.A. de Azcarraga and C. Meliveo, Extended supersymmetry in massless conformal higher spin theory, Nucl. Phys. B 853 (2011) 760 [arXiv:1106.5199] [InSPIRE].

[118] I. Florakis, D. Sorokin and M. Tsulaia, Higher spins in hyperspace, JHEP 07 (2014) 105 [arXiv: 1401.1645$]$ [INSPIRE].

[119] I. Florakis, D. Sorokin and M. Tsulaia, Higher spins in hyper-superspace, Nucl. Phys. B 890 (2014) 279 [arXiv: 1408.6675] [INSPIRE].

[120] S. Fedoruk and J. Lukierski, New spinorial particle model in tensorial space-time and interacting higher spin fields, JHEP 02 (2013) 128 [arXiv:1210.1506] [INSPIRE].

[121] E. Skvortsov, D. Sorokin and M. Tsulaia, Correlation functions of $\operatorname{Sp}(2 n)$ invariant higher-spin systems, JHEP 07 (2016) 128 [arXiv: 1605.08498] [INSPIRE].

[122] M.A. Vasiliev, Holography, unfolding and higher-spin theory, J. Phys. A 46 (2013) 214013 [arXiv: 1203.5554] [INSPIRE].

[123] D. Sorokin and M. Tsulaia, Higher Spin Fields in Hyperspace. A Review, Universe 4 (2018) 7 [arXiv: 1710.08244] [INSPIRE]. 